\title{
One-Loop Diagrams in Lattice QCD with Wilson Fermions
}

\author{
Rogalyov R.N.* \\ Institute for High Energy Physics \\ Protvino, Moscow region, 142281, Russia
}

November 18, 2018

\begin{abstract}
A comprehensive number of integrals emerging in one-loop computations in a gauge perturbation theory on a lattice with Wilson fermions at $r=1$ is computed using the Burgio-Caracciolo-Pelissetto algorithm and the FORM package. An explicit analytical form of the recursion relations needed for such computations is presented.
\end{abstract}

*e-mail: rogalyov@th1.ihep.su 


\section{Introduction}

Perturbation theory provides a useful tool in the studies of the weak coupling limit of field theories on a lattice [1]. To simplify perturbative computations it is helpful to derive analytical expressions for the Feynman integrals. Typical integrals emerging in the one-loop approximation have the form

$$
\int_{B Z} \frac{d k}{(2 \pi)^{4}} \frac{\mathcal{P}\left(\hat{k}_{\mu_{0}},\left(k \widehat{-p_{1}}\right)_{\mu_{1}}, \ldots,\left(k-p_{1}-\ldots-p_{n}\right)_{\mu_{1}}\right)}{D_{\text {latt }}(k) D_{\text {latt }}\left(p_{1}-k\right) \ldots D_{\text {latt }}\left(p_{1}+\ldots+p_{n}-k\right)}
$$

where $\hat{p}_{\mu}=\frac{2}{a} \sin \left(\frac{p_{\mu} a}{2}\right), \quad D_{\text {latt }}(k)=\sum_{\mu=1}^{4} \hat{k}_{\mu}^{2}+m^{2}$ (in the boson case), symbol $\mathcal{P}$ in the numerator means "some polynomial of" and symbol $B Z$ indicates that integration is performed over the Brillouin zone, that is the domain $-\frac{\pi}{a}<k_{\mu}<\frac{\pi}{a}$.

Such integrals cannot be calculated analytically at finite values of $a$. In the limit $a \rightarrow 0$ they can be evaluated by the Kawai-Nakayama-Seo method [2].

The above integrand can be represented in the form

$$
I\left(k, \tilde{p}, m^{2} ; a\right)=I(k, 0,0 ; a)+\left(I\left(k, \tilde{p}, m^{2} ; a\right)-I(k, 0,0 ; a)\right)
$$

where $\tilde{p}$ is the set of all relevant external momenta, $m$ is the mass. $I\left(k, \tilde{p}, m^{2} ; a\right)-I(k, 0,0 ; a)$ has a smooth continuum limit $(p a \rightarrow 0$ and $m a \rightarrow 0)$ and involves no ultraviolet (UV) divergencies. It can be computed with some continuum regularization such as dimensional (DR) or with fictitious mass 1 (FMP).

Both $I(k, 0,0 ; a)$ and $\left(I\left(k, \tilde{p}, m^{2} ; a\right)-I(k, 0,0 ; a)\right)$ involve infrared (IR) divergencies.

Though these IR divergencies cancel each other, IR regularization is needed:

$$
I\left(k, \tilde{p}, m^{2} ; a\right)=\lim _{\mu_{R}^{2} \rightarrow 0} I\left(k, \tilde{p}, m^{2} ; a, \mu_{R}^{2}\right) .
$$

In this work, we use the infrared regularization introduced below in the formulas (44) for bosons and (52) for fermions. Thus the sought-for integral is represented as the sum of the integral over the Euclidean momentum space (which is readily calculated by well-known method) and the "zero-momentum" integral over the Brillouin zone. Calculation of the latter integrals forms the subject of the present study.

In recent years, considerable study was given to computations with rather complicated actions (see, for example [3] and [4]). In so doing, one is confronted with an integrand involving products of $\left(\hat{k}_{\mu}\right)^{n}$ at large values of $n$. Algorithms for computation of such integrals with both bosonic and fermionic denominators were proposed in [5] and [6].

An outline of this paper is as follows. In this work, the algorithm proposed in [5] is employed to obtain a comprehensive set of the integrals needed in computations of various matrix elements. For the reader's convenience, a detailed exposition of the Burgio-CaraccioloPelissetto (BCP) algorithm is given. In Section 1, we deal with the bosonic case. Making use of the BCP algorithm, we derive an explicit form of the recursion relations: formulas (24), (25), and (28). In Section 2 we describe computations in the fermionic case. In this Section,

\footnotetext{
${ }^{1}$ The regularization by fictitious mass is obtained by adding the term $\mu_{R}^{2}=2 \mu_{B}^{2}$ to the denominator of each boson or fermion propagator.
} 
we also begin with the exposition of the BCP algorithm and use it to find an explicit form of the recursion relations for the functions $B(p, q)$ and $J(p, q)$ related to the functions $\mathcal{F}_{\delta}(p, q)$ used in [5]. These relations are presented in the Appendices. As a matter of fact, they provide a computer program for a calculation of the general fermionic integrals (151). The results obtained in this way are discussed in the Conclusions.

\section{Boson Integrals}

We compute the bosonic 'zero-momentum' integrals of the type (we set $a=1$ )

$$
F\left(q, n_{1}, n_{2}, n_{3}, n_{4}\right)=\lim _{\delta \rightarrow 0} \int d k \frac{\left(\cos k_{1}\right)^{n_{1}}\left(\cos k_{2}\right)^{n_{2}}\left(\cos k_{3}\right)^{n_{3}}\left(\cos k_{4}\right)^{n_{4}}}{\Delta_{B}^{(q+\delta)}}
$$

where

$$
\Delta_{B}=4+\mu_{B}^{2}-\cos \left(k_{1}\right)-\cos \left(k_{2}\right)-\cos \left(k_{3}\right)-\cos \left(k_{4}\right)
$$

is the scalar boson propagator, $\mu_{B}$ is the infrared regulator mass, and $\delta$ is an infinitesimal parameter needed for an additional intermediate regularization. All integrals in one-loop calculations with the boson propagators can be reduced to the integrals of this type.

Since $F\left(q ; n_{1}, n_{2}, n_{3}, n_{4}\right)$ is symmetric in the arguments $n_{1}, n_{2}, n_{3}$, and $n_{4}$, we consider only the case

$$
n_{1} \geq n_{2} \geq n_{3} \geq n_{4}
$$

\subsection{Formulas of Reduction}

A computation of the massless 2 boson integrals over the Brillouin zone is based on the following algorithm:

$$
\begin{aligned}
& \text { if } \quad n_{4} \geq 2 \quad \text { then } \quad F\left(q, n_{1}, n_{2}, n_{3}, n_{4}\right)=F\left(q, n_{1}, n_{2}, n_{3}, n_{4}-2\right) \\
& -\frac{1}{q-1+\delta}\left(\left(n_{4}-1\right) F\left(q-1, n_{1}, n_{2}, n_{3}, n_{4}-1\right)-\left(n_{4}-2\right) F\left(q-1, n_{1}, n_{2}, n_{3}, n_{4}-3\right)\right) \text {, } \\
& \text { else if } \quad n_{3} \geq 2 \quad \text { then } \quad F\left(q, n_{1}, n_{2}, n_{3}, 0\right)=F\left(q, n_{1}, n_{2}, n_{3}-2,0\right) \\
& -\frac{1}{q-1+\delta}\left(\left(n_{3}-1\right) F\left(q-1, n_{1}, n_{2}, n_{3}-1,0\right)-\left(n_{3}-2\right) F\left(q-1, n_{1}, n_{2}, n_{3}-3,0\right)\right), \\
& \text { else if } \quad n_{2} \geq 2 \quad \text { then } \quad F\left(q, n_{1}, n_{2}, 0,0\right)=F\left(q, n_{1}, n_{2}-2,0,0\right) \\
& -\frac{1}{q-1+\delta}\left(\left(n_{2}-1\right) F\left(q-1, n_{1}, n_{2}-1,0,0\right)-\left(n_{2}-2\right) F\left(q-1, n_{1}, n_{2}-3,0,0\right)\right), \\
& \text { else if } \quad n_{1} \geq 2 \quad \text { then } \quad F\left(q, n_{1}, 0,0,0\right)=F\left(q, n_{1}-2,0,0,0\right) \\
& -\frac{1}{q-1+\delta}\left(\left(n_{1}-1\right) F\left(q-1, n_{1}-1,0,0,0\right)-\left(n_{1}-2\right) F\left(q-1, n_{1}-3,0,0,0\right)\right) \text {. } \\
& F\left(q, n_{1}, n_{2}, n_{3}, 1\right)=\left(4+\mu_{B}^{2}\right) F\left(q, n_{1}, n_{2}, n_{3}, 0\right)-F\left(q-1, n_{1}, n_{2}, n_{3}, 0\right) \\
& \text { - } F\left(q, n_{1}+1, n_{2}, n_{3}, 0\right)-F\left(q, n_{1}, n_{2}+1, n_{3}, 0\right)-F\left(q, n_{1}, n_{2}, n_{3}+1,0\right) \text {, }
\end{aligned}
$$

\footnotetext{
${ }^{2}$ Here $\mu_{B}$ is the regulator mass, thus we consider the massless limit at the end of computations.
} 


$$
\begin{aligned}
F\left(q, n_{1}, n_{2}, 1,0\right)= & \frac{1}{2}\left(\left(4+\mu_{B}^{2}\right) F\left(q, n_{1}, n_{2}, 0,0\right)-F\left(q-1, n_{1}, n_{2}, 0,0\right)\right. \\
& \left.-F\left(q, n_{1}+1, n_{2}, 0,0\right)-F\left(q, n_{1}, n_{2}+1,0,0\right)\right), \\
F\left(q, n_{1}, 1,0,0\right)= & \frac{1}{3}\left(\left(4+\mu_{B}^{2}\right) F\left(q, n_{1}, 0,0,0\right)-F\left(q-1, n_{1}, 0,0,0\right)\right. \\
& \left.-F\left(q, n_{1}+1,0,0,0\right)\right), \\
F(q, 1,0,0,0)= & \frac{1}{4}\left(\left(4+\mu_{B}^{2}\right) F(q, 0,0,0,0)-F(q-1,0,0,0,0)\right) .
\end{aligned}
$$

The above identities can be obtained using integration by parts [5]; order $O\left(\delta^{2}\right)$ terms should be omitted.

Thus we obtain an expression for each integral $F\left(q, n_{1}, n_{2}, n_{3}, n_{4}\right)$ in terms of the functions 3

$$
G_{\delta}\left(q, \mu_{B}^{2}\right)=\int \frac{d k}{(2 \pi)^{4}} \frac{1}{\left(\Delta_{B}\right)^{q+\delta}}
$$

it has the form

$$
\begin{aligned}
F\left(q, n_{1}, n_{2}, n_{3}, n_{4}\right) & =\sum_{r=q-n_{1}-n_{2}-n_{3}-n_{4}}^{q} a_{q r}\left(\delta, \mu_{B}^{2}, \tilde{n}\right) G_{\delta}\left(r, \mu_{B}^{2}\right) \\
& =\sum_{r=q-n_{1}-n_{2}-n_{3}-n_{4}}^{0} a_{q r}(\delta, 0, \tilde{n}) G_{\delta}(r, 0)+\sum_{r=1}^{q} a_{q r}\left(0, \mu_{B}^{2}, \tilde{n}\right) G\left(r, \mu_{B}^{2}\right)+\mathcal{O}\left(\mu_{B}^{2}\right),
\end{aligned}
$$

where $\tilde{n}$ is short-hand notation for $n_{1}, n_{2}, n_{3}, n_{4}$. In this sum, the terms with $r \leq 0$ and those with $r>0$ should be considered separately:

- The coefficients $a_{q r}\left(\delta, \mu_{B}^{2}, \tilde{n}\right)$ at $r \leq 0$ involve the pole $\frac{1}{\delta}$ :

$$
a_{q r}\left(\delta, \mu_{B}^{2}, \tilde{n}\right)=a_{q r}^{(s i n g)}\left(\mu_{B}^{2}, \tilde{n}\right) \frac{1}{\delta}+a_{q r}^{(r e g)}\left(\mu_{B}^{2}, \tilde{n}\right)+O(\delta),
$$

so that $G_{\delta}(r, 0)$ must be expanded to the order $\mathcal{O}(\delta)$. Since $G_{\delta}\left(r, \mu_{B}^{2}\right)$ has no infrared divergencies at $r \leq 0$ and the coefficients $a_{q r}\left(\delta, \mu_{B}^{2}, \tilde{n}\right)$ are polynomials in $\mu_{B}^{2}$, the values of $a_{q r}^{(s i n g)}$ and $a_{q r}^{(r e g)}$ should be evaluated at $\mu_{B}=0$.

- At $r>0$, the coefficients $a_{q r}\left(\delta, \mu_{B}^{2}, \tilde{n}\right)$ involve no poles in $\delta$ and, therefore, $\delta$ can be safely set to zero. However, at $r>0, G_{\delta}\left(r, \mu_{B}^{2}\right)$ involves infrared divergencies, so that the $\mu_{B}$ dependence of the coefficients $a_{q r}$ should be kept.

From these properties it follows that we should compute the quantities that appear in the right-hand sides of the formulas

$$
\begin{array}{rl}
\text { for } \quad r \leq 0, & G_{\delta}\left(r, \mu_{B}^{2}\right)=\mathcal{B}_{-r}+J(r) \delta+O\left(\delta^{2}\right)+\mathcal{O}\left(\mu_{B}^{2}\right), \\
r=1 & G\left(1, \mu_{B}^{2}\right)=J(1)+\mathcal{O}\left(\mu_{B}^{2}\right), \\
r=2 & G\left(2, \mu_{B}^{2}\right)=J(2)+\left(\ln \mu_{B}^{2}+C\right)+\mathcal{O}\left(\mu_{B}^{2}\right), \\
\text { for } \quad r>2, & G\left(r, \mu_{B}^{2}\right)=J(r)+D_{r 0}\left(\ln \mu_{B}^{2}+C\right)+\sum_{k=1}^{r-2} D_{r k} /\left(\mu_{B}\right)^{2 k}+\mathcal{O}\left(\mu_{B}^{2}\right),
\end{array}
$$

\footnotetext{
${ }^{3}$ In what follows, $G\left(q, \mu_{B}^{2}\right)=\lim _{\delta \rightarrow 0} G_{\delta}\left(q, \mu_{B}^{2}\right)$.
} 
where $C=0.577 \ldots$ is the Euler-Mascheroni constant, $D_{r n}$ and $J(r)$ are some constants to be determined and $\mathcal{B}_{r}$ are given by

$$
\mathcal{B}_{r}=\lim _{\mu_{B} \rightarrow 0} \int_{B Z} \frac{d k}{(2 \pi)^{4}} \Delta_{B}^{r}
$$

(note that $r>0$ ), some of them can be found in Appendix 1.

\subsection{Computation of the Divergent Part (Fictitious Mass Regularization)}

We consider the representation of $G_{\delta}\left(q, \mu_{B}^{2}\right)$ in terms of the modified Bessel function:

$$
G_{\delta}\left(q, \mu_{B}^{2}\right)=\int \frac{d k}{(2 \pi)^{4}} \frac{1}{\left(\Delta_{B}\right)^{q+\delta}}=\frac{1}{\Gamma(q+\delta)} \int_{0}^{\infty} t^{q-1+\delta} d t\left[e^{-4 t-\mu_{B}^{2} t} I_{0}^{4}(t)\right]
$$

and divide the domain of integration into two parts: $\int_{0}^{\infty}=\int_{0}^{1}+\int_{1}^{\infty}$. The integral over the segment $[0,1]$ converges. The divergent part arises from the latter integral and can be isolated by subtracting $q-1$ terms of the asymptotic expansion at $z \rightarrow \infty$ of the function

$$
\exp (-4 z) I_{0}^{4}(z) \simeq \frac{1}{(2 \pi z)^{2}}\left(1+\frac{b_{1}}{z}+\frac{b_{2}}{z^{2}}+\ldots\right)
$$

$b_{i}$ at $i \leq 20$ are given in Appendix 1. We isolate the divergent part $\bar{G}_{d i v}^{M}\left(q, \delta, \mu_{B}\right)$ as follows:

$$
\begin{aligned}
G_{\delta}\left(q, \mu_{B}^{2}\right)=\bar{G}_{d i v}^{M}\left(q, \delta, \mu_{B}\right)+\bar{J}_{\delta}(q)= & \frac{1}{\Gamma(q+\delta)}\left\{\int_{0}^{1} t^{q-1+\delta} d t\left[e^{-4 t-\mu_{B}^{2} t} I_{0}^{4}(t)\right]+\right. \\
& +\int_{1}^{\infty} t^{q-1+\delta} d t e^{-\mu_{B}^{2} t}\left[e^{-4 t} I_{0}^{4}(t)-\frac{1}{(2 \pi t)^{2}} \sum_{n=0}^{q-2} \frac{b_{n}}{t^{n}}\right] \\
& \left.+\int_{1}^{\infty} t^{q-1+\delta} d t \frac{1}{(2 \pi t)^{2}} e^{-\mu_{B}^{2} t} \sum_{n=0}^{q-2} \frac{b_{n}}{t^{n}}\right\}
\end{aligned}
$$

where the first and second lines are designated by $\bar{J}_{\delta}(q)$ and the third-by $\bar{G}_{d i v}^{M}\left(q, \delta, \mu_{B}\right)$ :

$$
\begin{aligned}
& \bar{G}_{d i v}^{M}\left(q, \delta, \mu_{B}\right)=\frac{1}{(2 \pi)^{2} \Gamma(q+\delta)} \sum_{n=0}^{q-2}\left(\int_{0}^{\infty}-\int_{0}^{1}\right) d t b_{n} t^{q-3-n+\delta} e^{-\mu_{B}^{2} t} \\
= & \frac{1}{(2 \pi)^{2} \Gamma(q)}\left[-b_{q-2} l_{C}+\sum_{k=1}^{q-2} b_{q-k-2}\left(\frac{\Gamma(k)}{\left(\mu_{B}^{2}\right)^{k}}-\frac{1}{k}\right)\right]+ \\
+ & \frac{\delta}{(2 \pi)^{2} \Gamma(q)}\left[b_{q-2}\left(\frac{1}{2} l_{C}^{2}+\frac{\pi^{2}}{12}+\psi(q) l_{C}\right)+\sum_{n=1}^{q-2} b_{q-2-n}\left(\frac{\Gamma(n)}{\left(\mu_{B}^{2}\right)^{n}}\left(\psi(n)-\psi(q)-l_{C}+C\right)+\frac{1}{n^{2}}\right)\right],
\end{aligned}
$$

where $\psi(n)=\sum_{k=1}^{n-1} \frac{1}{k}-C$ and $l_{C}=\left(\ln \mu_{B}^{2}+C\right)$.

In the case of purely boson integrals, $O(\delta)$ terms can be omitted; however, they are needed for a computation of the divergent part of the integrals (51) involving fermion denominators. 
We can also isolate the divergent part in the so called "minimal way"

$$
\begin{gathered}
G_{d i v}^{M}\left(q, \delta, \mu_{B}\right)=\frac{1}{(2 \pi)^{2} \Gamma(q)}\left\{\left[-b_{q-2} l_{C}+\sum_{k=1}^{q-2} b_{q-k-2} \frac{\Gamma(k)}{\left(\mu_{B}^{2}\right)^{k}}+\right.\right. \\
\left.+\delta\left[b_{q-2}\left(\frac{1}{2} l_{C}^{2}+\psi(q) l_{C}\right)+\sum_{n=1}^{q-2} b_{q-2-n} \frac{\Gamma(n)}{\left(\mu_{B}^{2}\right)^{n}}\left(\psi(n)-\psi(q)-l_{C}+C\right)\right]\right\} \\
\bar{G}_{d i v}^{M}\left(q, \delta, \mu_{B}\right)=G_{d i v}^{M}\left(q, \delta, \mu_{B}\right)-\frac{1}{(2 \pi)^{2} \Gamma(q)} \sum_{k=1}^{q-2} \frac{b_{q-k-2}}{k}+\frac{\delta}{(2 \pi)^{2} \Gamma(q)}\left[b_{q-2} \frac{\pi^{2}}{12}+\sum_{n=1}^{q-2} \frac{b_{q-2-n}}{n^{2}}\right] .
\end{gathered}
$$

Note that $J(q)$ that appears in (10) is connected with the quantity $\bar{J}(q)=\lim _{\delta \rightarrow 0} \bar{J}_{\delta}(q)$ (see (14)) by the relations

$$
G\left(q, \mu_{B}^{2}\right)=\lim _{\delta \rightarrow 0} G_{\delta}\left(q, \mu_{B}^{2}\right)=\bar{G}_{d i v}^{M}\left(q, 0, \mu_{B}^{2}\right)+\bar{J}(q)=G_{d i v}^{M}\left(q, 0, \mu_{B}^{2}\right)+J(q),
$$

so that

$$
\bar{J}(q)-J(q)=\frac{1}{(2 \pi)^{2} \Gamma(q)} \sum_{k=1}^{q-2} \frac{b_{q-k-2}}{k}
$$

and the coefficients $D_{r k}$ from the formula (10) are determined from the the equation (16):

$$
D_{r k}=\frac{b_{r-k-2} \Gamma(k)}{(2 \pi)^{2} \Gamma(r)} \text { at } 1 \leq k \leq r-2 ; \quad D_{r 0}=-\frac{b_{r-2}}{(2 \pi)^{2} \Gamma(r)} .
$$

\subsection{Computation of the Finite Parts (Fictitious Mass Regularization)}

Using the reduction formulas (6) and (17), we obtain expressions for the integrals $F\left(q ; n_{1}, n_{2}, n_{3}, n_{4}\right) \equiv$ $F(q, \tilde{n})$ in terms of the quantities $J(r), \mathcal{B}_{r}$, and $D_{r n}$ determined above (see formula (9)).

The next step is to use recursion relations making it possible to express $J(r)$ at $r \geq 4$ in terms of the basic boson constants $J(1), J(2)$, and $J(3)$ and at $r \leq 0$ in terms of $J(1), J(2)$, $J(3)$, and $J(0)$. The recursion relations are obtained by making use of the trivial identity

$$
\Delta_{B}-4-\mu_{B}^{2}+\sum_{\mu=1}^{4} \cos \left(k_{\mu}\right)=0 .
$$

Inserting this identity in the integrals

$$
F(q, 1,1,1,1)=\int d k \frac{\cos \left(k_{1}\right) \cos \left(k_{2}\right) \cos \left(k_{3}\right) \cos \left(k_{4}\right)}{\Delta_{B}^{(q+\delta)}}
$$

we arrive at

$$
\left(4+\mu_{B}^{2}\right) F(q, 1,1,1,1)=4 F(q, 2,1,1,1)+4 F(q-1,1,1,1,1),
$$

Now we express $F(q, 1,1,1,1)$ etc. in terms of the values $J(r)$ and thus obtain the sought for

relations between them. With these relations, $J(r)$ at $r \leq 0$ and $r \geq 4$ is readily expressed 
in terms of $J(1), J(2)$, and $J(3)$. We consider FMR with the finite part (16) defined in the "minimal" way. Given

$$
G_{d i v}^{M}\left(q, \mu_{B}^{2}\right)=\frac{1}{(2 \pi)^{2} \Gamma(q)}\left[b_{q-2} l_{C}+\sum_{k=1}^{q-2} b_{q-k-2} \frac{\Gamma(k)}{\left(\mu_{B}^{2}\right)^{k}}\right],
$$

we derive the recursion relations for $J(q) \quad(q>0)$ as follows:

$$
\begin{aligned}
J(q)= & \frac{1}{384(q-1)(q-2)^{2}(q-3)} \\
& \{16(q-2)(q-3)[12+25(q-2)(q-3)] J(q-1) \\
& +4(q-3)^{2}\left[-17-35(q-3)^{2}\right] J(q-2) \\
& +4\left[1+5(q-3)^{3}(q-4)-5(q-3)(q-4)^{2}\right] J(q-3) \\
& \left.-(q-4)^{4} J(q-4)\right\} \\
& +\frac{1}{(q-2)} D(q) \\
& -\frac{25}{24(q-1)(q-2)}(2 q-5) D(q-1) \\
& +\frac{1}{96(q-1)(q-2)^{2}}\left[17+105(q-3)^{2}\right] D(q-2) \\
& +\frac{5}{96(q-1)(q-2)^{2}(q-3)}\left[-1-4(q-3)^{2}(q-4)+2(q-4)^{2}\right] D(q-3) \\
& +\frac{5}{384(q-1)(q-2)^{2}(q-3)}(q-4)^{3} D(q-4) ;
\end{aligned}
$$

where $D(q)=\frac{b_{q-2}}{(q-1)}$; this being so, $D(q)$ satisfies the recurrent relation

$$
\begin{aligned}
D(q)= & \frac{1}{384(q-1)(q-2)^{2}(q-3)} \\
& \{16(q-2)(q-3)[12+25(q-2)(q-3)] D(q-1) \\
& +4(q-3)^{2}\left[-17-35(q-3)^{2}\right] D(q-2) \\
& +4\left[1+5(q-3)^{3}(q-4)-5(q-3)(q-4)^{2}\right] D(q-3) \\
& \left.-(q-4)^{4} D(q-4)\right\}
\end{aligned}
$$

with the initial conditions

$$
\begin{aligned}
& J(0)=J_{0} ; \\
& J(1)=2 Z_{0} ; \\
& J(2)=\frac{F_{0}}{(2 \pi)^{2}} ; \\
& J(3)=\frac{Z_{1}}{32}+\frac{1}{(2 \pi)^{2}} \frac{F_{0}}{4}-\frac{1}{(2 \pi)^{2}} \frac{13}{48}-\frac{1}{128} ;
\end{aligned}
$$


and

$$
D(1)=0 ; \quad D(2)=\frac{1}{(2 \pi)^{2}} ; \quad D(3)=\frac{1}{(2 \pi)^{2}} \frac{1}{4} .
$$

Recurrent relations for $J(q)$ at $q<0$ can be derived by the same token, they have the form

$$
\begin{aligned}
J(q) & =-\frac{1}{q^{4}}\left[-4\left(1+5(q+1) q+5(q+1)^{2} q^{2}\right) J(q+1)\right. \\
& +4(q+1)^{2}\left(17+35(q+1)^{2}\right) J(q+2) \\
& -16(q+2)(q+1)(25(q+2)(q+1)+12) J(q+3) \\
& +384(q+3)(q+2)^{2}(q+1) J(q+4) \\
& +\frac{2 q^{3}\left(3+5(q+3)^{2}(q+1)\right)}{(q+4)(q+3)(q+2)(q+1)} \mathcal{B}_{-q} \\
& +4 \frac{\left(-40 q^{6}-330 q^{5}-985 q^{4}-1376 q^{3}-1015 q^{2}-410 q-70\right)}{(q+4)(q+3)(q+2)(q+1)} \mathcal{B}_{-q-1} \\
& +8 \frac{(q+1)\left(105 q^{4}+788 q^{3}+1998 q^{2}+2052 q+788\right)}{(q+4)(q+3)(q+2)} \mathcal{B}_{-q-2} \\
& +32 \frac{(q+2)(q+1)\left(31(q+4)^{2}-81(q+3)^{2}\right)}{(q+4)(q+3)} \mathcal{B}_{-q-3} \\
& +768 \frac{(q+3)(q+2)(q+1)}{(q+4)} \mathcal{B}_{-q-4} \quad \text { for } \quad q \leq-3 .
\end{aligned}
$$

The values of $\mathcal{B}_{q}$ can be computed either directly by the formula (11) or with the use of the recurrent relations

$$
\begin{aligned}
\mathcal{B}_{q}=\frac{1}{q^{4}}[ & -384(q-1)(q-2)^{2}(q-3) \mathcal{B}_{q-4} \\
& +16(q-1)(q-2)(25(q-1)(q-2)+12) \mathcal{B}_{q-3} \\
& -4(q-1)^{2}\left(35(q-1)^{2}+17\right) \mathcal{B}_{q-2} \\
& \left.+4\left(q^{5}-(q-1)^{5}\right) \mathcal{B}_{q-1}\right]
\end{aligned}
$$

with the initial conditions

$$
\mathcal{B}_{0}=1 ; \quad \mathcal{B}_{1}=4 ; \quad \mathcal{B}_{2}=18 ; \quad \mathcal{B}_{3}=88
$$

The values of $J(-1), J(-2)$, and $J(-3)$ can be determined in the same way, the respective identities have the form

$$
\begin{aligned}
& J(-4)=-9 / 16(13 / 9-781 / 36 J(-3)+83 J(-2)-108 J(-1)+32 J(0)) ; \\
& J(-3)=16 / 27(-11 / 2+211 / 12 J(-2)-157 / 3 J(-1)+124 / 3 J(0)+16 J(1)) ; \\
& J(-2)=-3\left(-3 / 2+\frac{8}{(2 \pi)^{2}}-31 / 12 J(-1)+13 / 3 J(0)+4 J(1)\right) ; \\
& J(-1)=144\left(-1 / 36-\frac{13}{9(2 \pi)^{2}}+1 / 36 J(0)+4 / 3 J(2)-16 / 3 J(3)\right) ;
\end{aligned}
$$


The integrals (3) can also be expressed in therms of the quantities $l_{C}$, and

$$
\begin{aligned}
& Z_{0} \approx 0.154933390231060214084837208 \\
& Z_{1} \approx 0.107781313539874001343391550 \\
& F_{0} \approx F_{0}^{C}-\ln 2=4.369225233874758-\ln 2
\end{aligned}
$$

determined from the relations 4

$$
\begin{aligned}
& F(1,0,0,0,0)=2 Z_{0}+O\left(\mu_{B}^{2}\right) \\
& F(2,0,0,0,0)=-\frac{l_{C}}{(2 \pi)^{2}}+\frac{F_{0}}{(2 \pi)^{2}}+O\left(\mu_{B}^{2}\right) \\
& F(3,0,0,0,0)=\frac{1}{(2 \pi)^{2}}\left(\frac{1}{2 \mu_{B}^{2}}-\frac{l_{C}}{4}-\frac{13}{48}+\frac{F_{0}}{4}\right)-\frac{1}{128}+\frac{Z_{1}}{32}+O\left(\mu_{B}^{2}\right) .
\end{aligned}
$$

This being so, the initial conditions for the recurrent relations are given by the formula (26).

It should be noted that $J_{0}$ does not appear in the ultimate expressions for the integrals of the type (3), therefore, its numerical value is not needed.

\subsection{Dimensional Regularization}

First we introduce the quantity $\bar{J}(q ; \tilde{n})$ analogous to $\bar{J}_{\delta}(q)$ defined in (14):

$$
\begin{aligned}
\bar{J}(q ; \tilde{n}) & =\lim _{\mu_{B} \rightarrow 0} \lim _{\delta \rightarrow 0} \frac{1}{\Gamma(q+\delta)}\left\{\int_{0}^{1} t^{q-1+\delta} d t\left[e^{-\left(4+\mu_{B}^{2}\right) t} \mathcal{T}(\tilde{n})\right]+\right. \\
& \left.+\int_{1}^{\infty} t^{q-1+\delta} d t e^{-\mu_{B}^{2} t}\left[e^{-4 t} \mathcal{T}(\tilde{n})-\frac{1}{(2 \pi t)^{2}} \sum_{k=0}^{q-2} \frac{b_{k}(\tilde{n})}{t^{k}}\right]\right\}
\end{aligned}
$$

where

$$
\mathcal{T}(\tilde{n})=\left[\left(\frac{\partial}{\partial t}\right)^{n_{1}} I_{0}(t)\right]\left[\left(\frac{\partial}{\partial t}\right)^{n_{2}} I_{0}(t)\right]\left[\left(\frac{\partial}{\partial t}\right)^{n_{3}} I_{0}(t)\right]\left[\left(\frac{\partial}{\partial t}\right)^{n_{4}} I_{0}(t)\right] .
$$

It represents the finite part of the general boson integral (3) provided that the divergent part is defined by the formula similar to (15). We omit here $O(\delta)$ terms because they are only needed for the calculation of fermion integrals in the fictitious mass regularization. Thus we set $\delta=0$. Then it should be noted that

$$
\begin{aligned}
\bar{J}(q ; \tilde{n}) & =\lim _{\epsilon \rightarrow 0} \frac{1}{\Gamma(q)}\left\{\int_{0}^{1} t^{q-1} d t\left[e^{-(4-2 \epsilon) t} I_{0}^{-2 \epsilon}(t) \mathcal{T}(\tilde{n})\right]+\right. \\
& +\int_{1}^{\infty} t^{q-1} d t\left[e^{-(4-2 \epsilon) t} I_{0}^{-2 \epsilon}(t) \mathcal{T}(\tilde{n})-\frac{1}{(2 \pi t)^{2-\epsilon}} \sum_{k=0}^{q-2} \frac{\tilde{b}_{k}(\tilde{n})}{t^{k}}\right],
\end{aligned}
$$

where

$$
\tilde{b}_{k}(\tilde{n})=b_{k}(\tilde{n})-2 \epsilon d_{k}(\tilde{n}),
$$

where $b_{k}(\tilde{n})$ are the coefficients of the asymptotic expansion at $t \rightarrow \infty$

$$
(2 \pi t)^{2} e^{-4 t} \mathcal{T}(\tilde{n}) \simeq \sum_{k=1}^{\infty} \frac{b_{k}(\tilde{n})}{t^{k}}
$$

\footnotetext{
${ }^{4}$ In the review [1], the constant $F_{0}^{C} \approx 4.369225233874758$ is designated by $F_{0}$.
} 
and $d_{k}(\tilde{n})$ are the coefficients of the asymptotic expansion

$$
(2 \pi t)^{2} e^{-4 t} \mathcal{T}(\tilde{n}) \ln \left[e^{-t} I_{0}(t) \sqrt{2 \pi t}\right] \simeq \sum_{n=1}^{\infty} \frac{d_{n}(\tilde{n})}{t^{n}}
$$

Now we define the general boson integral (3) in the dimensional regularization by the formula

$$
F(q ; \tilde{n})=\bar{J}(q ; \tilde{n})+F_{d i v}^{D R}(q ; \tilde{n})
$$

where

$$
F_{d i v}^{D R}(q ; \tilde{n})=\int_{1}^{\infty} t^{q-1} d t \frac{1}{(2 \pi t)^{2-\epsilon}} \sum_{k=0}^{q-2} \frac{\tilde{b}_{k}(\tilde{n})}{t^{k}}
$$

and the dimensional regularization implies that

$$
\int_{1}^{\infty} d t t^{n+\epsilon}=0 \quad \text { at } n \neq-1, \quad \int_{1}^{\infty} \frac{d t}{t^{1-\epsilon}}=-\frac{1}{\epsilon} .
$$

This being so,

$$
F_{\text {div }}^{D R}(q ; \tilde{n})=\frac{1}{(2 \pi)^{2}} \frac{1}{\Gamma(q)}\left\{-\frac{1}{\epsilon} b_{q-2}(\tilde{n})-\ln (2 \pi) b_{q-2}(\tilde{n})+2 d_{q-2}(\tilde{n})\right\} .
$$

Now we isolate the "canonical" divergent part in the dimensional regularization; that is,

$$
\bar{F}_{d i v}^{D R}\left(q ; \tilde{n}, \mu^{2}\right)=-\frac{1}{(2 \pi)^{2}} \frac{1}{\Gamma(q)}\left[\frac{1}{\epsilon}-C+\ln \left(\frac{4 \pi}{\mu^{2}}\right)\right] b_{q-2}(\tilde{n}) .
$$

where $\mu$ is the parameter of dimensional regularizations; the "canonical" divergent part is needed to compensate for the infrared divergent part in the respective continuum integral with nonvanishing external momenta. We see that

$$
\begin{aligned}
F_{d i v}^{D R}(q ; \tilde{n},) & =\bar{F}_{d i v}^{D R}\left(q ; \tilde{n}, \mu^{2}\right) \\
& +\frac{1}{(2 \pi)^{2}} \frac{1}{\Gamma(q)}\left[\left(-C+\ln \frac{2}{\mu^{2}}\right) b_{q-2}(\tilde{n})+2 d_{q-2}(\tilde{n})\right] .
\end{aligned}
$$

The respective finite parts can be determined by the formula

$$
F^{D R}(q ; \tilde{n})=\bar{J}(q ; \tilde{n})+F_{d i v}^{D R}(q ; \tilde{n})=\bar{J}^{D R}(q ; \tilde{n})+\bar{F}_{d i v}^{D R}(q ; \tilde{n}),
$$

Now we express $\bar{J}^{D R}(q ; \tilde{n})$ in terms of the quantity $J(q ; \tilde{n})$ which can be calculated in the fictitious mass regularization by the method described below. First we note that $\bar{J}(q ; \tilde{n})$ is connected with $\bar{J}^{D R}(q ; \tilde{n})$ by the relation

$$
\bar{J}^{D R}(q ; \tilde{n})=\bar{J}(q ; \tilde{n})+\frac{1}{(2 \pi)^{2}} \frac{1}{\Gamma(q)}\left[(-C+\ln 2) b_{q-2}(\tilde{n})+2 d_{q-2}(\tilde{n})\right] .
$$

The relation between $\bar{J}(q ; \tilde{n})$ and $J(q ; \tilde{n})$ is derived from the formula

$$
F^{F M R}(q ; \tilde{n})=\bar{J}(q ; \tilde{n})+\bar{F}_{d i v}^{M}(q ; \tilde{n})=J(q ; \tilde{n})+F_{d i v}^{M}(q ; \tilde{n}),
$$

\footnotetext{
${ }^{5}$ In this subsection it is considered that intergation in (3) is performed over the $4-2 \epsilon$ dimensional space; the integral under consideration should be multiplied by $\mu^{2 \epsilon}$
} 
(it is the definition of $J(q ; \tilde{n}))$. From the formula analogous to (17) it follows that

$$
\begin{aligned}
\bar{J}(q ; \tilde{n}) & =J(q ; \tilde{n})+\left(F_{d i v}^{M}(q ; \tilde{n})-\bar{F}_{d i v}^{M}(q ; \tilde{n})\right) \\
& =J(q ; \tilde{n})+\frac{1}{(2 \pi)^{2} \Gamma(q)} \sum_{k=1}^{q-2} \frac{b_{q-k-2}(\tilde{n})}{k},
\end{aligned}
$$

where $\bar{F}_{d i v}^{M}(q ; \tilde{n})$ and $F_{d i v}^{M}(q ; \tilde{n})$ are natural analogs of the quantities $\bar{G}_{d i v}^{M}(q)$ and $G_{d i v}^{M}(q)$ introduced above. Combining formula (49) with (45) and (46), we arrive at

$$
\begin{aligned}
F^{D R}(q ; \tilde{n})= & \bar{F}_{d i v}^{D R}(q ; \tilde{n} ; \mu)+\bar{J}^{D R}(q ; \tilde{n} ; \mu), \text { where } \\
\bar{F}_{d i v}^{D R}(q ; \tilde{n}, \mu)= & -\frac{1}{(2 \pi)^{2}} \frac{1}{\Gamma(q)}\left[\frac{1}{\epsilon}-C+\ln \left(\frac{4 \pi}{\mu^{2}}\right)\right] b_{q-2}(\tilde{n}), \\
\bar{J}^{D R}(q ; \tilde{n} ; \mu)= & J(q ; \tilde{n})+\frac{1}{(2 \pi)^{2} \Gamma(q)} \sum_{k=1}^{q-2} \frac{b_{q-k-2}(\tilde{n})}{k} \\
& +\frac{1}{(2 \pi)^{2}} \frac{1}{\Gamma(q)}\left[(-C+\ln 2) b_{q-2}(\tilde{n})+2 d_{q-2}(\tilde{n})\right],
\end{aligned}
$$

where $J(q ; \tilde{n})$ can be calculated as follows: using the relations (66) and (77), $F(q, \tilde{n})$ is transformed to a linear combination of the quantities $G_{\delta}\left(r, \mu_{B}^{2}\right)$ (9) and the substitutions (10) are employed. In the resulting expression, $\mu_{B}^{-1}$ and $l_{C}$ are formally set equal to zero, all that remains represents the sought-for $J(q ; \tilde{n})$.

It should be noticed that $\frac{1}{\epsilon}$ appears in $F^{D R}(q ; \tilde{n})$ only in the combination $\frac{1}{\epsilon}-\ln 2-F_{0}+$ $\ln \left(\frac{4 \pi}{\mu^{2}}\right)$ (the Euler-Mascheroni constant $C$ cancels in the total expression).

\section{Fermion Integrals}

Here we consider the integrals (remember that $a=1$ )

$$
F(p, q ; \tilde{n})=\lim _{\delta \rightarrow 0} \int \frac{d^{4} k}{(2 \pi)^{4}} \frac{\cos ^{n_{1}}\left(k_{1}\right) \cos ^{n_{2}}\left(k_{2}\right) \cos ^{n_{3}}\left(k_{3}\right) \cos ^{n_{4}}\left(k_{4}\right)}{\Delta_{B}^{q} \Delta_{F}^{p+\delta}}
$$

where $p>0, \delta$ is a regularization parameter, and

$$
\Delta_{F}=10-4 \sum_{\mu=1}^{4} \cos \left(k_{\mu}\right)+\sum_{1 \leq \mu<\nu \leq 4} \cos \left(k_{\mu}\right) \cos \left(k_{\nu}\right)+\mu_{B}^{2}
$$

is the denominator of the fermionic propagator. Making use of the recursion relations

$$
\begin{aligned}
F(p, q, \ldots, l, \ldots)=F(p, q, \ldots, l-2, \ldots) \\
\quad+\mu_{B}^{2}(F(p, q, \ldots, l-1, \ldots)-F(p, q, \ldots, l-3, \ldots)) \\
\quad-(F(p, q-1, \ldots, l-1, \ldots)-F(p, q-1, \ldots, l-3, \ldots)) \\
\quad-\frac{q}{p-1+\delta}(F(p-1, q+1, \ldots, l-1, \ldots)-F(p-1, q+1, \ldots, l-3, \ldots)) \\
\quad-\frac{1}{p-1+\delta}((l-2) F(p-1, q, \ldots, l-2, \ldots)-(l-3) F(p-1, q, \ldots, l-4, \ldots)) ;
\end{aligned}
$$




$$
\begin{aligned}
& F(p, q ; n 1, n 2, n 3,2)=F(p, q-2, n 1, n 2, n 3,0)-2 \mu_{B}^{2} F(p, q-1, n 1, n 2, n 3,0) \\
& -2 F(p-1, q, n 1, n 2, n 3,0)+\left(4+2 \mu_{B}^{2}+\mu_{B}^{4}\right) F(p, q, n 1, n 2, n 3,0) \\
& -F(p, q, n 1+2, n 2, n 3,0)-F(p, q, n 1, n 2+2, n 3,0) \\
& -F(p, q, n 1, n 2, n 3+2,0) \text {, } \\
& F(p, q, n 1, n 2, n 3,1)=\left(\mu_{B}^{2}+4\right) F(p, q, n 1, n 2, n 3,0) \\
& -F(p, q-1, n 1, n 2, n 3,0)-F(p, q, n 1+1, n 2, n 3,0) \\
& -F(p, q, n 1, n 2+1, n 3,0)-F(p, q, n 1, n 2, n 3+1,0) \\
& F(p, q ; n 1, n 2,2,0)=\frac{1}{2}\left(F(p, q-2, n 1, n 2,0,0)-2 \mu_{B}^{2} F(p, q-1 ; n 1, n 2,0,0)\right. \\
& -2 F(p-1, q ; n 1, n 2,0,0)+\left(4+2 \mu_{B}^{2}+\mu_{B}^{4}\right) F(p, q, n 1, n 2,0,0) \\
& -F(p, q ; n 1+2, n 2,0,0)-F(p, q ; n 1, n 2+2,0,0)) \text {; } \\
& F(p, q ; n 1, n 2,1,0)=\frac{1}{2}\left(\left(\mu_{B}^{2}+4\right) F(p, q ; n 1, n 2,0,0)-F(p, q-1 ; n 1, n 2,0,0)\right. \\
& -F(p, q ; n 1+1, n 2,0,0)-F(p, q ; n 1, n 2+1,0,0)) \text {; } \\
& F(p, q ; n 1,2,0,0)=\frac{1}{3}\left(F(p, q-2 ; n 1,0,0,0)-2 \mu_{B}^{2} F(p, q-1 ; n 1,0,0,0)\right. \\
& -2 F(p-1, q ; n 1,0,0,0)+\left(4+2 \mu_{B}^{2}+\mu_{B}^{4}\right) F(p, q ; n 1,0,0,0) \\
& -F(p, q ; n 1+2,0,0,0)) \text {; } \\
& F(p, q ; n 1,1,0,0)=\frac{1}{3}\left(\left(\mu_{B}^{2}+4\right) F(p, q ; n 1,0,0,0)-F(p, q-1 ; n 1,0,0,0)\right. \\
& -F(p, q ; n 1+1,0,0,0)) \text {; } \\
& F(p, q ; 2,0,0,0)=\frac{1}{4}\left(F(p, q-2 ; 0,0,0,0)-2 \mu_{B}^{2} F(p, q-1 ; 0,0,0,0)\right. \\
& \left.-2 F(p-1, q, 0,0,0,0)+\left(4+2 \mu_{B}^{2}+\mu_{B}^{4}\right) F(p, q ; 0,0,0,0)\right) \text {; } \\
& F(p, q ; 1,0,0,0)=\frac{1}{4}\left(\left(\mu_{B}^{2}+4\right) F(p, q ; 0,0,0,0)-F(p, q-1 ; 0,0,0,0)\right) ;
\end{aligned}
$$


To compute the basic integrals $G_{\delta}\left(p, q ; \mu_{B}^{2}\right)$, we consider the cases $p>0$ and $p \leq 0$ separately. At $p>0$, only zeroth order of the expansion of $G_{\delta}(p, q)$ in a power series in $\delta$ gives a nonvanishing contribution, whereas at $p \leq 0$ one should also keep the term linear in $\delta$.

It is convenient 6 to represent $G_{\delta}(p, q)$ in the form

$$
\begin{aligned}
& G_{\delta}(p, q)=D\left(p, q ; \mu_{B}^{2}\right)+B(p, q)+\delta\left(L\left(p, q ; \mu_{B}^{2}\right)+J(p, q)\right)+O\left(\delta^{2}\right), \quad p \leq 0 ; \\
& G_{\delta}(p, q)=D\left(p, q ; \mu_{B}^{2}\right)+J(p, q)+O(\delta), \quad p>0 .
\end{aligned}
$$

where the quantities $B(p, q), D\left(p, q ; \mu_{B}^{2}\right), L\left(p, q ; \mu_{B}^{2}\right)$, and $J(p, q)$ are defined as follows:

$D\left(p, q ; \mu_{B}^{2}\right)+\delta L\left(p, q ; \mu_{B}^{2}\right)$ is the divergent part of $G_{\delta}\left(p, q ; \mu_{B}^{2}\right)$ at $p \leq 0$ (up to terms $O\left(\delta^{2}\right)$ ), $D\left(p, q ; \mu_{B}^{2}\right)$ is the divergent part of $G_{\delta}\left(p, q ; \mu_{B}^{2}\right)$ at $p>0$ (up to terms $O(\delta)$ ), $B(p, q)+\delta J(p, q)$ and $J(p, q)$ are the respective finite part: 7 .

The finite and divergent parts are unambiguously fixed by the requirement that $D\left(p, q ; \mu_{B}^{2}\right)$ and $L\left(p, q ; \mu_{B}^{2}\right)$ can be represented in the form

$$
\begin{aligned}
D\left(p, q ; \mu_{B}^{2}\right)= & D_{0}(p, q)\left(\ln \mu_{B}^{2}+C\right)+\sum_{r=1}^{p+q-2} \frac{D_{r}(p, q)}{\left(\mu_{B}^{2}\right)^{r}} \\
L\left(p, q ; \mu_{B}^{2}\right)= & \frac{1}{2} L_{0}^{(2)}(p, q)\left(\ln \mu_{B}^{2}+C\right)^{2}+L_{0}^{(1)}(p, q)\left(\ln \mu_{B}^{2}+C\right) \\
& +\sum_{r=1}^{p+q-2} \frac{L_{r}^{(2)}(p, q)\left(\ln \mu_{B}^{2}+C\right)}{\left(\mu_{B}^{2}\right)^{r}}+\sum_{r=1}^{p+q-2} \frac{L_{r}^{(1)}(p, q)}{\left(\mu_{B}^{2}\right)^{r}} .
\end{aligned}
$$

In the domain $p \leq 0$ we also use the quantities

$$
\mathcal{B}\left(p, q ; \mu_{B}^{2}\right)=B(p, q)+D\left(p, q ; \mu_{B}^{2}\right) \quad \text { and } \quad \mathcal{J}\left(p, q ; \mu_{B}^{2}\right)=L\left(p, q ; \mu_{B}^{2}\right)+J(p, q) .
$$

At $p>0$,

$$
\mathcal{B}\left(p, q ; \mu_{B}^{2}\right)=B(p, q)=0 \quad \text { and } \quad \mathcal{J}\left(p, q ; \mu_{B}^{2}\right)=D\left(p, q ; \mu_{B}^{2}\right)+J(p, q) .
$$

Note that, at $q<2-p, \quad D\left(p, q, \mu_{B}^{2}\right)=L\left(p, q, \mu_{B}^{2}\right)=0$, thus $\mathcal{B}\left(p, q ; \mu_{B}^{2}\right)=B(p, q)$ and $\mathcal{J}\left(p, q ; \mu_{B}^{2}\right)=J(p, q)$ and one can use both designations.

\subsection{Divergent Part in the Fictitious Mass Regularization}

First we note that (symbol DP means 'divergent part of')

$$
\begin{array}{ll}
p \leq 0, q \geq 2-p & D\left(p, q ; \mu_{B}^{2}\right)=\mathcal{D P} \int \frac{d k}{(2 \pi)^{4}} \frac{\Delta_{F}^{-p}}{\Delta_{B}^{q}}, \\
p=0, q \geq 2 & L\left(0, q ; \mu_{B}^{2}\right)=\left.\frac{d}{d \delta}\right|_{\delta=0} \mathcal{D P} \int \frac{d k}{(2 \pi)^{4}} \frac{1}{\Delta_{B}^{q+\delta}} \\
+\sum_{l=1}^{q-2} \frac{(-1)^{l}}{l} \mathcal{D P} \int \frac{d k}{(2 \pi)^{4}} \frac{\Delta^{l}}{\Delta_{B}^{l+q}}, \\
p>0, q \geq 2-p & D\left(p, q ; \mu_{B}^{2}\right)=\sum_{l=0}^{p+q-2} \frac{(-1)^{l}(p+l-1) !}{l !(p-1) !} \mathcal{D P} \int \frac{d k}{(2 \pi)^{4}} \frac{\Delta^{l}}{\Delta_{B}^{p+q+l}},
\end{array}
$$

\footnotetext{
${ }^{6}$ It should be noted that the functions $G_{\delta}(p, q)$ are related to the functions $\mathcal{F}_{\delta}(p, q)$ used in $[5$ by the formulas $\mathcal{F}_{\delta}(p, q)=2^{-p-q} G_{\delta}(p, q)$.

${ }^{7}$ Note that $J(p, q)$ designates the finite part in the order $O(1)$ at $p>0$ and in the order $O(\delta$ at $p \leq 0$
} 
The divergent parts of $\delta$-independent integrals in formulas (62) can be calculated as follows. First one employs the recursion relations (6) and (7) for boson integrals to transform the integrand to a linear combination of the basic boson integrals (12) and then evaluates the divergent part of each integral by the formula

$$
\mathcal{D P} \int \frac{d k}{(2 \pi)^{4}} \frac{1}{\Delta_{B}^{q}}=\frac{1}{(2 \pi)^{2} \Gamma(q)}\left[-b_{q-2} l_{C}+\sum_{k=1}^{q-2} b_{q-k-2} \frac{\Gamma(k)}{\left(\mu_{B}^{2}\right)^{k}}\right]
$$

(see derivation of the formula (16)), according to the MS prescription in the FMR. The $\delta$ dependent divergent parts that appears in formula (62) can determined by the same token, however, with the use of the formula

$$
\begin{aligned}
& \left.\frac{d}{d \delta}\right|_{\delta=0} \mathcal{D P} \int \frac{d k}{(2 \pi)^{4}} \frac{1}{\Delta_{B}^{q+\delta}}= \\
& =\frac{1}{(2 \pi)^{2} \Gamma(q)}\left[b_{q-2}\left(\frac{1}{2} l_{C}^{2}+\psi(q) l_{C}\right)+\sum_{n=1}^{q-2} b_{q-2-n} \frac{\Gamma(n)}{\left(\mu_{B}^{2}\right)^{n}}\left(\psi(n)-\psi(q)-l_{C}+C\right)\right] ;
\end{aligned}
$$

(see (16) $)$. The divergent parts at $p \leq 0$ can be obtained by the recursion relations, see below.

The divergent parts $D\left(p, q ; \mu_{B}^{2}\right)$ and $L\left(p, q ; \mu_{B}^{2}\right)$ introduced in the formula (58) are presented in Appendix 2 at $p+q \leq 8$; at other values it can be readily calculated by the above formulas.

\subsection{Finite Parts}

Given the divergent part, we use the recurrent relations (53) and (54) to express any integral of the type (51) in terms of the functions $B(p, q)$ and $J(p, q)$, which, in their turn, can be found by making use of the two types of the recursion relations [5].

The relations of the first type (the so called $T$-identities) can be obtained by inserting the expression $\Delta_{B}-4-\mu_{B}^{2}+\sum_{\mu=1}^{4} \cos \left(k_{\mu}\right)$ which is identically equals zero, in the integrand

$$
F(q, 1,1,1,1)=\int d k \frac{\cos \left(k_{1}\right) \cos \left(k_{2}\right) \cos \left(k_{3}\right) \cos \left(k_{4}\right)}{\Delta_{F}^{(p+\delta)} \Delta_{B}^{q}}
$$

The relations of the second type ( $S$-identities) can be obtained by inserting

$$
10-4 \sum_{\mu=1}^{4} \cos \left(k_{\mu}\right)+\sum_{1 \leq \mu<\nu \leq 4} \cos \left(k_{\mu}\right) \cos \left(k_{\nu}\right)+\mu_{B}^{2}-\Delta_{F},
$$

which is also identically equals zero, in the same integrand. In so doing, we arrive at

$$
\begin{aligned}
\left.\left(4+\mu_{B}^{2}\right) F(p, q, 1,1,1,1)-F(p, q-1,1,1,1,1)-4 F(p, q, 2,1,1,1)\right) & =0, \\
\left(4+2 \mu_{B}^{2}+\mu_{B}^{4}\right) F(p+1, q, 1,1,1,1)-2 \mu_{B}^{2} F(p+1, q-1,1,1,1,1) & \\
-4 F(p+1, q, 3,1,1,1)+F(p+1, q-2,1,1,1,1)-2 F(p, q, 1,1,1,1) & =0 .
\end{aligned}
$$

Then we express the functions $F(p, q ; \tilde{n})$ emerging here in terms of $B(p, q)$ and $J(p, q)$ and obtain the sought for identities. Due to the terms singular in $\delta$, the cases $p=1,2,3$ should be considered separately and, in the case $p \leq 0$, one should remember that only the order $O(\delta)$ part is nontrivial. 
In what follows, we indicate how to use the explicit form of the derived recurrent relations for the $\mathcal{B}$ and $\mathcal{J}$ functions presented in Appendices 3-11 in order to compute $8(p, q)$ and $J(p, q)$ at $p<9$ and any values of $q$. Note that some of the relations deal with $B(p, q)$ and $J(p, q)$, whereas the other-with $\mathcal{B}(p, q)$ and $\mathcal{J}(p, q)$. In the appendices, $B(p, q)$ and $J(p, q)$ are designated by $\mathrm{B}(\mathrm{p}, \mathrm{q})$ and $\mathrm{J}(\mathrm{p}, \mathrm{q})$, respectively, whereas $\mathcal{B}(p, q)$ and $\mathcal{J}(p, q)$ are designated by $\operatorname{BB}(p, q)$ and $J J(p, q)$.

Both the divergent and the finite parts as well as the recurrent relations were obtained using the FORM [7] and (partially) REDUCE [8] packages.

\subsection{1 $\mathcal{B}$ functions}

First we compute the $\mathcal{B}$ functions defined by the formula (60) (see also (58)).

- $\mathcal{B}$ functions at $p=0, q>0$

$$
\mathcal{B}\left(0, q, \mu_{B}^{2}\right)=G_{d i v}^{M}\left(q, \mu_{B}^{2}\right)+J(q),
$$

where $G_{d i v}^{M}(q)$ is defined by the equation (23) $\left(G_{d i v}^{M}(q) \neq 0\right.$ only at $\left.q \geq 2\right)$

and $J(q)$ at $q \geq 4$-by the recurrent relations 9 (24). The initial conditions are provided by $J(q)$ (and, therefore, $\left.\mathcal{B}\left(0, q, \mu_{B}^{2}\right)\right)$ at $1 \leq q \leq 3$ (see the formulas (26), (27)), and (32) ).

- $\mathcal{B}$ functions at $p=0, q \leq 0$

$$
\mathcal{B}\left(0, q, \mu_{B}^{2}\right)=\mathcal{B}_{-q}
$$

where $\mathcal{B}_{-q}$ are defined in the formula (11) and can also be determined from the recurrent relations (29) with the initial conditions (30) (some values are given in Appendix 1).

- $\mathcal{B}$ functions at $p \leq-1, q \leq 0$

In this domain, $\mathcal{B}\left(p, q ; \mu_{B}^{2}\right)$ functions involve no divergencies: $\mathcal{B}\left(p, q ; \mu_{B}^{2}\right)=B(p, q)=\mathcal{B}(p, q ; 0)$. Provided that $B(0, q)=\mathcal{B}_{-q}$ at $q \leq 0$ are known, $B(p, q)$ at $p<0, q \leq 0$ can be determined by the recurrent relations for the domain $p<0, q \geq 4$ (see below), however, with $\mu_{B}=0$, and, therefore, with $B(p, q)$ instead of $\mathcal{B}(p, q)$.

- $\mathcal{B}$ functions over the strip $p \leq-1,1 \leq q \leq 3$

In this domain, $\mathcal{B}(p, q)=B(p, q)$ unless $(p, q)=(-1,3)$.

$\mathcal{B}$ functions at $-2 \leq p \leq-1,1 \leq q \leq 3$ can be calculated by the formula

$$
\mathcal{B}(p, q)=\int d k \frac{\Delta_{F}^{-p}\left(k, \mu_{B}^{2}\right)}{\Delta_{B}^{q}\left(k, \mu_{B}^{2}\right)},
$$

\footnotetext{
${ }^{8}$ For more detail, see http://www.lattice.itep.ru/ pbaivid/lattpt/ or contact me via e-mail

${ }^{9}$ Note that $J(q)$ has nothing to do with $J(p, q)$ or $J(0, q)$.
} 
the result is as follows:

$$
\begin{aligned}
& B(-1,1)=1+12 Z_{1} \\
& B(-1,2)=4 Z_{0} \\
& B(-1,3)=\frac{1}{(2 \pi)^{2}}\left(\frac{1}{2}+F_{0}\right)+\frac{1}{2} Z_{0}, \quad\left[\mathcal{B}(-1,3)=B(-1,3)-\frac{l_{C}}{(2 \pi)^{2}}\right] \\
& B(-2,1)=188 / 3-\frac{1}{(2 \pi)^{2}} \frac{736}{9}-\frac{632}{3} Z_{1}-\frac{224}{3} Z_{0} \\
& B(-2,2)=-11+\frac{24}{(2 \pi)^{2}}+114 Z_{1}+24 Z_{0} \\
& B(-2,3)=\frac{3}{4}-3 Z_{1}+6 Z_{0} .
\end{aligned}
$$

At $p \leq 3, \mathcal{B}\left(p, q ; \mu_{B}^{2}\right)$ functions involve no divergencies: $\mathcal{B}\left(p, q ; \mu_{B}^{2}\right)=B(p, q)=\mathcal{B}(p, q ; 0)$. They can be determined by the recurrent relations presented in Appendix 3. The initial conditions are provided by the formulas (69) and $B$ functions calculated previously.

To express $B(r, 1)$ or $B(r, 2)$ or $B(r, 3)$ at $r \leq 3$ in terms of $F_{0}, Z_{0}$, and $Z_{1}$, one should apply these relations beginning with $p=r$ and ending with $p=-3$. In so doing, one must know $B(p, q)$ at $q=0,-1,-2$ and $r \leq p \leq 0$

- $\mathcal{B}$ functions at $p \leq-1, q \geq 4$

$\mathcal{B}$ functions in this domain are computed by the recurrent relations presented in the Appendix 4. The initial conditions for these relations are provided by $\mathcal{B}\left(0, q ; \mu_{B}^{2}\right)$ as well as $\mathcal{B}\left(p, q ; \mu_{B}^{2}\right)$ at $p \leq-1, q \leq 3$ presented above.

\subsection{2 $\mathcal{J}$ functions.}

Here, the quantities $J(p, q)$ introduced in (58) are expressed in terms of the boson constants $Z_{1}, Z_{0}, F_{0}$ introduced in the previous Section, and the quantities $Y_{0} \div Y_{11}$ defined by the relations

$$
\begin{aligned}
Y_{4}=\frac{J(1,0)}{2}, & Y_{5}=J(1,-1), & Y_{6}=2 J(1,-2), \\
Y_{7}=\frac{J(2,-1)}{2}, & Y_{8}=J(2,-2), & Y_{9}=\frac{J(3,-2)}{2}, \\
Y_{10}=J(3,-3), & Y_{11}=2 J(3,-4), & Y_{0}=\frac{J(2,0)}{4}-\frac{F_{0}}{16 \pi^{2}},
\end{aligned}
$$

and

$$
\begin{aligned}
Y_{1}= & \frac{1}{48}-\frac{1}{4} Z_{0}-\frac{1}{24} J(-1,2)+\frac{1}{12} J(0,1)+\frac{1}{12} J(1,0) ; \\
Y_{2}= & \frac{1}{6}-\frac{1}{\pi^{2}}-Z_{0}-\frac{1}{6} J(-1,2)+\frac{1}{3} J(0,1)-\frac{1}{24} J(1,-2)-\frac{1}{12} J(1,-1)- \\
& -\frac{17}{8} J(1,0)+4 J(1,1)-\frac{1}{48} J(2,-2)+\frac{25}{6} J(2,-1)-4 J(2,0) ; \\
Y_{3}= & -\frac{1}{384 \pi^{2}}-F_{0} \frac{1}{128 \pi^{2}}+\frac{1}{96} Z_{0}-\frac{1}{48} J(-1,3)+\frac{1}{192} J(0,1)+\frac{1}{48} J(0,2)+\frac{1}{48} J(1,1) ;
\end{aligned}
$$




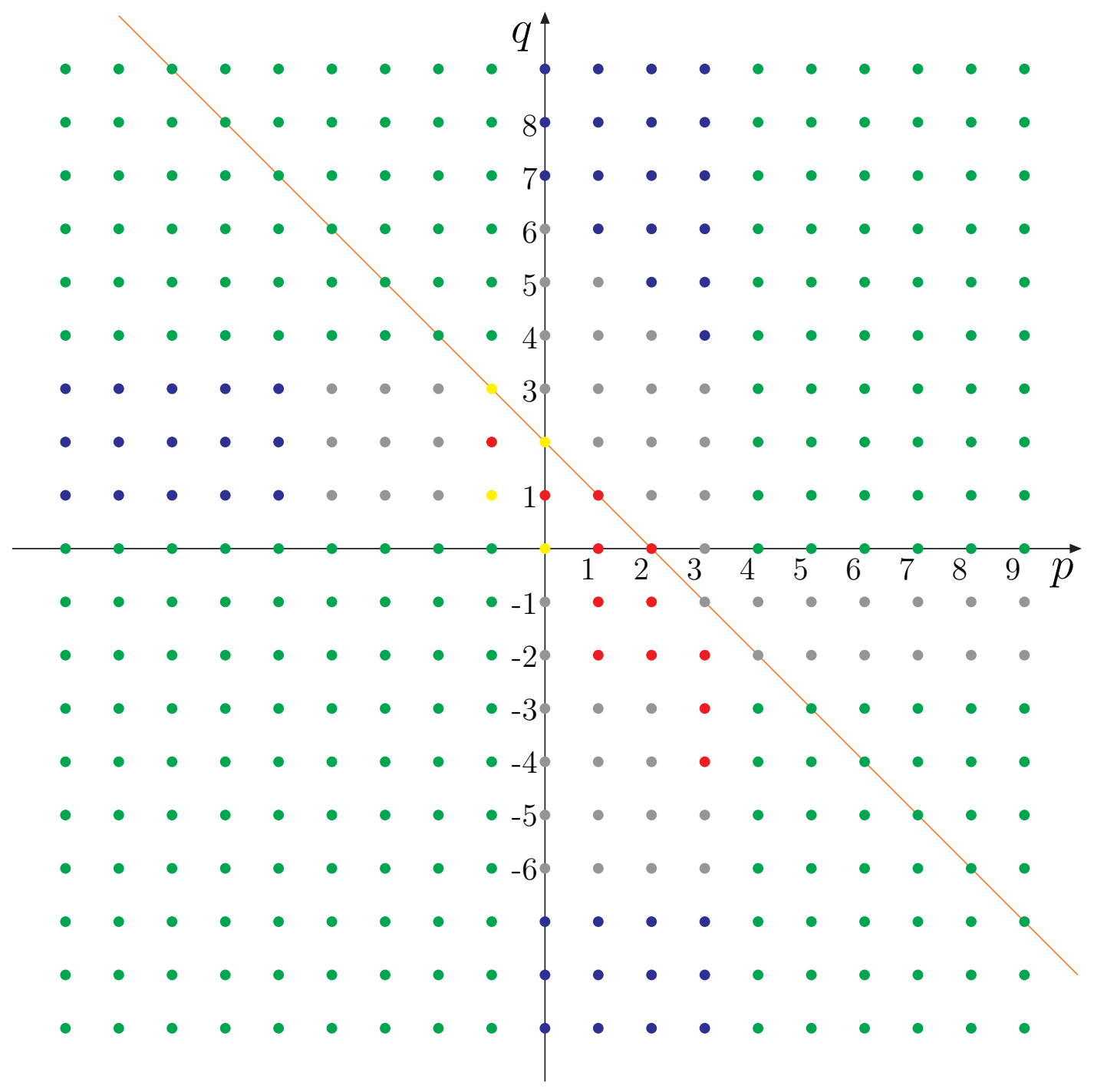

Figure 1: Values of $(p, q)$ at which the functions $G\left(p, q ; \mu_{B}^{2}\right)$ are calculated are shown by dots. The 12 fermionic basic constants are determined from the functions associated with red dots; yellow dots show the constants $X_{0} \div X_{3}$ that appear in the expressions for $G\left(p, q ; \mu_{B}^{2}\right)$ but cancel in the expressions for Feynman integrals. Functions shown by gray are calculated explicitly and given in the formulas (69), (170), (71), (73), (174), and (75), which provide the initial conditions for the recurrent relations. The order of implementation of the recurrent relations is as follows: 1 . $\mathcal{B}$ functions are calculated at $p=0$, then at $p<0, q \leq 0$, then over the left strip, then at $p<0, q \geq 4$. 2. $\mathcal{J}$ functions are calculated in the "down" strip, then lower-left domain (shown by green), then "left" strip, then "up" strip then up-left domain, then up-right domain, then down-right domain. 
and the quantities

$$
\begin{array}{cc}
X_{0}=J(-1,1), & X_{1}=J(-1,3), \\
X_{2}=J(0,0), & X_{3}=J(0,2) .
\end{array}
$$

as well. The numerical values of the quantities $X_{0}, X_{1}, X_{2}$, and $X_{3}$ are not needed because expressions for the integrals (51) in terms of $G(p, q)$ etc. do not involve them. As for the other constants, they are well known (the table below is taken from the review [1], notation $\mathcal{F}(p, q)$ is borrowed from there):

\begin{tabular}{|c|r|}
\hline$Y_{0}$ & -0.01849765846791657356 \\
$Y_{1}$ & 0.00376636333661866811 \\
$Y_{2}$ & 0.00265395729487879354 \\
$Y_{3}$ & 0.00022751540615147107 \\
$Y_{4}=\mathcal{F}(1,0)$ & 0.08539036359532067914 \\
$Y_{5}=\mathcal{F}(1,-1)$ & 0.46936331002699614475 \\
$Y_{6}=\mathcal{F}(1,-2)$ & 3.39456907367713000586 \\
$Y_{7}=\mathcal{F}(2,-1)$ & 0.05188019503901136636 \\
$Y_{8}=\mathcal{F}(2,-2)$ & 0.23874773756341478520 \\
$Y_{9}=\mathcal{F}(3,-2)$ & 0.03447644143803223145 \\
$Y_{10}=\mathcal{F}(3,-3)$ & 0.13202727122781293085 \\
$Y_{11}=\mathcal{F}(3,-4)$ & 0.75167199030295682254 \\
\hline
\end{tabular}

Table 1: New constants appearing in the general fermionic case.

- Thus $\mathcal{J}$ functions over the domain $\mathcal{A}=\{(p, q): 0 \leq p \leq 3,-6 \leq p \leq 6-p\} \cup$

$\cup\{(p, q):-4 \leq p \leq-1,1 \leq q \leq 3\}$ calculated by the procedure indicated in [5] can be represented in terms of the above constants as follows:

$$
\begin{aligned}
& J(0,0)=X_{2} ; \\
& J(0,-1)=4 X_{2}+315 Y_{10}-1218 Y_{9}-134 Y_{8}+804 Y_{7}-2 Y_{6}+\frac{25}{2} Y_{5}-114 Y_{4}+\frac{15}{(2 \pi)^{2}} ; \\
& J(0,-2)=-443 / 12+18 X_{2}+525 / 16 Y_{11}+5265 / 4 Y_{10}-7661 / 2 Y_{9}-8173 / 12 Y_{8} \\
& \quad+2085 Y_{7}-7 / 48 Y_{6}+1657 / 24 Y_{5}-339 / 2 Y_{4}+35 / 4(2 \pi)^{-2} ; \\
& J(0,-3)=-1549 / 27+88 X_{2}+595 / 4 Y_{11}+92395 / 6 Y_{10}-487883 / 9 Y_{9}-197384 / 27 Y_{8} \\
& \quad+103330 / 3 Y_{7}-6569 / 108 Y_{6}+57949 / 108 Y_{5}-12829 / 3 Y_{4}+9029 / 18(2 \pi)^{-2} ; \\
& J(0,-4)=-1024087 / 576+917 / 2 X_{2}+1919221 / 768 Y_{11}+7343317 / 192 Y_{10} \\
& \quad-20889553 / 288 Y_{9}-14398667 / 576 Y_{8}+3075865 / 144 Y_{7}+325123 / 2304 Y_{6} \\
& \quad+2538989 / 1152 Y_{5}+501607 / 96 Y_{4}-910433 / 576(2 \pi)^{-2} ; \\
& J(0,-5)=22816157 / 32400+2514 X_{2}+14234765 / 4032 Y_{11}+6270202009 / 8400 Y_{10} \\
& \quad-171277520509 / 63000 Y_{9}-11273402747 / 32400 Y_{8}+33415313987 / 18900 Y_{7} \\
& \quad-402215297 / 129600 Y_{6}+52200958189 / 2268000 Y_{5}-4825909001 / 21000 Y_{4}
\end{aligned}
$$




$$
\begin{aligned}
& +3567631667 / 126000(2 \pi)^{-2} \\
& J(0,-6)=-59060175671 / 583200+14376 X_{2}-456897556151 / 2268000(2 p i)^{-2} \\
& +290748296317 / 1814400 Y_{11}-46846789343 / 252000 Y_{10}+1099242273317 / 226800 Y_{9} \\
& -1709413667089 / 4082400 Y_{8}-8051167629571 / 1701000 Y_{7}+41141268191 / 2332800 Y_{6} \\
& +1776831395699 / 40824000 Y_{5}+61514640887 / 54000 Y_{4} \text {; } \\
& J(1,0)=2 Y_{4} \text {; } \\
& J(1,-1)=Y_{5} \text {; } \\
& J(1,-2)=1 / 2 Y_{6} \text {; } \\
& J(1,-3)=52 / 3+683 / 2 Y_{10}-1342 Y_{9}-439 / 3 Y_{8}+924 Y_{7} \\
& -13 / 3 Y_{6}+89 / 12 Y_{5}-132 Y_{4}+17(2 \pi)^{-2} \text {; } \\
& J(1,-4)=-761 / 9+683 / 12 Y_{11}-10807 / 3 Y_{10}+48538 / 3 Y_{9}+12539 / 9 Y_{8} \\
& -35284 / 3 Y_{7}+1481 / 36 Y_{6}-887 / 18 Y_{5}+1874 Y_{4}-803 / 3(2 \pi)^{-2} \text {; } \\
& J(1,-5)=34745 / 27-52147 / 63 Y_{11}+6069519 / 140 Y_{10}-27087257 / 140 Y_{9} \\
& -450260 / 27 Y_{8}+88135577 / 630 Y_{7}-42845 / 108 Y_{6}+2730857 / 3780 Y_{5} \\
& -3125867 / 140 Y_{4}+541505 / 168(2 \pi)^{-2} \text {; } \\
& J(1,-6)=-3470071 / 324+123774917 / 10800 Y_{11}-15164760473 / 31500 Y_{10} \\
& +34075577647 / 15750 Y_{9}+10210300231 / 56700 Y_{8}-36997574549 / 23625 Y_{7} \\
& +5205091 / 1296 Y_{6}-4977314411 / 567000 Y_{5}+1327628299 / 5250 Y_{4} \\
& -232435501 / 6300(2 \pi)^{-2} \\
& J(2,0)=4 Y_{0}+F_{0}(2 \pi)^{-2} \text {; } \\
& J(2,-1)=2 Y_{7} \text {; } \\
& J(2,-2)=Y_{8} \text {; } \\
& J(2,-3)=-Y_{10}+90 Y_{9}-2 Y_{8}-84 Y_{7}+5 / 2 Y_{5}+18 Y_{4}-3(2 \pi)^{-2} \text {; } \\
& J(2,-4)=-13 / 3-1 / 4 Y_{11}+Y_{10}-326 Y_{9}+13 / 3 Y_{8}+300 Y_{7}+31 / 12 Y_{6} \\
& -19 / 6 Y_{5}-66 Y_{4}+13(2 \pi)^{-2} \text {; } \\
& J(2,-5)=1012 / 9+53 / 63 Y_{11}+519787 / 210 Y_{10}-316251 / 35 Y_{9}-3217 / 3 Y_{8} \\
& +214918 / 35 Y_{7}-343 / 9 Y_{6}+17621 / 420 Y_{5}-29051 / 35 Y_{4}+3859 / 42(2 \pi)^{-2} \text {; } \\
& J(2,-6)=-48007 / 36+8466287 / 15120 Y_{11}-272218199 / 6300 Y_{10}+593087111 / 3150 Y_{9} \\
& +64920421 / 3780 Y_{8}-30568297 / 225 Y_{7}+70867 / 144 Y_{6}-22014731 / 37800 Y_{5} \\
& +7424029 / 350 Y_{4}-3722933 / 1260(2 \pi)^{-2} \text {; } \\
& J(3,0)=-35 / 256 Y_{11}-57 / 64 Y_{10}-301 / 2304 Y_{9}+85 / 96 Y_{8}+1405 / 1152 Y_{7} \\
& -1 / 384 Y_{5}-461 / 768 Y_{4}-2 Y_{0}+\left(1433 / 1536-1 / 2 F_{0}\right)(2 \pi)^{-2} \text {; } \\
& J(3,-1)=155 / 96 Y_{9}-83 / 48 Y_{7}+11 / 32 Y_{4}+4 Y_{0}+\left(F_{0}-31 / 64\right)(2 \pi)^{-2} \text {; } \\
& J(3,-2)=2 Y_{9} \text {; } \\
& J(3,-3)=Y_{10} \text {; } \\
& J(3,-4)=1 / 2 Y_{11} \text {; } \\
& J(3,-5)=-85 / 42 Y_{11}-1649 / 140 Y_{10}+15548 / 35 Y_{9}+7 / 6 Y_{8}-14264 / 35 Y_{7} \\
& +5609 / 840 Y_{5}+2943 / 35 Y_{4}-106 / 7(2 \pi)^{-2} \text {; }
\end{aligned}
$$




$$
\begin{aligned}
& J(3,-6)=-224 / 9+2812 / 945 Y_{11}+47669 / 1575 Y_{10}-595726 / 225 Y_{9}+6854 / 945 Y_{8} \\
& +3804196 / 1575 Y_{7}+101 / 9 Y_{6}-173539 / 9450 Y_{5}-90298 / 175 Y_{4}+33203 / 315(2 \pi)^{-2} \text {; } \\
& J(-1,1)=X_{0} \\
& J(-2,1)=-1135 / 9-158 / 9 X_{0}+448 / 3 X_{3}+722 / 9 X_{2}-448 / 3 X_{1}+1295 / 12 Y_{11} \\
& +56237 / 9 Y_{10}-61066 / 3 Y_{9}-83036 / 27 Y_{8}+108068 / 9 Y_{7}-283 / 18 Y_{6} \\
& +15217 / 54 Y_{5}-1274 Y_{4}-19456 / 3 Y_{3}-32 / 3 Y_{2}+1472 / 3 Y_{1}+896 / 3 Y_{0} \\
& +865 / 9(2 \pi)^{-2}+64 / 9 F_{0}(2 \pi)^{-2}+536 / 3 Z_{1}+5488 / 27 Z_{0} \text {; } \\
& J(-3,1)=11845003 / 15000+105068 / 375 X_{0}-371584 / 125 X_{3}-200897 / 375 X_{2} \\
& +371584 / 125 X_{1}+204127 / 160 Y_{11}+19090453 / 600 Y_{10}-133042333 / 1500 Y_{9} \\
& -175667117 / 9000 Y_{8}+39223253 / 750 Y_{7}-214589 / 12000 Y_{6}+8996881 / 18000 Y_{5} \\
& -207851 / 60 Y_{4}+15388672 / 125 Y_{3}+27568 / 125 Y_{2}-1398848 / 125 Y_{1} \\
& -640512 / 125 Y_{0}+6221759 / 15000(2 \pi)^{-2}-153472 / 375 F_{0}(2 \pi)^{-2} \\
& -2696248 / 625 Z_{1}-24764192 / 5625 Z_{0} \text {; } \\
& J(-4,1)=-3329832752387 / 64827000-117549296 / 25725 X_{0}+446453248 / 8575 X_{3} \\
& +566923184 / 25725 X_{2}-446453248 / 8575 X_{1}+737380289 / 14112 Y_{11} \\
& +242892361651 / 123480 Y_{10}-5552574235627 / 926100 Y_{9} \\
& -640505149891 / 617400 Y_{8}+1563368068291 / 463050 Y_{7}-23776321823 / 7408800 Y_{6} \\
& +321309701383 / 3704400 Y_{5}-89421147379 / 308700 Y_{4}-17930338304 / 8575 Y_{3} \\
& -92940608 / 25725 Y_{2}+1782287616 / 8575 Y_{1}+2138417152 / 25725 Y_{0} \\
& +991072905679 / 64827000(2 \pi)^{-2}+4988416 / 525 F_{0}(2 \pi)^{-2} \\
& +25248697568 / 300125 Z_{1}+73082913664 / 900375 Z_{0} \\
& J(-1,2)=2 / 3-8 X_{3}+8 X_{1}-1 / 24 Y_{8}+50 / 3 Y_{7}-1 / 24 Y_{6}-1 / 6 Y_{5}-35 / 6 Y_{4} \\
& +384 Y_{3}-2 Y_{2}-16 Y_{1}-32 Y_{0}+4\left(F_{0}-1\right)(2 \pi)^{-2}-10 Z_{0} \text {; } \\
& J(-2,2)=135 / 4+19 / 2 X_{0}-48 X_{3}-41 / 2 X_{2}+48 X_{1}-105 / 16 Y_{11}-1851 / 4 Y_{10} \\
& +3075 / 2 Y_{9}+827 / 4 Y_{8}-841 Y_{7}+11 / 8 Y_{6}-269 / 8 Y_{5}+175 / 2 Y_{4}+2304 Y_{3} \\
& +12 Y_{2}-96 Y_{1}-96 Y_{0}-45 / 4(2 \pi)^{-2}-114 Z_{1}-76 Z_{0} \text {; } \\
& J(-3,2)=-29723 / 16-2365 / 9 X_{0}+7712 / 3 X_{3}+32881 / 36 X_{2}-7712 / 3 X_{1} \\
& +56805 / 64 Y_{11}+6355039 / 144 Y_{10}-5035139 / 36 Y_{9}-4682939 / 216 Y_{8} \\
& +1411847 / 18 Y_{7}-4543 / 48 Y_{6}+2051831 / 864 Y_{5}-68044 / 9 Y_{4}-330752 / 3 Y_{3} \\
& -252 Y_{2}+26992 / 3 Y_{1}+13952 / 3 Y_{0}+\left(54773 / 72+2336 / 9 F_{0}\right)(2 \pi)^{-2} \\
& +13706 / 3 Z_{1}+111704 / 27 Z_{0} \\
& J(-4,2)=492009959 / 12000+87748 / 15 X_{0}-324224 / 5 X_{3}-275752 / 15 X_{2}+324224 / 5 X_{1} \\
& -3344999 / 384 Y_{11}-240354739 / 480 Y_{10}+5720706571 / 3600 Y_{9} \\
& +329440181 / 1440 Y_{8}-302115271 / 360 Y_{7}+2092337 / 1920 Y_{6}-518442119 / 14400 Y_{5} \\
& +285938617 / 3600 Y_{4}+2650112 Y_{3}+75952 / 15 Y_{2}-1259712 / 5 Y_{1}-318976 / 3 Y_{0} \\
& -\left(423392089 / 36000+159872 / 15 F_{0}\right)(2 \pi)^{-2}-44526248 / 375 Z_{1}-40322528 / 375 Z_{0} \text {; } \\
& J(-1,3)=X_{1} \text {; } \\
& J(-2,3)=1 / 12-1 / 4 X_{0}-12 X_{3}+X_{2}+12 X_{1}+105 / 2 Y_{10}-203 Y_{9}-1075 / 48 Y_{8}
\end{aligned}
$$


$+159 Y_{7}-19 / 48 Y_{6}+23 / 12 Y_{5}-103 / 4 Y_{4}+576 Y_{3}-7 Y_{2}-60 Y_{1}$

$-48 Y_{0}-7 / 2(2 \pi)^{-2}+6 F_{0}(2 \pi)^{-2}+12 Z_{1}-19 Z_{0}$

$J(-3,3)=871 / 2+629 / 9 X_{0}-1696 / 3 X_{3}-1778 / 9 X_{2}+1696 / 3 X_{1}-175 / 2 Y_{11}$

$-50840 / 9 Y_{10}+167119 / 9 Y_{9}+279089 / 108 Y_{8}-91946 / 9 Y_{7}+187 / 12 Y_{6}$

$-10154 / 27 Y_{5}+9446 / 9 Y_{4}+77824 / 3 Y_{3}+116 Y_{2}-4784 / 3 Y_{1}-3136 / 3 Y_{0}$

$-1739 / 6(2 \pi)^{-2}-304 / 9 F_{0}(2 \pi)^{-2}-1502 Z_{1}-28456 / 27 Z_{0}$;

$J(-4,3)=-40263778 / 1875-2997542 / 1125 X_{0}+10588096 / 375 X_{3}+20165461 / 2250 X_{2}$

$-10588096 / 375 X_{1}+602637 / 80 Y_{11}+161298737 / 450 Y_{10}$

$-5056485013 / 4500 Y_{9}-4717685677 / 27000 Y_{8}+1381579093 / 2250 Y_{7}$

$-8668453 / 12000 Y_{6}+284825729 / 13500 Y_{5}-50984761 / 900 Y_{4}$

$-444755968 / 375 Y_{3}-347064 / 125 Y_{2}+39247712 / 375 Y_{1}+17916928 / 375 Y_{0}$

$+182925853 / 15000(2 \pi)^{-2}+4427968 / 1125 F_{0}(2 \pi)^{-2}+38504584 / 625 Z_{1}$

$+860640608 / 16875 Z_{0}$;

$J(0,1)=1 / 12-4 X_{3}+4 X_{1}-1 / 48 Y_{8}+25 / 3 Y_{7}-1 / 48 Y_{6}-1 / 12 Y_{5}-59 / 12 Y_{4}$

$+192 Y_{3}-Y_{2}+4 Y_{1}-16 Y_{0}+\left(2 F_{0}-2\right)(2 \pi)^{-2}-2 Z_{0}$;

$J(0,2)=X_{3}$

$J(0,3)=91 / 1024+1 / 384 X_{0}+1 / 4 X_{3}-1 / 96 X_{2}-35 / 768 Y_{11}-27 / 32 Y_{10}+109 / 72 Y_{9}$

$+19229 / 36864 Y_{8}+383 / 2304 Y_{7}-11 / 4096 Y_{6}-443 / 9216 Y_{5}-1243 / 3072 Y_{4}$

$-3 / 2 Y_{3}-33 / 256 Y_{2}+17 / 64 Y_{1}-33 / 16 Y_{0}-13 / 72(2 \pi)^{-2}$

$-5 / 48 F_{0}(2 \pi)^{-2}-1 / 4 Z_{1}-49 / 192 Z_{0}$;

$J(0,4)=80441 / 2211840+31 / 27648 X_{0}+17 / 288 X_{3}-31 / 6912 X_{2}+1 / 288 X_{1}$

$+161 / 24576 Y_{11}-17767 / 92160 Y_{10}+23 / 32 Y_{9}+161077 / 2949120 Y_{8}$

$-10667 / 552960 Y_{7}-14369 / 8847360 Y_{6}-16271 / 737280 Y_{5}-343291 / 2211840 Y_{4}$

$-1379 / 1440 Y_{3}-14369 / 184320 Y_{2}+9409 / 46080 Y_{1}-8609 / 11520 Y_{0}$

$+\left(539 / 17280 F_{0}-18031 / 86400\right)(2 \pi)^{-2}-237 / 2560 Z_{1}-965 / 9216 Z_{0}$;

$J(0,5)=7632781 / 594542592+523 / 1327104 X_{0}+209 / 13824 X_{3}-523 / 331776 X_{2}$

$+25 / 13824 X_{1}+77651 / 42467328 Y_{11}-1253633 / 17694720 Y_{10}+56974703 /$

$159252480 Y_{9}+782803057 / 35672555520 Y_{8}+34653559 / 2229534720 Y_{7}-$

$10633541 / 11890851840 Y_{6}-80793859 / 8918138880 Y_{5}-291120383 / 2972712960 Y_{4}$

$-262597 / 645120 Y_{3}-10633541 / 247726080 Y_{2}+8290501 / 61931520 Y_{1}-5150021 / 15482880 Y_{0}$

$+\left(43529 / 5806080 F_{0}-8543128133 / 78033715200\right)(2 \pi)^{-2}$

$-569479 / 20643840 Z_{1}-2292133 / 61931520 Z_{0}$;

$J(0,6)=1133309347 / 237817036800+1429 / 10616832 X_{0}+2371 / 552960 X_{3}-1429 / 2654208 X_{2}$

$+401 / 552960 X_{1}+10571051 / 10192158720 Y_{11}-639402031 / 29727129600 Y_{10}$

$+48006407249 / 267544166400 Y_{9}+5666277343 / 1223059046400 Y_{8}+2239163267 / 107017666560 Y_{7}$

$-470661551 / 951268147200 Y_{6}-1645375871 / 428070666240 Y_{5}-40565004503 / 713451110400 Y_{4}$

$-23258381 / 154828800 Y_{3}-470661551 / 19818086400 Y_{2}+406642351 / 4954521600 Y_{1}$

$-153477551 / 1238630400 Y_{0}+\left(1169977 / 232243200 F_{0}-1234692078509 / 18728091648000\right)(2 \pi)^{-2}$ 
-117647549/14863564800 $Z_{1}-202376477 / 14863564800 Z_{0}$;

$$
\begin{aligned}
& J(1,1)=-1 / 48+1 / 192 Y_{8}-25 / 12 Y_{7}+1 / 192 Y_{6}+1 / 48 Y_{5}+59 / 48 Y_{4} \\
& \quad+1 / 4 Y_{2}-Y_{1}+4 Y_{0}+\left(1+F_{0}\right)(2 \pi)^{-2} ; \\
& J(1,2)=-19 / 1536-35 / 768 Y_{11}-19 / 64 Y_{10}-173 / 288 Y_{9}+5497 / 18432 Y_{8} \\
& \quad-293 / 1152 Y_{7}+19 / 6144 Y_{6}+53 / 4608 Y_{5}+187 / 512 Y_{4}+Y_{3}+19 / 128 Y_{2} \\
& \quad-19 / 32 Y_{1}+3 / 8 Y_{0}+307 / 576(2 \pi)^{-2}+1 / 96 Z_{0} ; \\
& J(1,3)=-5491 / 737280+721 / 73728 Y_{11}+1957 / 30720 Y_{10}-21361 / 55296 Y_{9} \\
& \quad-542443 / 8847360 Y_{8}-266629 / 552960 Y_{7}+5959 / 2949120 Y_{6}+18289 / 2211840 Y_{5} \\
& \quad+274901 / 737280 Y_{4}+149 / 480 Y_{3}+5959 / 61440 Y_{2}-5959 / 15360 Y_{1} \\
& \quad+1799 / 3840 Y_{0}+\left(438551 / 2764800+11 / 160 F_{0}\right)(2 \pi)^{-2}-13 / 5120 Z_{1}+35 / 3072 Z_{0} ; \\
& J(1,4)=-135181 / 27525120-1519 / 3538944 Y_{11}-4123 / 1474560 Y_{10}-2532143 / 13271040 Y_{9} \\
& \quad+12187051 / 2972712960 Y_{8}-12605351 / 37158912 Y_{7}+1307897 / 990904320 Y_{6} \\
& \quad+783523 / 148635648 Y_{5}+18292649 / 82575360 Y_{4}-533 / 161280 Y_{3} \\
& \quad+1307897 / 20643840 Y_{2}-1307897 / 5160960 Y_{1}+93817 / 1290240 Y_{0} \\
& \quad+\left(346641047 / 2167603200-1 / 1680 F_{0}\right)(2 \pi)^{-2}-22817 / 15482880 Z_{1}+146059 / 15482880 Z_{0} ; \\
& J(1,5)=-32271257 / 9512681472+9401131 / 2038431744 Y_{11}+178621489 / 5945425920 Y_{10} \\
& \quad-1051530899 / 53508833280 Y_{9}-49631503063 / 1712282664960 Y_{8} \\
& \quad-5652301063 / 15288238080 Y_{7}+167849953 / 190253629440 Y_{6} \\
& \quad+1548254101 / 428070666240 Y_{5}+26366380697 / 142690222080 Y_{4} \\
& \quad-4481117 / 30965760 Y_{3}+167849953 / 3963617280 Y_{2}-167849953 / 990904320 Y_{1} \\
& \quad+9544673 / 247726080 Y_{0}+\left(260638894247 / 3745618329600+1339 / 215040 F_{0}\right)(2 \pi)^{-2} \\
& \quad-541139 / 990904320 Z_{1}+806521 / 110100480 Z_{0} ; \\
& \quad+(21)
\end{aligned}
$$$$
J(2,1)=23 / 4608-35 / 384 Y_{11}-19 / 32 Y_{10}-173 / 144 Y_{9}+3619 / 6144 Y_{8}
$$$$
+2827 / 1152 Y_{7}-23 / 18432 Y_{6}-31 / 4608 Y_{5}-3949 / 4608 Y_{4}+Y_{3}-23 / 384 Y_{2}
$$$$
+23 / 96 Y_{1}-19 / 24 Y_{0}+\left(127 / 288-1 / 4 F_{0}\right)(2 \pi)^{-2}-1 / 96 Z_{0} \text {; }
$$$$
J(2,2)=7043 / 1105920+343 / 12288 Y_{11}+931 / 5120 Y_{10}-3731 / 4608 Y_{9}
$$$$
-806147 / 4423680 Y_{8}+339959 / 276480 Y_{7}-6467 / 4423680 Y_{6}-5879 / 1105920 Y_{5}
$$$$
-365953 / 1105920 Y_{4}+221 / 240 Y_{3}-6467 / 92160 Y_{2}+6467 / 23040 Y_{1}
$$$$
+4373 / 5760 Y_{0}+\left(23 / 160 F_{0}-25657 / 76800\right)(2 \pi)^{-2}-1 / 480 Z_{1}-7 / 512 Z_{0} \text {; }
$$$$
J(2,3)=4611371 / 743178240-73045 / 3538944 Y_{11}-39653 / 294912 Y_{10}
$$$$
-12722017 / 13271040 Y_{9}+393115913 / 2972712960 Y_{8}+103603669 / 61931520 Y_{7}
$$$$
-4248887 / 2972712960 Y_{6}-504563 / 82575360 Y_{5}-79536305 / 148635648 Y_{4}
$$$$
+155081 / 161280 Y_{3}-4248887 / 61931520 Y_{2}+4248887 / 15482880 Y_{1}
$$$$
+66473 / 3870720 Y_{0}+493568683 / 6502809600(2 \pi)^{-2}
$$$$
-1093 / 26880 F_{0}(2 \pi)^{-2}-10069 / 5160960 Z_{1}-74813 / 5160960 Z_{0} \text {; }
$$$$
J(2,4)=196602193 / 35672555520+3693635 / 509607936 Y_{11}+14035813 / 297271296 Y_{10}
$$$$
-12442566511 / 13377208320 Y_{9}-20639077343 / 428070666240 Y_{8}
$$$$
+36967799143 / 26754416640 Y_{7}-181900981 / 142690222080 Y_{6}
$$ 


$$
\begin{aligned}
& -530928403 / 107017666560 Y_{5}-2755058051 / 7134511104 Y_{4}+2424601 / 2580480 Y_{3} \\
& -181900981 / 2972712960 Y_{2}+181900981 / 743178240 Y_{1}+46110859 / 185794560 Y_{0} \\
& \quad-79216495853 / 936404582400(2 \pi)^{-2}+4141 / 215040 F_{0}(2 \pi)^{-2} \\
& \quad-408367 / 247726080 Z_{1}-125077 / 9175040 Z_{0} ; \\
& J(3,1)=-2473 / 2211840+1687 / 24576 Y_{11}+4579 / 10240 Y_{10}+28153 / 27648 Y_{9} \\
& \quad-3930323 / 8847360 Y_{8}-866969 / 552960 Y_{7}+2797 / 8847360 Y_{6}+5689 / 2211840 Y_{5} \\
& \quad+1137263 / 2211840 Y_{4}-211 / 480 Y_{3}+2797 / 184320 Y_{2}-2797 / 46080 Y_{1} \\
& \quad+12317 / 11520 Y_{0}+\left(23 / 80 F_{0}-323129 / 460800\right)(2 \pi)^{-2}-3 / 5120 Z_{1}+11 / 3072 Z_{0} ; \\
& J(3,2)=-326465 / 148635648-206605 / 3538944 Y_{11}-112157 / 294912 Y_{10} \\
& \quad+2493365 / 2654208 Y_{9}+225122965 / 594542592 Y_{8}-27027877 / 37158912 Y_{7} \\
& \quad+336725 / 594542592 Y_{6}+19049 / 16515072 Y_{5}+6739903 / 148635648 Y_{4} \\
& \quad-22955 / 32256 Y_{3}+336725 / 12386304 Y_{2}-336725 / 3096576 Y_{1}-483563 / 774144 Y_{0} \\
& \quad+\left(429664553 / 1300561920-331 / 2688 F_{0}\right)(2 \pi)^{-2}-95 / 344064 Z_{1}+6823 / 1032192 Z_{0} ; \\
& J(3,3)=-69355327 / 23781703680+4168243 / 339738624 Y_{11}+79196617 / 990904320 Y_{10} \\
& \quad+3687114839 / 2972712960 Y_{9}-22463493247 / 285380444160 Y_{8} \\
& \quad-1869533095 / 1189085184 Y_{7}+70582891 / 95126814720 Y_{6} \\
& \quad+45684329 / 14269022208 Y_{5}+362000483 / 880803840 Y_{4}-1586311 / 1720320 Y_{3} \\
& \quad+70582891 / 1981808640 Y_{2}-70582891 / 495452160 Y_{1}+7231211 / 123863040 Y_{0} \\
& \quad-131572132177 / 624269721600(2 \pi)^{-2}+12241 / 215040 F_{0}(2 \pi)^{-2} \\
& \quad-34099 / 165150720 Z_{1}+1460153 / 165150720 Z_{0} ;
\end{aligned}
$$

- $\mathcal{J}\left(p, q ; \mu_{B}^{2}\right)$ functions at $0 \leq p \leq 3, q \leq-7$

Provided that $\mathcal{J}\left(p, q ; \mu_{B}^{2}\right)$ over the domain $\mathcal{A}$ (at $\left.q \leq 0\right)$ and $B(0, q)$ at $q \leq 0$ are known, $\mathcal{J}$ functions over this strip can be found by the recurrent relations in Appendix 5. Note that there are no divergent terms here.

- $\mathcal{J}\left(p, q ; \mu_{B}^{2}\right)$ functions at $p \leq-1, q \leq 0$

Given $\mathcal{J}$ functions over the strip $0 \leq p \leq 3, q \leq 0$ and $\mathcal{B}$ functions at $p \leq 0, q \leq 0, \mathcal{J}$ functions at $p \leq-1, q \leq 0$ can be determined using the recurrent relations in Appendix 8, however, with $\mu_{B}=0$ (in this domain, $\mathcal{J}\left(p, q ; \mu_{B}^{2}\right)=J(p, q)$ ).

- $\mathcal{J}\left(p, q ; \mu_{B}^{2}\right)$ functions at $p \leq-5,1 \leq q \leq 3$.

They can be determined using the recurrent relations presented in Appendix 6. The initial conditions are provided by the formulas (74) and $B$ functions determined previously. In calculation of $J(p, q)$ one needs $B(r, s)$ at $r \geq p$ and $-3 \leq q \leq 3$.

- $\mathcal{J}\left(p, q ; \mu_{B}^{2}\right)$ functions at $0 \leq p \leq 3$ and $q \geq 6-p$.

In this domain, the divergent part $D(p, q)$ is calculated separately, the results are partially presented in (85); thus we calculate the functions $J(p, q)$ by the formulas 


$$
J(n, q+1-n)=\frac{1}{(2 q-1)(2 q-3)} \sum_{k=0}^{3} M(n, k, q) Z_{k}(q),
$$

where $M(n, k, q)$ are given by

$$
\begin{aligned}
& M(0,0, q)=(q+1) / 32 /(q-1) ; \\
& M(0,1, q)=-(8 q-3)(q-2) / 32 / q /(q-1) ; \\
& M(0,2, q)=-(q-2) / 4 / q /(q-1) ; \\
& M(0,3, q)=-(q-2) / 2 / q /(q-1)^{2} ; \\
& M(1,0, q)=(q+1) / 32 ; \\
& M(1,1, q)=3 / 32 ; \\
& M(1,2, q)=-(q-2) / 4 / q ; \\
& M(1,3, q)=-(q-2) / 2 / q /(q-1) ; \\
& M(2,0, q)=q(q+1) / 32 ; \\
& M(2,1, q)=3 q / 32 ; \\
& M(2,2, q)=3 / 16 / q ; \\
& M(2,3, q)=-(q-2) / 2 /(q-1) ; \\
& M(3,0, q)=q(q+1)^{2} / 64 ; \\
& M(3,1, q)=3 q(q+1) / 64 ; \\
& M(3,2, q)=3(q+1) / 32 / q ; \\
& M(3,3, q)=-\left(8 q^{2}-19 q+3\right) / 16 / q /(q-1)
\end{aligned}
$$

and $Z_{k}(q)$ can be determined from the recurrent relations presented in Appendix 7. The initial conditions are provided by the formulas (73), (74), and (75); $\mathcal{B}(0, q)$ at $q>0$ and $D(p, q, r)$ at $0 \leq p \leq 3, q>1-p, 0 \leq r \leq p+q-2$ are also needed.

- $\mathcal{J}\left(p, q ; \mu_{B}^{2}\right)$ functions at $p \leq-1, q \geq 4$.

The respective recurrent relations can be found in Appendix 8, the initial conditions are provided by the $\mathcal{B}(p, q)$ functions at $p \leq 0$ and $q \geq-2$ and $\mathcal{J}(p, q)$ functions at $0 \leq p \leq 3$ and $-2 \leq q \leq 3$.

- $\mathcal{J}(p, q)$ functions in the domain $p \geq 4, q \geq 0$

The recurrent relations in Appendix 9 give expressions for the finite part $J(p, q)$, the divergent part should be found by the procedure described in the beginning of this Section, see also (85).

It should be also noted that the formulas in Appendix 9 are valid only in the case $p>4$, in order to use them at $p=4$ the quantities $J(0, q)$ that appear in the right-hand part should be replaced by $B(0, q)$.

- $\mathcal{J}(p, q)$ functions in the domain $p \geq 4, q<0$ 
The explicit expressions for the finite parts $J(p, q)$ at $q=-1,-2$ and $p \leq 9$ are presented in the Appendix 10, the recurrent relations valid at $p>4, q<-2$ are given in the Appendix 11. To employ these relations at $p=4$, one should replace $J(0, q)$ that appears in the right-hand side by the function $B(0, q)$.

The divergent parts are given by the formula (87).

Therewith, it should be noted that the constants $X_{0} \div X_{3}$ that appear in some expressions for $J(p, q)$ at $p \leq 0$ cancel in the expressions for the integrals (51) at $p>0$ and thus their numerical values are not needed.

\subsection{Dimensional Regularization}

In the dimensional regularization, each integral $F(p, q ; n 1, n 2, n 3, n 4)$ (see (51)) is associated with the respective boson integral $B_{F}(p, q ; n 1, n 2, n 3, n 4)$ that has the same divergent part. $B_{F}(p, q ; n 1, n 2, n 3, n 4)$ is determined by the procedure similar to that indicated in subsection (3.1). For example, at $p>0, q \geq 2-p$

$$
B_{F}(p, q ; n 1, n 2, n 3, n 4)=\sum_{l=0}^{p+q-2} \frac{(-1)^{l}(p+l-1) !}{l !(p-1) !} \int \frac{d k}{(2 \pi)^{4}} \frac{\Delta^{l} \cos ^{n_{1}}\left(k_{1}\right) \ldots \cos ^{n_{4}}\left(k_{4}\right)}{\Delta_{B}^{p+q+l}} .
$$

Then we compute $B_{F}(p, q ; n 1, n 2, n 3, n 4)$ in the dimensional regularization as it is described in subsection (2.4) and $F(p, q ; n 1, n 2, n 3, n 4)-B_{F}(p, q ; n 1, n 2, n 3, n 4)$ (which is convergent) in the fictitious mass regularization. The sum of these quantities provides the sought for result.

\section{Conclusions}

The BCP algorithm described above and the explicit formulas obtained with it and presented in the Appendices make it a straightforward matter to express an integral of the type (51) at $p \leq 9$ and arbitrary values of $n_{1}, n_{2}, n_{3}, n_{4}$ and $q$ in terms of the constants $F_{0}, Z_{0}, Z_{1}$ and $Y_{0} \div Y_{11}$. In fact, these formulas provide a computer program, which can easily be realized with various packages. Such program was written in FORM and performed, some of the results are presented on the web: http://www.lattice.itep.ru/ pbaivid/lattpt/

These are

- the values of the functions $J(p, q)$ and $B(p, q)$ at $-26 \leq p \leq 0,-56-2 p \leq q \leq 34$ and the values of $J(p, q)$ at $1 \leq p \leq 9, \quad-28 \leq q \leq 33-p$;

- the expressions for the integrals of the type (51) at some particular values of $p$ and $q$ and $n_{1} \leq 6$

- the program for the computation of the integrals (51) at $0 \leq p, q \leq 9$ and $n_{\mu}^{\text {max }} \leq 6$ that can be readily used by anyone.

I hope that this work will facilitate using the BCP algorithm in practical computations.

Acknowledgments: I am grateful to H.Perlt, A.Schiller, and V. Bornyakov for stimulating discussions, to P.Buividovich for the help with the presentation on the web, and to the Leipzig University, where this study was started, for hospitality. This work was supported in part by the grant for scientific schools no. NSh-679.2008.2 and by the Russian Foundation for Basic Research (RFBR grant no. 07-02-0237). 


\section{Appendix 1.}

Some values used in the text are listed below.

The integrals defined in (111) at $4 \leq q \leq 12$ (see also (30)

$$
\begin{aligned}
& \mathcal{B}_{4}=917 / 2 ; \\
& \mathcal{B}_{5}=2514 ; \\
& \mathcal{B}_{6}=14376 ; \\
& \mathcal{B}_{7}=85152 ; \\
& \mathcal{B}_{8}=16628949 / 32 ; \\
& \mathcal{B}_{9}=26026877 / 8 ; \\
& \mathcal{B}_{10}=333148183 / 16 ; \\
& \mathcal{B}_{11}=543325293 / 4 ; \\
& \mathcal{B}_{12}=14415564199 / 16 ;
\end{aligned}
$$

The coefficients introduced in formula (13) are

$$
\begin{aligned}
& b_{0}=1 ; \\
& b_{1}=1 / 2 \text {; } \\
& b_{2}=3 / 8 \\
& b_{3}=13 / 32 \text {; } \\
& b_{4}=77 / 128 \\
& b_{5}=297 / 256 \\
& b_{6}=5727 / 2048 \text {; } \\
& b_{7}=66687 / 8192 \text {; } \\
& b_{8}=912303 / 32768 \text {; } \\
& b_{9}=3586545 / 32768 \text {; } \\
& b_{10}=127448505 / 262144 \text {; } \\
& b_{11}=2523924765 / 1048576 \text {; } \\
& b_{12}=110207056005 / 8388608 \text {; } \\
& b_{13}=657259273755 / 8388608 \text {; } \\
& b_{14}=68022530602425 / 134217728 \text {; } \\
& b_{15}=1897008475419225 / 536870912 \text {; } \\
& b_{16}=56719614296927925 / 2147483648 \text {; } \\
& b_{17}=226232753142332475 / 1073741824 \text {; } \\
& b_{18}=15346146376168947675 / 8589934592 \text {; } \\
& b_{19}=275641831899783381375 / 17179869184 \text {; } \\
& b_{20}=41819089838429396989125 / 274877906944 \text {; }
\end{aligned}
$$

The coefficients $c_{q}\left(n_{1}, n_{2}, n_{3}, n_{4}\right)$ used in the dimensional regularization are introduced in (399):

$$
\begin{aligned}
& c_{0}\left(n_{1}, n_{2}, n_{3}, n_{4}\right)=0, \\
& c_{1}\left(n_{1}, n_{2}, n_{3}, n_{4}\right)=1 / 8,
\end{aligned}
$$


these equations at $q=0,1$ are valid for all $n_{\mu}$. The coefficients $c_{q}=c_{q}(0,0,0,0)$ at $q \leq 10$ are

$$
\begin{aligned}
c_{2}(0,0,0,0) & =1 / 8 \\
c_{3}(0,0,0,0) & =55 / 384 \\
c_{4}(0,0,0,0) & =5 / 24 \\
c_{5}(0,0,0,0) & =1973 / 5120 \\
c_{6}(0,0,0,0) & =54583 / 61440 \\
c_{7}(0,0,0,0) & =8558131 / 3440640 \\
c_{8}(0,0,0,0) & =4727509 / 573440 \\
c_{9}(0,0,0,0) & =652905649 / 20643840 \\
c_{10}(0,0,0,0) & =2276619691 / 16515072
\end{aligned}
$$

And the values some other of the coefficients $c_{q}\left(n_{1}, n_{2}, n_{3}, n_{4}\right)$ :

$$
\begin{aligned}
& c_{2}(1,0,0,0)=1 / 16 ; \\
& c_{2}(1,1,0,0)=0 \text {; } \\
& c_{2}(1,1,1,0)=-1 / 16 \text {; } \\
& c_{2}(1,1,1,1)=-1 / 8 \text {; } \\
& c_{2}(2,0,0,0)=0 \text {; } \\
& c_{2}(2,1,0,0)=-1 / 16 \text {; } \\
& c_{2}(2,1,1,0)=-1 / 8 \text {; } \\
& c_{2}(2,1,1,1)=-3 / 16 \text {; } \\
& c_{3}(1,0,0,0)=25 / 384 \text {; } \\
& c_{3}(1,1,0,0)=7 / 384 \text {; } \\
& c_{3}(1,1,1,0)=1 / 384 \text {; } \\
& c_{4}(1,0,0,0)=27 / 256 ; \\
& c_{4}(1,1,0,0)=19 / 384 \text {; } \\
& c_{4}(1,1,1,0)=19 / 768 ; \\
& c_{4}(1,1,1,1)=1 / 64 \text {; } \\
& c_{4}(2,0,0,0)=55 / 384 \text {; } \\
& c_{5}(1,0,0,0)=1143 / 5120 ; \\
& c_{5}(1,1,0,0)=1999 / 15360 ; \\
& c_{5}(2,0,0,0)=1433 / 5120 ; \\
& c_{6}(1,0,0,0)=7085 / 12288 ; \\
& c_{6}(1,1,0,0)=23387 / 61440 ; \\
& c_{6}(2,0,0,0)=40867 / 61440 ;
\end{aligned}
$$




\section{Appendix 2.}

$$
\begin{aligned}
& L(0,2)=\left(l_{C}+1 / 2 l_{C}^{2}\right) /(2 \pi)^{2} ; \\
& L(0,3)=\left(-3 / 4 \mu_{B}^{-2}-1 / 2 \mu_{B}^{-2} l_{C}+5 / 8 l_{C}+1 / 8 l_{C}^{2}\right) /(2 \pi)^{2} ; \\
& L(0,4)=\left(\left(-1 / 6 \mu_{B}^{-4}-1 / 12 \mu_{B}^{-2}+137 / 960\right) l_{C}\right. \\
& \left.-5 / 36 \mu_{B}^{-4}-31 / 144 \mu_{B}^{-2}+1 / 32 l_{C}^{2}\right) /(2 \pi)^{2} ; \\
& L(0,5)=\left(\left(-1 / 12 \mu_{B}^{-6}-1 / 48 \mu_{B}^{-4}-1 / 64 \mu_{B}^{-2}+15527 / 322560\right) l_{C}\right. \\
& \left.-7 / 144 \mu_{B}^{-6}-101 / 2880 \mu_{B}^{-4}-151 / 3840 \mu_{B}^{-2}+13 / 1536 l_{C}^{2}\right) /(2 \pi)^{2} ; \\
& L(0,6)=\left(\left(-1 / 20 \mu_{B}^{-8}-1 / 120 \mu_{B}^{-6}-1 / 320 \mu_{B}^{-4}-13 / 3840 \mu_{B}^{-2}+172241 / 12902400\right) l_{C}\right. \\
& -9 / 400 \mu_{B}^{-8}-77 / 7200 \mu_{B}^{-6}-709 / 134400 \mu_{B}^{-4}-3953 / 403200 \mu_{B}^{-2} \\
& \left.+77 / 30720 l_{C}^{2}\right) /(2 \pi)^{2} \text {; } \\
& L(0,7)=\left(\left(-1 / 30 \mu_{B}^{-10}-1 / 240 \mu_{B}^{-8}-1 / 960 \mu_{B}^{-6}\right.\right. \\
& \left.-13 / 23040 \mu_{B}^{-4}-77 / 92160 \mu_{B}^{-2}+457867 / 94617600\right) l_{C} \\
& -11 / 900 \mu_{B}^{-10}-439 / 100800 \mu_{B}^{-8}-53 / 38400 \mu_{B}^{-6} \\
& \left.-5371 / 4838400 \mu_{B}^{-4}-183101 / 77414400 \mu_{B}^{-2}+33 / 40960 l_{C}^{2}\right) /(2 \pi)^{2} ; \\
& L(0,8)=\left(\left(-1 / 42 \mu_{B}^{-12}-1 / 420 \mu_{B}^{-10}-1 / 2240 \mu_{B}^{-8}-13 / 80640 \mu_{B}^{-6}\right.\right. \\
& \left.-11 / 92160 \mu_{B}^{-4}-33 / 143360 \mu_{B}^{-2}+51135377 / 31791513600\right) l_{C} \\
& -13 / 1764 \mu_{B}^{-12}-743 / 352800 \mu_{B}^{-10}-2789 / 5644800 \mu_{B}^{-8} \\
& -3371 / 13547520 \mu_{B}^{-6}-28441 / 121651200 \mu_{B}^{-4}-10159 / 14450688 \mu_{B}^{-2} \\
& \left.+1909 / 6881280 l_{C}^{2}\right) /(2 \pi)^{2} \text {; } \\
& D(0,2)=-l_{C} /(2 \pi)^{2} \\
& D(0,3)=\left(1 / 2 \mu_{B}^{-2}-1 / 4 l_{C}\right) /(2 \pi)^{2} ; \\
& D(0,4)=\left(1 / 6 \mu_{B}^{-4}+1 / 12 \mu_{B}^{-2}-1 / 16 l_{C}\right) /(2 \pi)^{2} ; \\
& D(0,5)=\left(1 / 12 \mu_{B}^{-6}+1 / 48 \mu_{B}^{-4}+1 / 64 \mu_{B}^{-2}-13 / 768 l_{C}\right) /(2 \pi)^{2} ; \\
& D(0,6)=\left(1 / 20 \mu_{B}^{-8}+1 / 120 \mu_{B}^{-6}+1 / 320 \mu_{B}^{-4}+13 / 3840 \mu_{B}^{-2}-77 / 15360 l_{C}\right) /(2 \pi)^{2} ; \\
& D(0,7)=\left(1 / 30 \mu_{B}^{-10}+1 / 240 \mu_{B}^{-8}+1 / 960 \mu_{B}^{-6}+13 / 23040 \mu_{B}^{-4}\right. \\
& \left.+77 / 92160 \mu_{B}^{-2}-33 / 20480 l_{C}\right) /(2 \pi)^{2} ; \\
& D(0,8)=\left(1 / 42 \mu_{B}^{-12}+1 / 420 \mu_{B}^{-10}+1 / 2240 \mu_{B}^{-8}+13 / 80640 \mu_{B}^{-6}\right. \\
& \left.+11 / 92160 \mu_{B}^{-4}+33 / 143360 \mu_{B}^{-2}-1909 / 3440640 l_{C}\right) /(2 \pi)^{2} \text {; } \\
& D(1,1)=\left(-l_{C}\right) /(2 \pi)^{2} \\
& D(1,2)=\left(1 / 2 \mu_{B}^{-2}\right) /(2 \pi)^{2} \text {; } \\
& D(1,3)=\left(1 / 6 \mu_{B}^{-4}+1 / 48 \mu_{B}^{-2}-11 / 160 l_{C}\right) /(2 \pi)^{2} ; \\
& D(1,4)=\left(1 / 12 \mu_{B}^{-6}+1 / 120 \mu_{B}^{-4}+7 / 480 \mu_{B}^{-2}+1 / 1680 l_{C}\right) /(2 \pi)^{2} ; \\
& D(1,5)=\left(1 / 20 \mu_{B}^{-8}+1 / 240 \mu_{B}^{-6}+3 / 1120 \mu_{B}^{-4}+19 / 21504 \mu_{B}^{-2}-1339 / 215040 l_{C}\right) /(2 \pi)^{2} ; \\
& D(1,6)=\left(1 / 30 \mu_{B}^{-10}+1 / 420 \mu_{B}^{-8}+23 / 26880 \mu_{B}^{-6}+13 / 53760 \mu_{B}^{-4}\right. \\
& \left.+181 / 215040 \mu_{B}^{-2}-67 / 630784 l_{C}\right) /(2 \pi)^{2} \text {; } \\
& D(1,7)=\left(1 / 42 \mu_{B}^{-12}+1 / 672 \mu_{B}^{-10}+29 / 80640 \mu_{B}^{-8}+277 / 3225600 \mu_{B}^{-6}\right.
\end{aligned}
$$


$\left.+15163 / 141926400 \mu_{B}^{-4}+46523 / 567705600 \mu_{B}^{-2}-52001 / 75694080 l_{C}\right) /(2 \pi)^{2} ;$

$D(2,1)=\left(1 / 2 \mu_{B}^{-2}+1 / 4 l_{C}\right) /(2 \pi)^{2} ;$

$D(2,2)=\left(1 / 6 \mu_{B}^{-4}-1 / 24 \mu_{B}^{-2}-23 / 160 l_{C}\right) /(2 \pi)^{2} ;$

$D(2,3)=\left(1 / 12 \mu_{B}^{-6}-1 / 240 \mu_{B}^{-4}+1 / 40 \mu_{B}^{-2}+1093 / 26880 l_{C}\right) /(2 \pi)^{2} ;$

$D(2,4)=\left(1 / 20 \mu_{B}^{-8}+13 / 3360 \mu_{B}^{-4}-37 / 8960 \mu_{B}^{-2}-4141 / 215040 l_{C}\right) /(2 \pi)^{2} ;$

$D(2,5)=\left(1 / 30 \mu_{B}^{-10}+1 / 1680 \mu_{B}^{-8}+29 / 26880 \mu_{B}^{-6}-1 / 3072 \mu_{B}^{-4}\right.$

$\left.+31 / 15360 \mu_{B}^{-2}+34689 / 6307840 l_{C}\right) /(2 \pi)^{2}$;

$D(2,6)=\left(1 / 42 \mu_{B}^{-12}+1 / 1680 \mu_{B}^{-10}+11 / 26880 \mu_{B}^{-8}-17 / 537600 \mu_{B}^{-6}\right.$

$\left.+4747 / 23654400 \mu_{B}^{-4}-36473 / 94617600 \mu_{B}^{-2}-128103 / 50462720 l_{C}\right) /(2 \pi)^{2}$;

$D(3,1)=\left(1 / 6 \mu_{B}^{-4}-5 / 48 \mu_{B}^{-2}-23 / 80 l_{C}\right) /(2 \pi)^{2} ;$

$D(3,2)=\left(1 / 12 \mu_{B}^{-6}-1 / 60 \mu_{B}^{-4}+3 / 64 \mu_{B}^{-2}+331 / 2688 l_{C}\right) /(2 \pi)^{2} ;$

$D(3,3)=\left(1 / 20 \mu_{B}^{-8}-1 / 240 \mu_{B}^{-6}+3 / 448 \mu_{B}^{-4}-19 / 1344 \mu_{B}^{-2}-12241 / 215040 l_{C}\right) /(2 \pi)^{2}$;

$D(3,4)=\left(1 / 30 \mu_{B}^{-10}-1 / 840 \mu_{B}^{-8}+23 / 13440 \mu_{B}^{-6}-19 / 13440 \mu_{B}^{-4}\right.$

$\left.+481 / 86016 \mu_{B}^{-2}+35879 / 1576960 l_{C}\right) /(2 \pi)^{2} ;$

$D(3,5)=\left(1 / 42 \mu_{B}^{-12}-1 / 3360 \mu_{B}^{-10}+1 / 1680 \mu_{B}^{-8}-53 / 215040 \mu_{B}^{-6}\right.$ $\left.+4801 / 9461760 \mu_{B}^{-4}-33797 / 18923520 \mu_{B}^{-2}-492689 / 50462720 l_{C}\right) /(2 \pi)^{2}$;

$D(4,1)=\left(1 / 12 \mu_{B}^{-6}-7 / 240 \mu_{B}^{-4}+77 / 960 \mu_{B}^{-2}+7201 / 26880 l_{C}\right) /(2 \pi)^{2} ;$

$D(4,2)=\left(1 / 20 \mu_{B}^{-8}-1 / 120 \mu_{B}^{-6}+5 / 448 \mu_{B}^{-4}-85 / 2688 \mu_{B}^{-2}-29671 / 215040 l_{C}\right) /(2 \pi)^{2}$;

$D(4,3)=\left(1 / 30 \mu_{B}^{-10}-1 / 336 \mu_{B}^{-8}+37 / 13440 \mu_{B}^{-6}-19 / 5760 \mu_{B}^{-4}\right.$

$\left.+17267 / 1290240 \mu_{B}^{-2}+51223 / 788480 l_{C}\right) /(2 \pi)^{2} ;$

$D(4,4)=\left(1 / 42 \mu_{B}^{-12}-1 / 840 \mu_{B}^{-10}+37 / 40320 \mu_{B}^{-8}-11 / 17920 \mu_{B}^{-6}\right.$ $\left.+16853 / 14192640 \mu_{B}^{-4}-923 / 177408 \mu_{B}^{-2}-2284033 / 75694080 l_{C}\right) /(2 \pi)^{2} ;$

$D(5,1)=\left(1 / 20 \mu_{B}^{-8}-1 / 80 \mu_{B}^{-6}+29 / 1680 \mu_{B}^{-4}\right.$

$\left.-2117 / 35840 \mu_{B}^{-2}-30869 / 107520 l_{C}\right) /(2 \pi)^{2}$;

$D(5,2)=\left(1 / 30 \mu_{B}^{-10}-1 / 210 \mu_{B}^{-8}+113 / 26880 \mu_{B}^{-6}-1009 / 161280 \mu_{B}^{-4}\right.$ $\left.+35873 / 1290240 \mu_{B}^{-2}+482633 / 3153920 l_{C}\right) /(2 \pi)^{2} ;$

$D(5,3)=\left(1 / 42 \mu_{B}^{-12}-1 / 480 \mu_{B}^{-10}+37 / 26880 \mu_{B}^{-8}-767 / 645120 \mu_{B}^{-6}\right.$ $\left.+69689 / 28385280 \mu_{B}^{-4}-116749 / 9461760 \mu_{B}^{-2}-5912603 / 75694080 l_{C}\right) /(2 \pi)^{2}$;

$D(6,1)=\left(1 / 30 \mu_{B}^{-10}-11 / 1680 \mu_{B}^{-8}+163 / 26880 \mu_{B}^{-6}\right.$ $\left.-3407 / 322560 \mu_{B}^{-4}+66911 / 1290240 \mu_{B}^{-2}+400193 / 1261568 l_{C}\right) /(2 \pi)^{2} ;$

$D(6,2)=\left(1 / 42 \mu_{B}^{-12}-1 / 336 \mu_{B}^{-10}+53 / 26880 \mu_{B}^{-8}-467 / 230400 \mu_{B}^{-6}\right.$ $\left.+162457 / 35481600 \mu_{B}^{-4}-1214401 / 47308800 \mu_{B}^{-2}-26852377 / 151388160 l_{C}\right) /(2 \pi)^{2} ;$

$D(7,1)=\left(1 / 42 \mu_{B}^{-12}-13 / 3360 \mu_{B}^{-10}+109 / 40320 \mu_{B}^{-8}-10267 / 3225600 \mu_{B}^{-6}\right.$ $\left.+53257 / 6758400 \mu_{B}^{-4}-27585673 / 567705600 \mu_{B}^{-2}-18404583 / 50462720 l_{C}\right) /(2 \pi)^{2}$;

$$
\begin{aligned}
& D(2,0)=\left(-l_{C}\right) /(2 \pi)^{2} \\
& D(3,0)=\left(1 / 2 \mu_{B}^{-2}+1 / 2 l_{C}\right) /(2 \pi)^{2}
\end{aligned}
$$




$$
\begin{aligned}
& D(3,-1)=\left(-l_{C}\right) /(2 \pi)^{2} ; \\
& D(4,0)=\left(1 / 6 \mu_{B}^{-4}-1 / 6 \mu_{B}^{-2}-1 / 2 l_{C}\right) /(2 \pi)^{2} ; \\
& D(4,-1)=\left(1 / 2 \mu_{B}^{-2}+3 / 4 l_{C}\right) /(2 \pi)^{2} \text {; } \\
& D(4,-2)=\left(-l_{C}\right) /(2 \pi)^{2} \text {; } \\
& D(5,0)=\left(1 / 12 \mu_{B}^{-6}-1 / 24 \mu_{B}^{-4}+1 / 8 \mu_{B}^{-2}+95 / 192 l_{C}\right) /(2 \pi)^{2} \text {; } \\
& D(5,-1)=\left(1 / 6 \mu_{B}^{-4}-11 / 48 \mu_{B}^{-2}-25 / 32 l_{C}\right) /(2 \pi)^{2} \text {; } \\
& D(5,-2)=\left(1 / 2 \mu_{B}^{-2}+l_{C}\right) /(2 \pi)^{2} \text {; } \\
& D(5,-3)=\left(-l_{C}\right) /(2 \pi)^{2} \text {; } \\
& D(6,0)=\left(1 / 20 \mu_{B}^{-8}-1 / 60 \mu_{B}^{-6}+1 / 40 \mu_{B}^{-4}-19 / 192 \mu_{B}^{-2}-1027 / 1920 l_{C}\right) /(2 \pi)^{2} \text {; } \\
& D(6,-1)=\left(1 / 12 \mu_{B}^{-6}-13 / 240 \mu_{B}^{-4}+29 / 160 \mu_{B}^{-2}+3163 / 3840 l_{C}\right) /(2 \pi)^{2} \text {; } \\
& D(6,-2)=\left(1 / 6 \mu_{B}^{-4}-7 / 24 \mu_{B}^{-2}-181 / 160 l_{C}\right) /(2 \pi)^{2} \text {; } \\
& D(6,-3)=\left(1 / 2 \mu_{B}^{-2}+5 / 4 l_{C}\right) /(2 \pi)^{2} \text {; } \\
& D(6,-4)=\left(-l_{C}\right) /(2 \pi)^{2} \text {; } \\
& D(7,0)=\left(1 / 30 \mu_{B}^{-10}-1 / 120 \mu_{B}^{-8}+1 / 120 \mu_{B}^{-6}-19 / 1152 \mu_{B}^{-4}\right. \\
& \left.+1027 / 11520 \mu_{B}^{-2}+3067 / 5120 l_{C}\right) /(2 \pi)^{2} \text {; } \\
& D(7,-1)=\left(1 / 20 \mu_{B}^{-8}-1 / 48 \mu_{B}^{-6}+11 / 320 \mu_{B}^{-4}-1181 / 7680 \mu_{B}^{-2}-14099 / 15360 l_{C}\right) /(2 \pi)^{2} \text {; } \\
& D(7,-2)=\left(1 / 12 \mu_{B}^{-6}-1 / 15 \mu_{B}^{-4}+239 / 960 \mu_{B}^{-2}+2447 / 1920 l_{C}\right) /(2 \pi)^{2} ; \\
& D(7,-3)=\left(1 / 6 \mu_{B}^{-4}-17 / 48 \mu_{B}^{-2}-31 / 20 l_{C}\right) /(2 \pi)^{2} \text {; } \\
& D(7,-4)=\left(1 / 2 \mu_{B}^{-2}+3 / 2 l_{C}\right) /(2 \pi)^{2} \text {; } \\
& D(7,-5)=\left(-l_{C}\right) /(2 \pi)^{2} \text {; } \\
& D(8,0)=\left(1 / 42 \mu_{B}^{-12}-1 / 210 \mu_{B}^{-10}+1 / 280 \mu_{B}^{-8}-19 / 4032 \mu_{B}^{-6}\right. \\
& \left.+1027 / 80640 \mu_{B}^{-4}-3067 / 35840 \mu_{B}^{-2}-74609 / 107520 l_{C}\right) /(2 \pi)^{2} \text {; } \\
& D(8,-1)=\left(1 / 30 \mu_{B}^{-10}-17 / 1680 \mu_{B}^{-8}+37 / 3360 \mu_{B}^{-6}\right. \\
& \left.-3923 / 161280 \mu_{B}^{-4}+9281 / 64512 \mu_{B}^{-2}+150949 / 143360 l_{C}\right) /(2 \pi)^{2} \text {; } \\
& D(8,-2)=\left(1 / 20 \mu_{B}^{-8}-1 / 40 \mu_{B}^{-6}+61 / 1344 \mu_{B}^{-4}-405 / 1792 \mu_{B}^{-2}-79489 / 53760 l_{C}\right) /(2 \pi)^{2} \text {; } \\
& D(8,-3)=\left(1 / 12 \mu_{B}^{-6}-19 / 240 \mu_{B}^{-4}+21 / 64 \mu_{B}^{-2}+10037 / 5376 l_{C}\right) /(2 \pi)^{2} \text {; } \\
& D(8,-4)=\left(1 / 6 \mu_{B}^{-4}-5 / 12 \mu_{B}^{-2}-163 / 80 l_{C}\right) /(2 \pi)^{2} \text {; } \\
& D(8,-5)=\left(1 / 2 \mu_{B}^{-2}+7 / 4 l_{C}\right) /(2 \pi)^{2} \text {; } \\
& D(8,-6)=\left(-l_{C}\right) /(2 \pi)^{2} \text {; } \\
& D(9,-1)=\left(1 / 42 \mu_{B}^{-12}-19 / 3360 \mu_{B}^{-10}+41 / 8960 \mu_{B}^{-8}-4303 / 645120 \mu_{B}^{-6}\right. \\
& \left.+50513 / 2580480 \mu_{B}^{-4}-163217 / 1146880 \mu_{B}^{-2}-8535263 / 6881280 l_{C}\right) /(2 \pi)^{2} \text {; } \\
& D(9,-2)=\left(1 / 30 \mu_{B}^{-10}-1 / 84 \mu_{B}^{-8}+379 / 26880 \mu_{B}^{-6}\right. \\
& \left.-791 / 23040 \mu_{B}^{-4}+5087 / 23040 \mu_{B}^{-2}+501267 / 286720 l_{C}\right) /(2 \pi)^{2} \text {; } \\
& D(9,-3)=\left(1 / 20 \mu_{B}^{-8}-7 / 240 \mu_{B}^{-6}+13 / 224 \mu_{B}^{-4}-6841 / 21504 \mu_{B}^{-2}-487141 / 215040 l_{C}\right) /(2 \pi)^{2} ; \\
& D(9,-4)=\left(1 / 12 \mu_{B}^{-6}-11 / 120 \mu_{B}^{-4}+67 / 160 \mu_{B}^{-2}+8807 / 3360 l_{C}\right) /(2 \pi)^{2} \text {; } \\
& D(9,-5)=\left(1 / 6 \mu_{B}^{-4}-23 / 48 \mu_{B}^{-2}-83 / 32 l_{C}\right) /(2 \pi)^{2} \text {; } \\
& D(9,-6)=\left(1 / 2 \mu_{B}^{-2}+2 l_{C}\right) /(2 \pi)^{2} \text {; } \\
& D(9,-7)=\left(-l_{C}\right) /(2 \pi)^{2} \text {; }
\end{aligned}
$$




\section{Appendix 3 .}

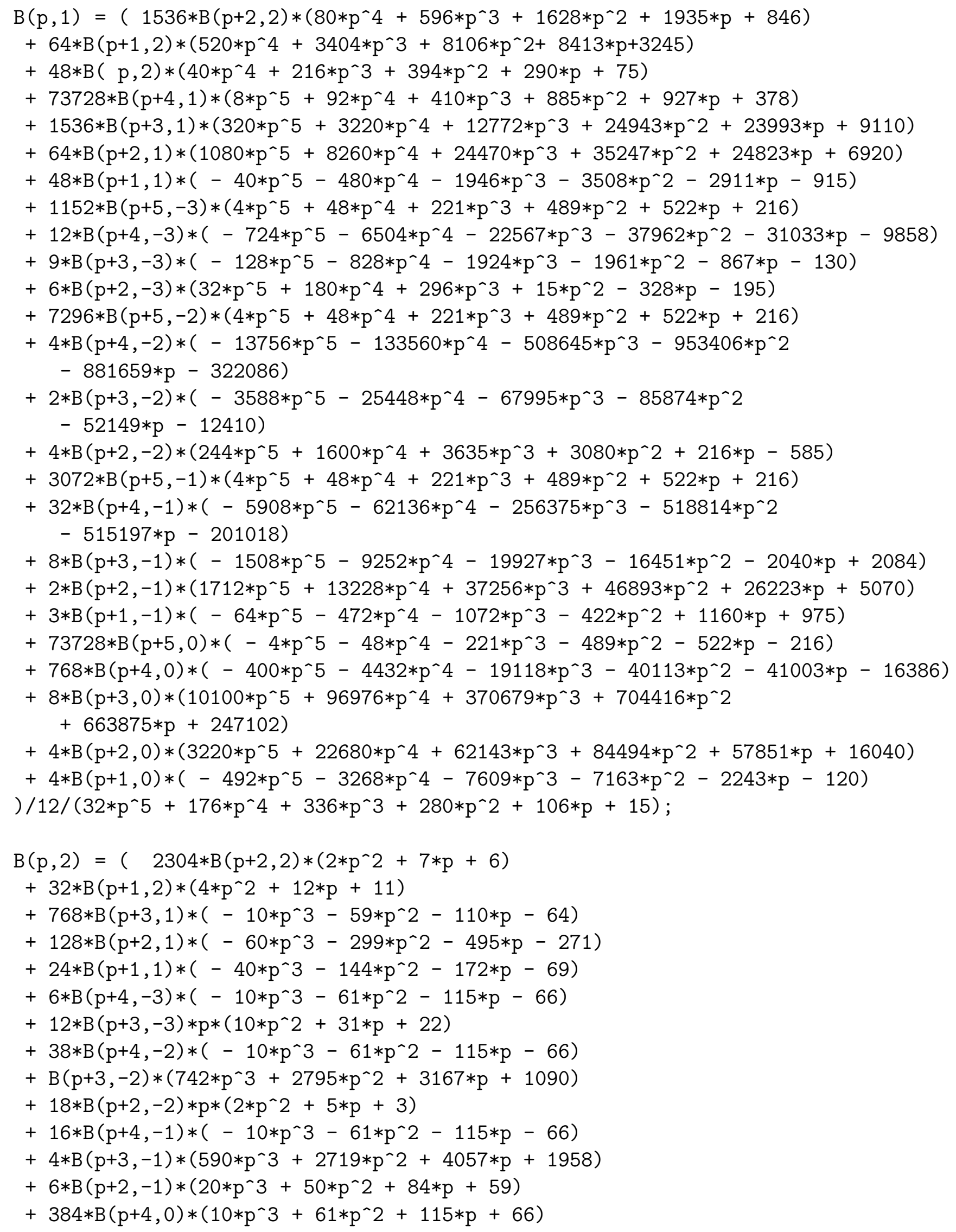




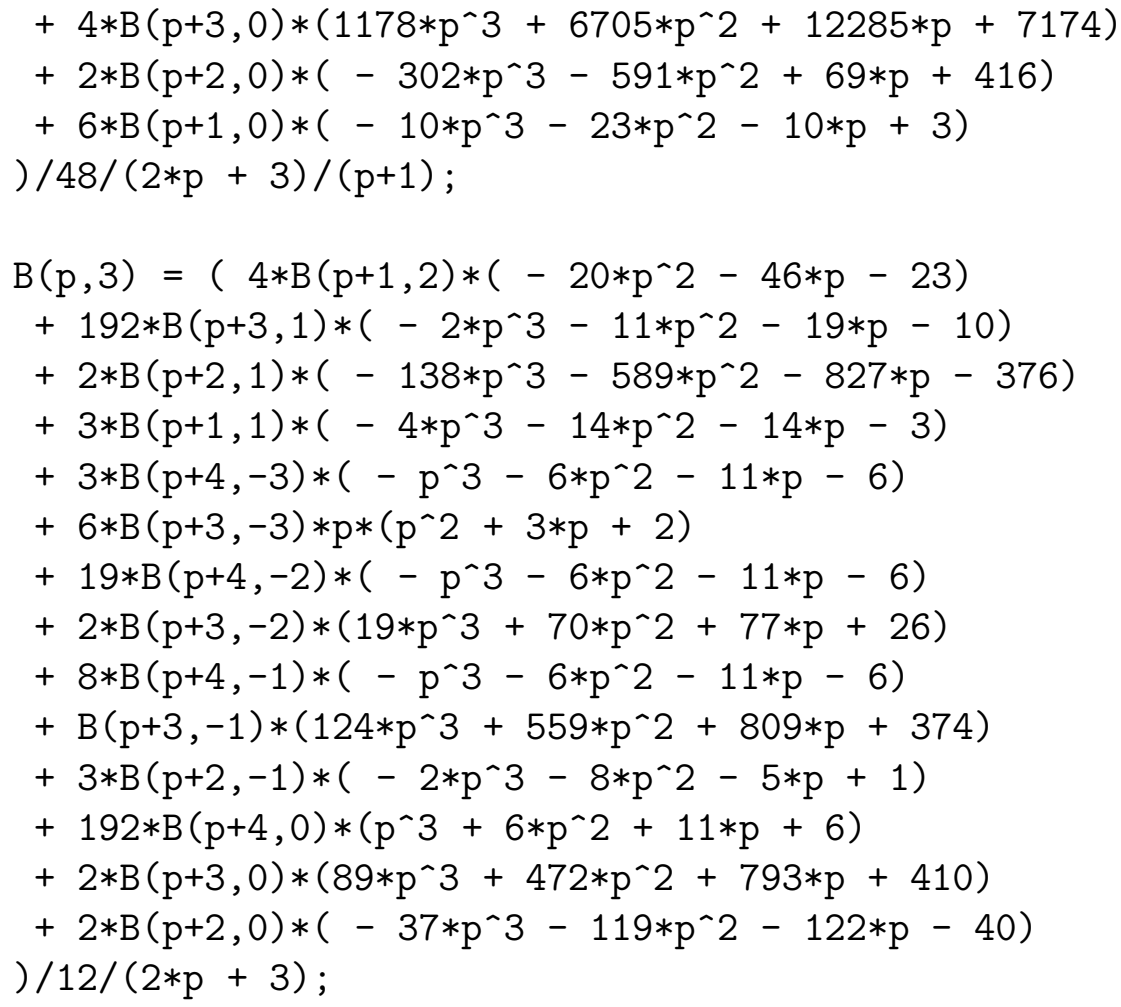

\section{Appendix 4.}

Here and below, the symbol $\mathrm{BB}(\mathrm{p}, \mathrm{q})$ in any Appendix designates $\mathcal{B}(p, q)$, whereas $\mathrm{B}(\mathrm{p}, \mathrm{q})$ designates $B(p, q)$.

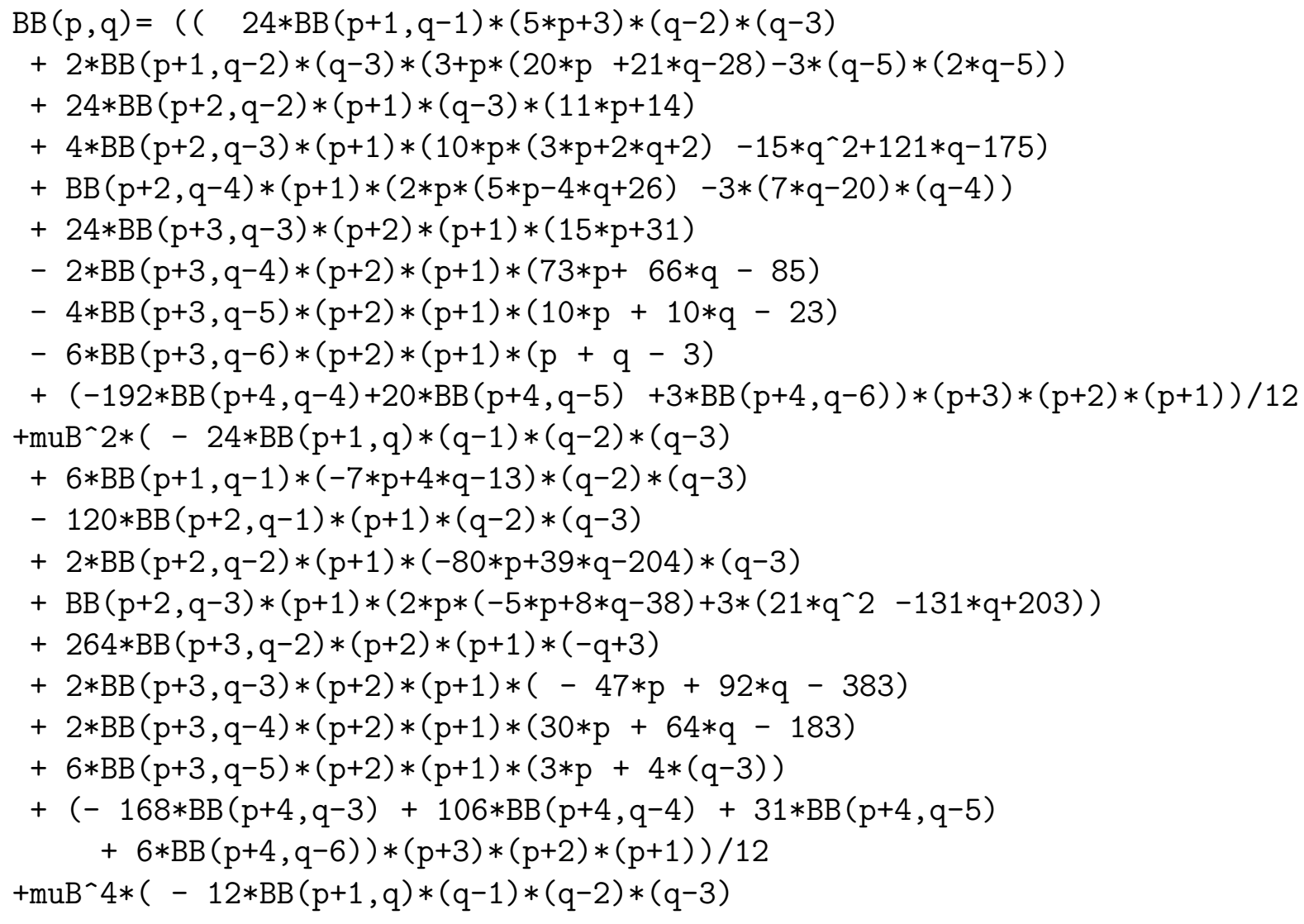




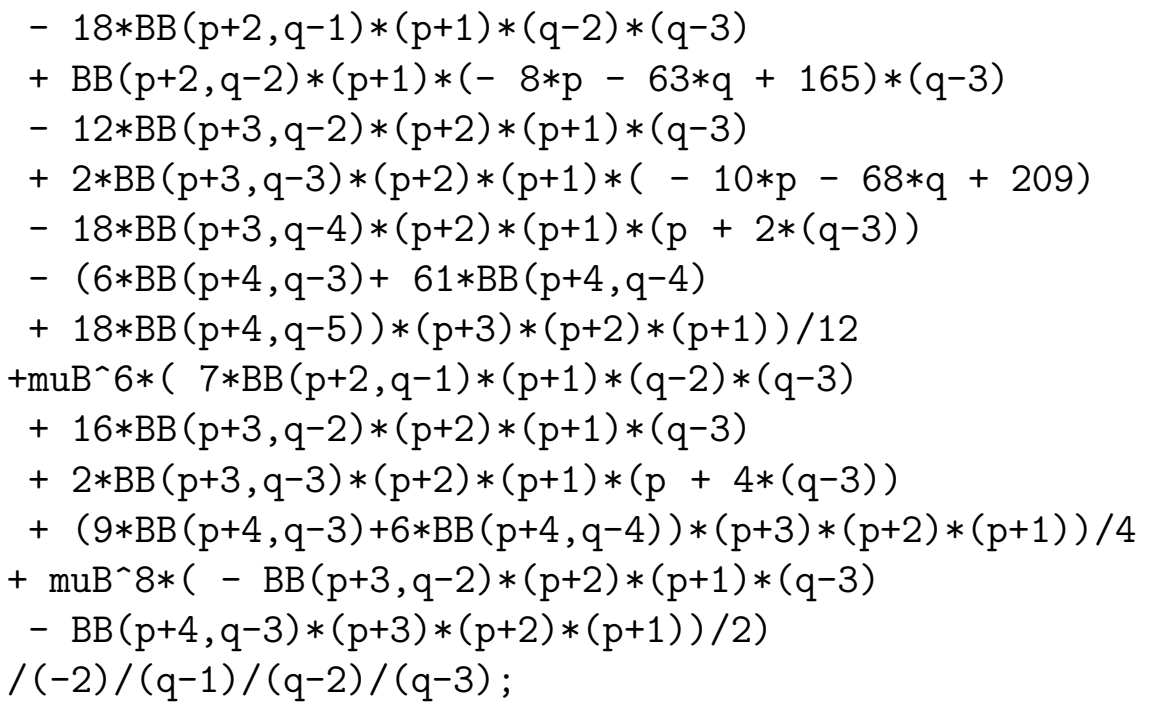

\section{Appendix 5.}

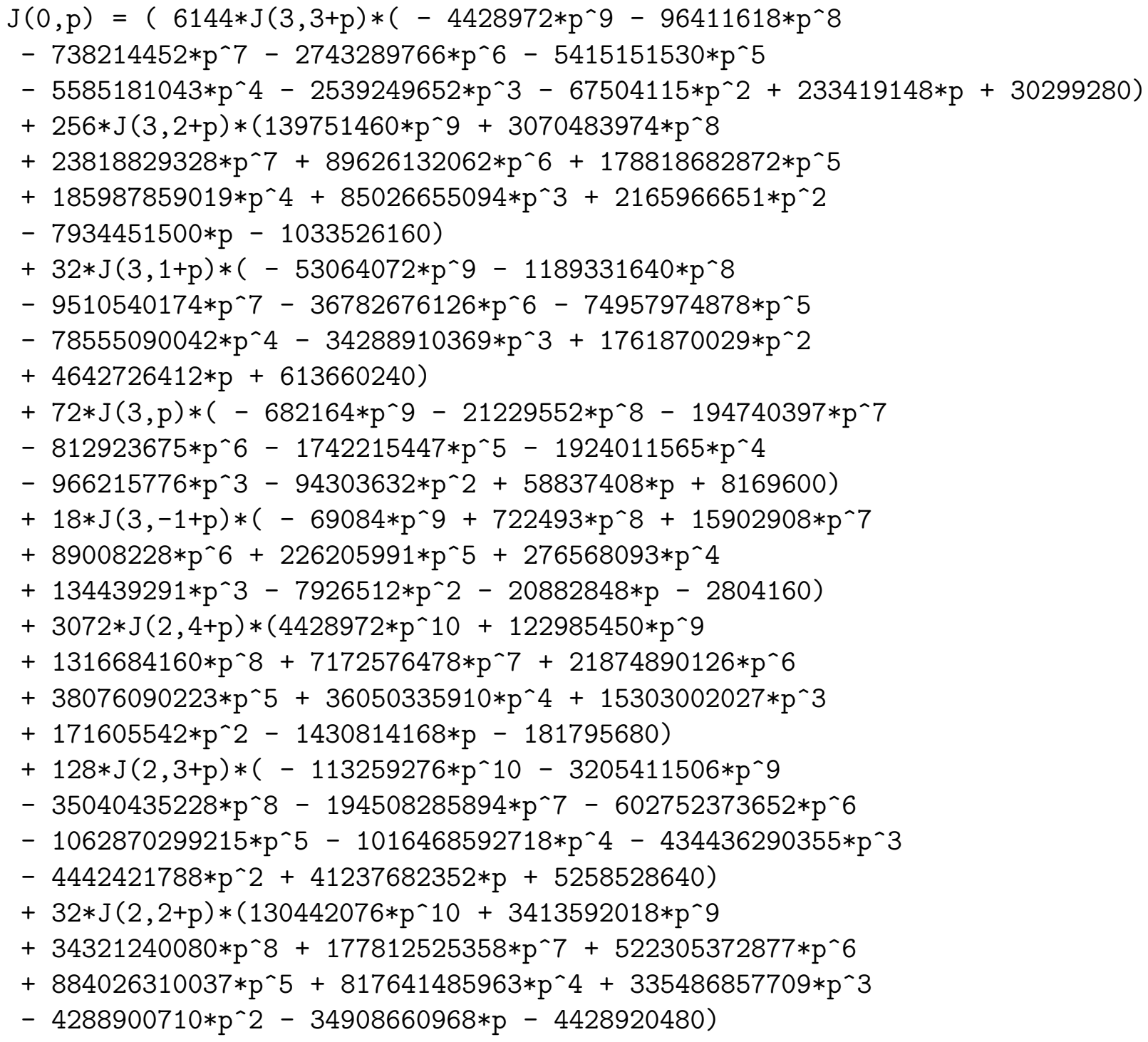




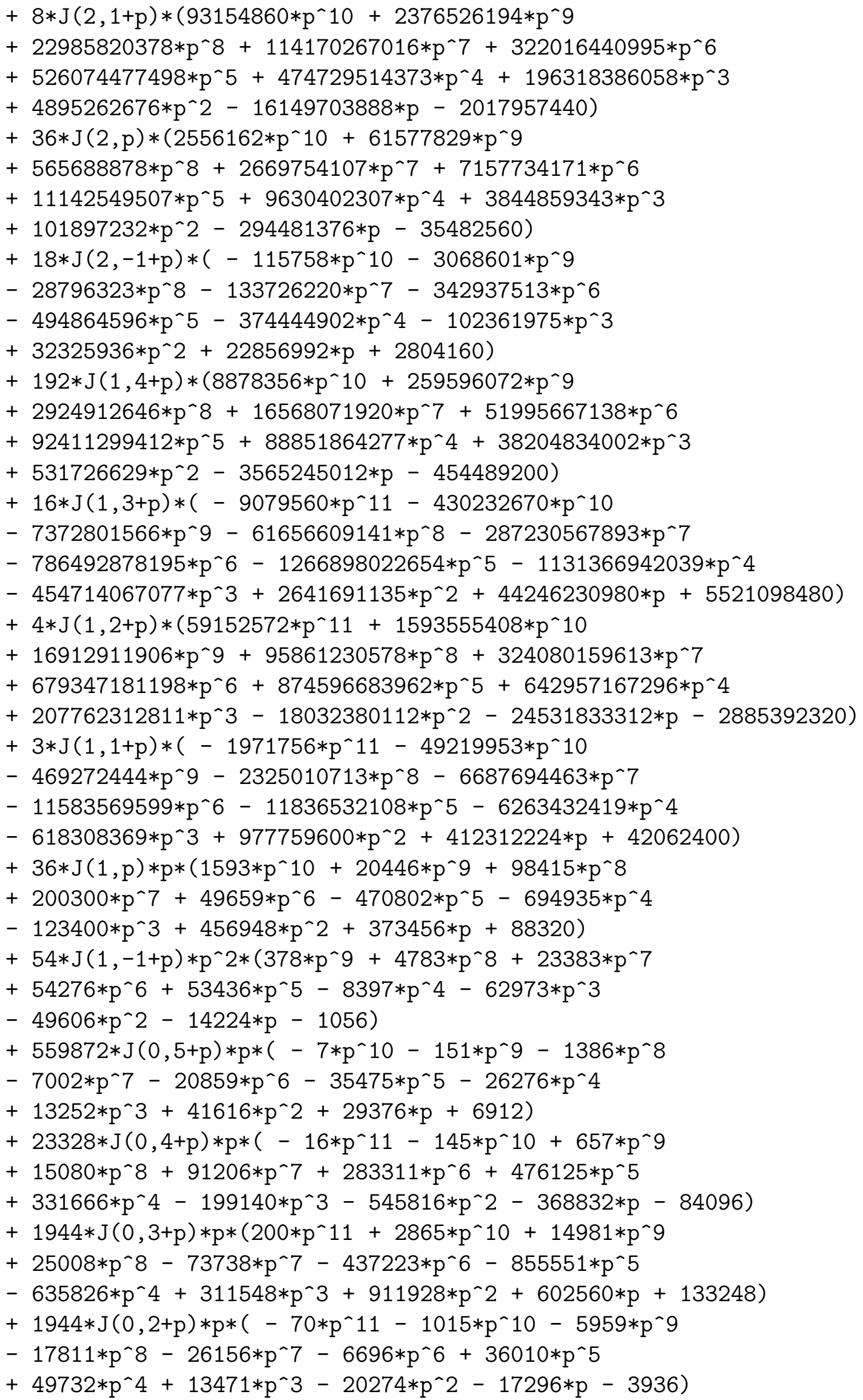




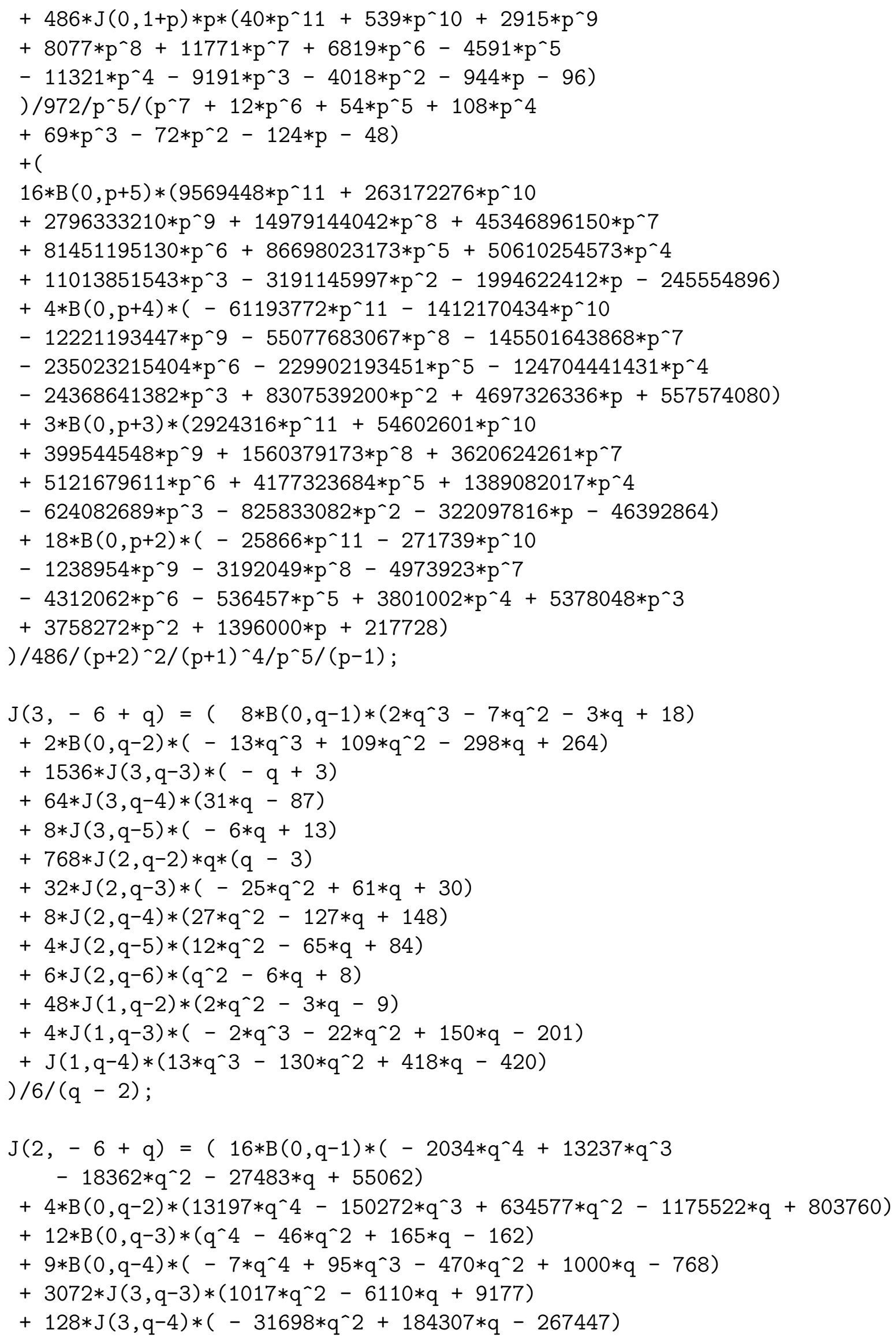




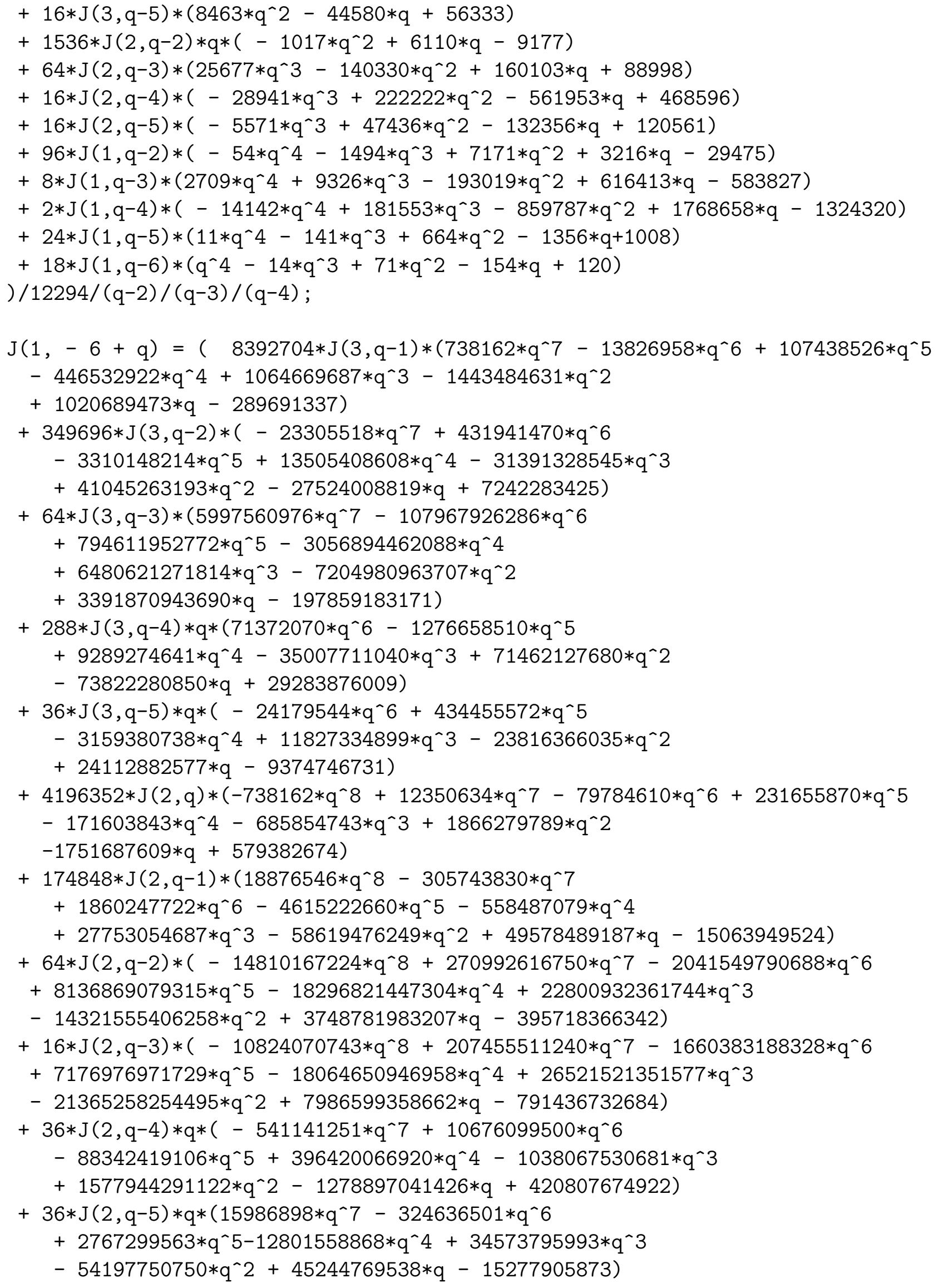


$+262272 * \mathrm{~J}(1, \mathrm{q}) *\left(-1476324 * \mathrm{q}^{\wedge} 8+22486782 * \mathrm{q}^{\wedge} 7\right.$

- $118088346 * q^{\wedge} 6+140996162 * q^{\wedge} 5+996391080 * q^{\wedge} 4$

- 4565718547*q^3 + 8063013471*q^2 - 6565443637*q + 2027839359)

$+21856 * \mathrm{~J}(1, \mathrm{q}-1) *\left(1479240 * \mathrm{q}^{\wedge} 9-718824 * \mathrm{q}^{\wedge} 8-290505888 * \mathrm{q}^{\wedge} 7\right.$

$+3040697422 * q^{\wedge} 6-14276034470 * q^{\wedge} 5+36530607805 * q^{\wedge} 4$

$\left.-52418121878 * q^{\wedge} 3+40019451544 * q^{\wedge} 2-13989602036 * q+1448456685\right)$

$+8 * \mathrm{~J}\left(1, \mathrm{q}^{-2}\right) * \mathrm{q} *\left(-6672113604 * \mathrm{q}^{\wedge} 8+143144560506 * \mathrm{q}^{\wedge} 7-1317848051532 * \mathrm{q}^{\wedge} 6\right.$

$+6794557026194 * \mathrm{q}^{\wedge} 5-21434683657627 * \mathrm{q}^{\wedge} 4+42303073328518 * \mathrm{q}^{\wedge} 3$

- 50886066609614*q^2 + 33974478049038*q - 9569982531879)

$+6 * \mathrm{~J}(1, \mathrm{q}-3) * \mathrm{q} *\left(221313528 * \mathrm{q}^{\wedge} 8-5165620026 * \mathrm{q}^{\wedge} 7+51969200474 * \mathrm{q}^{\wedge} 6\right.$

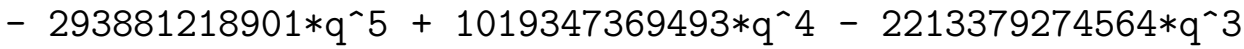

$\left.+2923819741196 * q^{\wedge} 2-2132296331629 * q+649364820429\right)$

$+27 * \mathrm{~J}\left(1, \mathrm{q}^{-4}\right) * \mathrm{q} *\left(-91046 * \mathrm{q}^{\wedge} 8+2191855 * \mathrm{q}^{\wedge} 7-22772339 * \mathrm{q}^{\wedge} 6\right.$

$+133314169 * q^{\wedge} 5-480172079 * q^{\wedge} 4+1085700520 * q^{\wedge} 3$

$\left.-1495620096 * \mathrm{q}^{\wedge} 2+1136307096 * \mathrm{q}-358858080\right)$

$+324 * \mathrm{~J}(1, \mathrm{q}-5) * \mathrm{q} *\left(-3304 * \mathrm{q}^{\wedge} 8+86533 * \mathrm{q}^{\wedge} 7-972784 * \mathrm{q}^{\wedge} 6\right.$

$+6115624 * q^{\wedge} 5-23438146 * q^{\wedge} 4+55819747 * q^{\wedge} 3$

$\left.-80159286 * q^{\wedge} 2+62865696 * q-20314080\right)$

$+63732096 * \mathrm{~J}(0, \mathrm{q}) * \mathrm{q}^{*}\left(-\mathrm{q}^{\wedge} 9+23 * \mathrm{q}^{\wedge} 8-228 * \mathrm{q}^{\wedge} 7\right.$

$+1278 * q^{\wedge} 6-4461 * q^{\wedge} 5+10047 * q^{\wedge} 4-14582 * q^{\wedge} 3$

$\left.+13132 * q^{\wedge} 2-6648 * q+1440\right)$

$+2655504 * \mathrm{~J}\left(0, \mathrm{q}^{-1}\right) * \mathrm{q} *\left(25 * \mathrm{q}^{\wedge} 9-625 * \mathrm{q}^{\wedge} 8+6812 * \mathrm{q}^{\wedge} 7\right.$

$-42490 * q^{\wedge} 6+167117 * q^{\wedge} 5-429505 * q^{\wedge} 4+720358 * q^{\wedge} 3$

$\left.-758580 * q^{\wedge} 2+453528 * q-116640\right)$

$+663876 * \mathrm{~J}\left(0, \mathrm{q}^{-2}\right) * \mathrm{q} *\left(-35 * \mathrm{q}^{\wedge} 9+945 * \mathrm{q}^{\wedge} 8-11182 * \mathrm{q}^{\wedge} 7\right.$

$+76062 * q^{\wedge} 6-327403 * q^{\wedge} 5+923145 * q^{\wedge} 4-1700348 * q^{\wedge} 3+1964808 * q^{\wedge} 2$

$-1284552 * \mathrm{q}+358560)$

$+663876 * \mathrm{~J}\left(0, \mathrm{q}^{-3}\right) * \mathrm{q} *\left(5 * \mathrm{q}^{\wedge} 9-145 * \mathrm{q}^{\wedge} 8+1845 * \mathrm{q}^{\wedge} 7-13500 * \mathrm{q}^{\wedge} 6\right.$

$\left.+62476 * q^{\wedge} 5-189120 * q^{\wedge} 4+373040 * q^{\wedge} 3-459875 * q^{\wedge} 2+318994 * q-93720\right)$

$+165969 * \mathrm{~J}\left(0, \mathrm{q}^{-4}\right) * \mathrm{q}^{*}\left(-\mathrm{q}^{\wedge} 9+31 * \mathrm{q}^{\wedge} 8-421 * \mathrm{q}^{\wedge} 7+3281 * \mathrm{q}^{\wedge} 6-16130 * \mathrm{q}^{\wedge} 5\right.$

$\left.+51704 * q^{\wedge} 4-107584 * q^{\wedge} 3+139264 * q^{\wedge} 2-100864 * q+30720\right)$

)$/ 25515 / \mathrm{q} /(\mathrm{q}-1) /(\mathrm{q}-2) /(\mathrm{q}-3) /(\mathrm{q}-4) /(\mathrm{q}-4) /(\mathrm{q}-5) /(\mathrm{q}-5) /(7 * \mathrm{q}-24)$

$+\left(87424 * B\left(0, q^{1}\right) *\left(-1476324 * q^{\wedge} 6+16581486 * q^{\wedge} 5-47333430 * q^{\wedge} 4\right.\right.$

$\left.-98082016 * \mathrm{q}^{\wedge} 3+746063306 * \mathrm{q}^{\wedge} 2-1287219275 * \mathrm{q}+675946453\right)$

$+43712 * \mathrm{~B}(0, \mathrm{q}) *\left(4862637 * \mathrm{q}^{\wedge} 6-78129165 * \mathrm{q}^{\wedge} 5+502175106 * \mathrm{q}^{\wedge} 4\right.$

$\left.-1641458936 * q^{\wedge} 3+2853167747 * q^{\wedge} 2-2474965235 * q+830365446\right)$

$+24 * B\left(0, q^{-1}\right) *\left(-187645068 * q^{\wedge} 6+3303988746 * q^{\wedge} 5-23822963042 * q^{\wedge} 4\right.$

$\left.+90075021407 * q^{\wedge} 3-188498675784 * q^{\wedge} 2+207202955323 * q-93562325614\right)$

$+108 * \mathrm{~B}\left(0, \mathrm{q}^{-2}\right) *\left(-2349349 * \mathrm{q}^{\wedge} 6+47038978 * \mathrm{q}^{\wedge} 5-386433111 * \mathrm{q}^{\wedge} 4\right.$

$\left.+1668058574 * q^{\wedge} 3-3990763652 * q^{\wedge} 2+5015869656 * q-2585112480\right)$

$+324 * \mathrm{~B}\left(0, \mathrm{q}^{-3}\right) *\left(125620 * \mathrm{q}^{\wedge} 6-2697750 * \mathrm{q}^{\wedge} 5+23873044 * \mathrm{q}^{\wedge} 4\right.$

$\left.-111347697 * q^{\wedge} 3+288317245 * q^{\wedge} 2-392099154 * q^{2}+218030520\right)$

$+1701 * \mathrm{~B}\left(0, \mathrm{q}^{-4}\right) *\left(-631 * \mathrm{q}^{\wedge} 6+14513 * \mathrm{q}^{\wedge} 5-137798 * \mathrm{q}^{\wedge} 4\right.$

$\left.+690128 * q^{-} 3-1917728 * q^{\wedge} 2+2792192 * q-1653760\right)$

)$/ 51030 /(\mathrm{q}-2) /(\mathrm{q}-4) /(\mathrm{q}-4) /(\mathrm{q}-5) /(\mathrm{q}-5) /(7 * \mathrm{q}-24)$; 


\section{Appendix 6.}

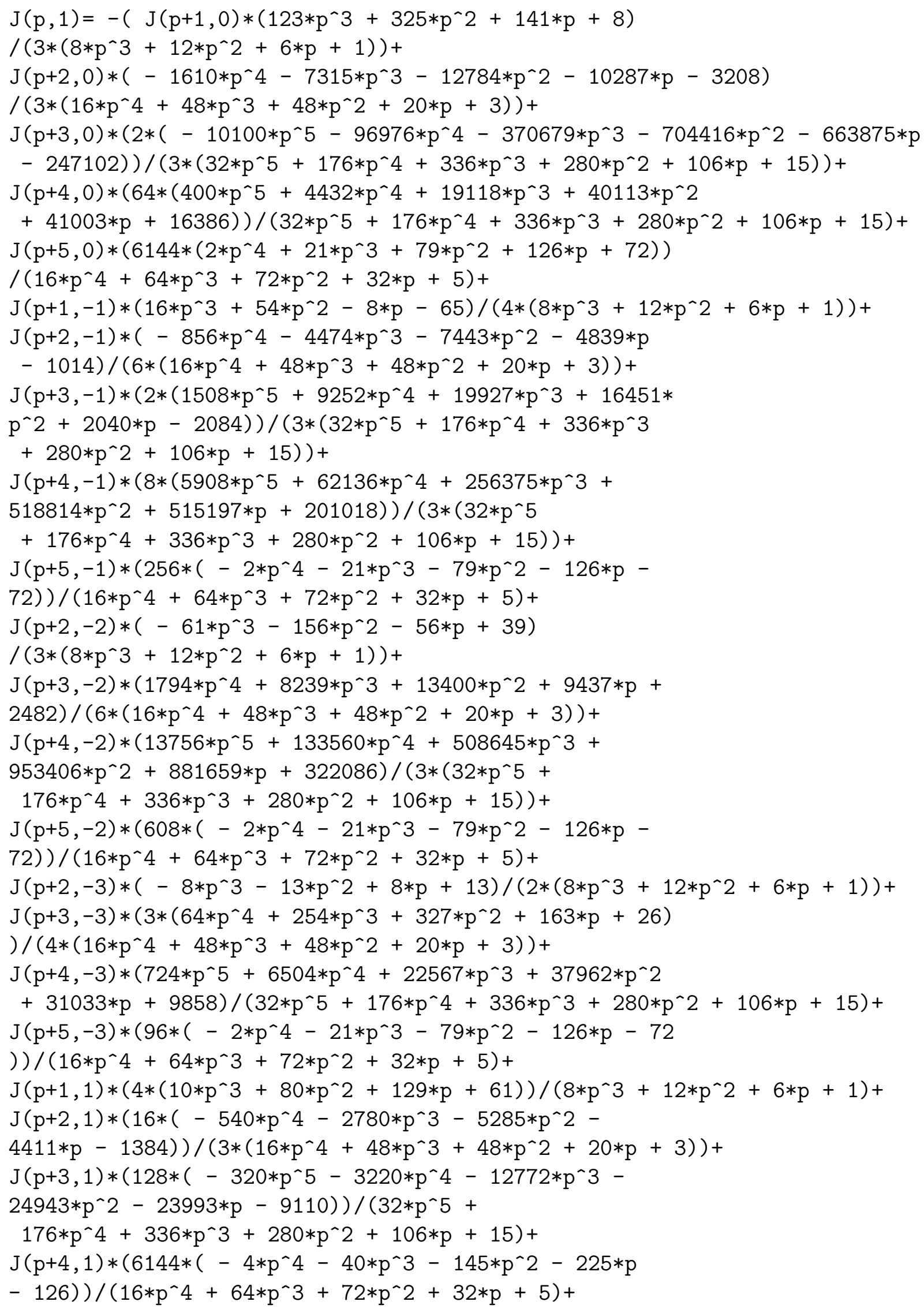




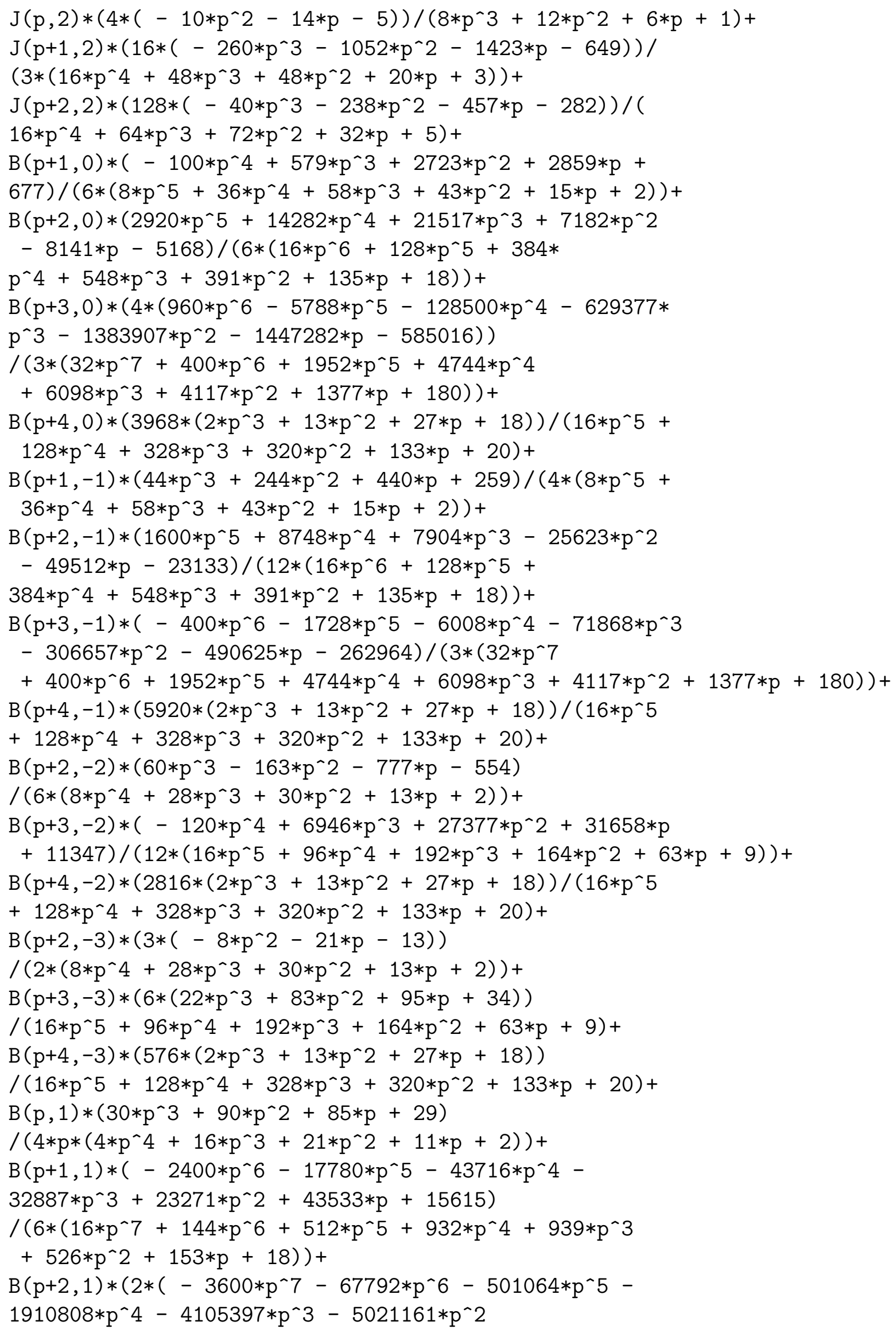




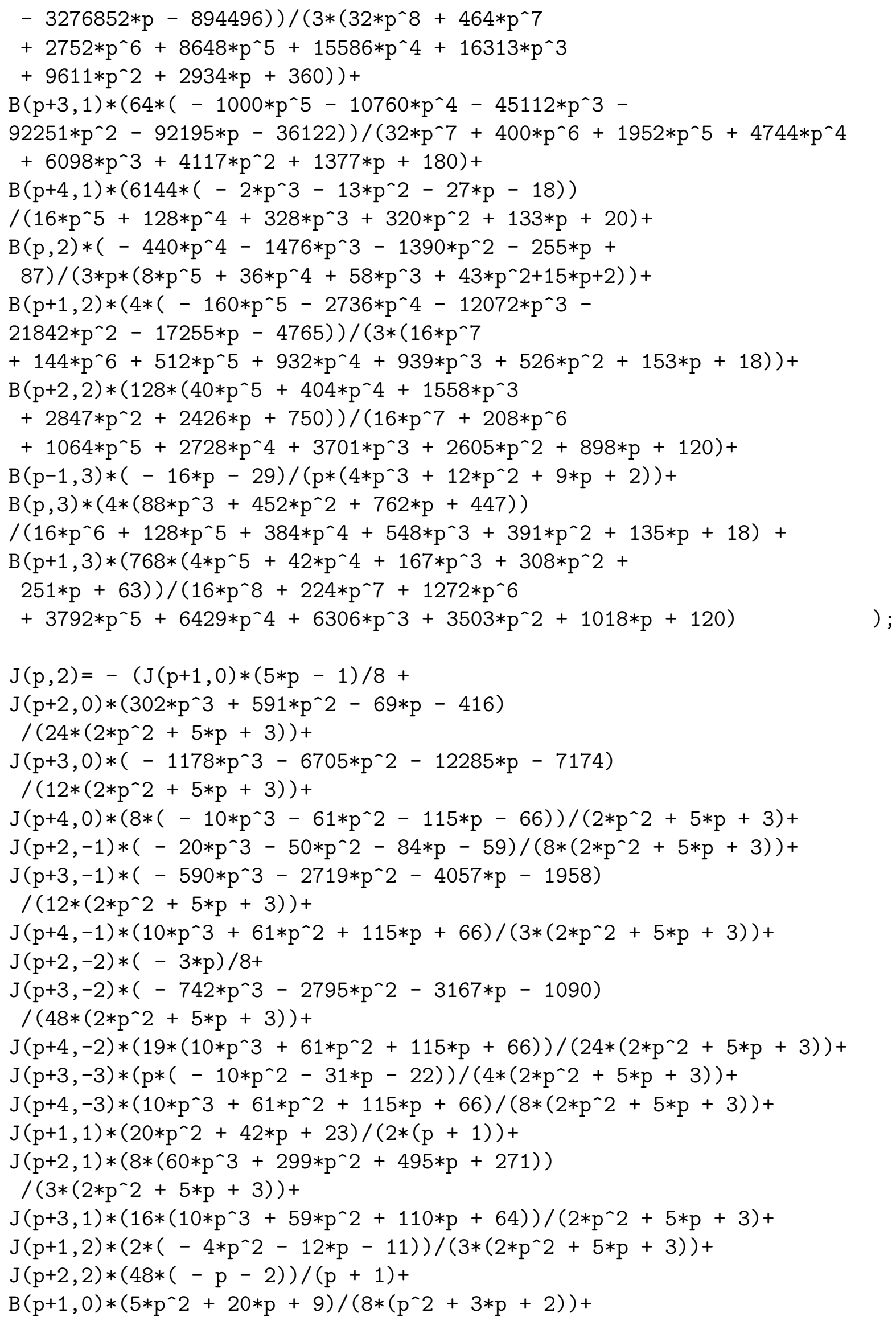




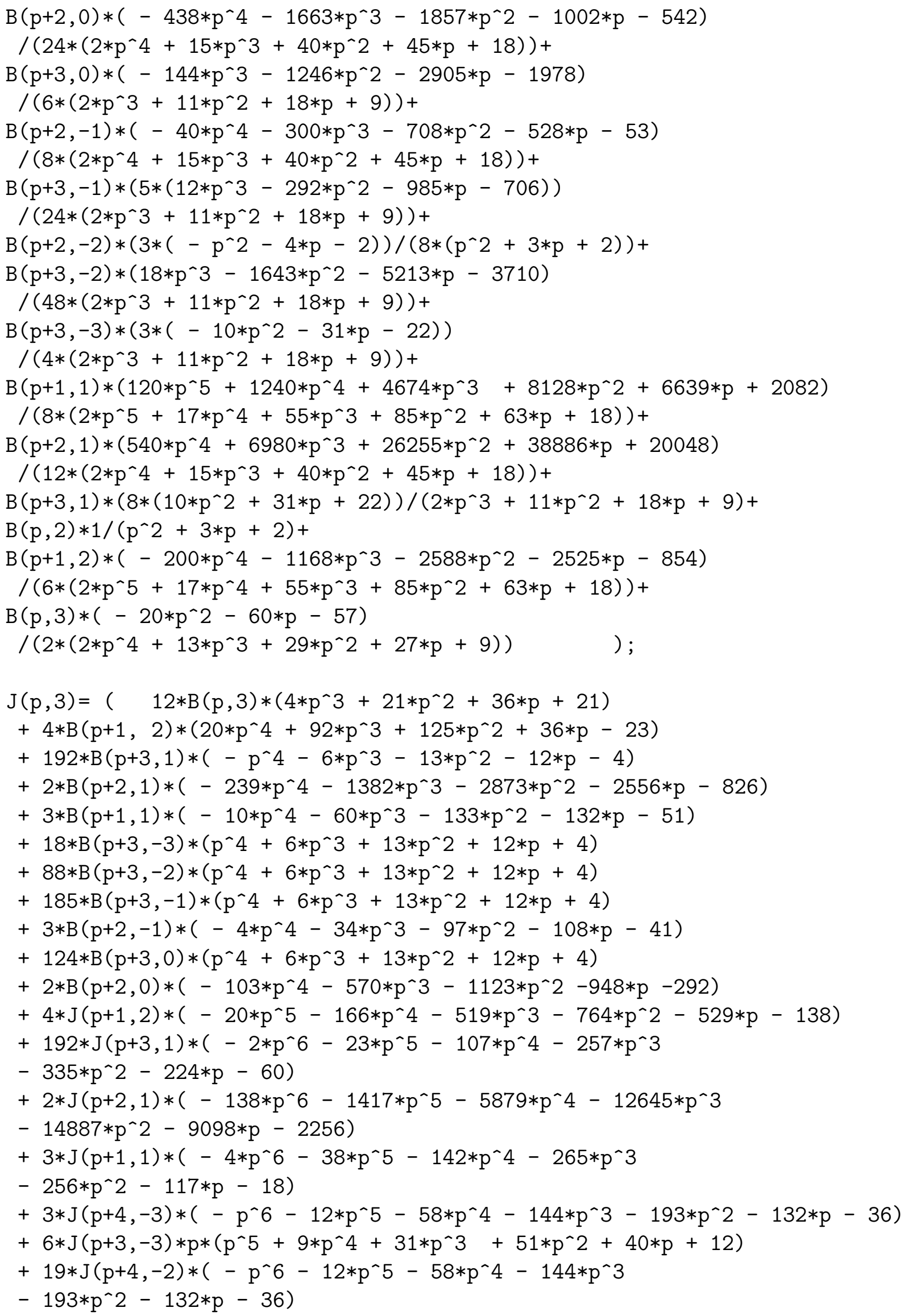




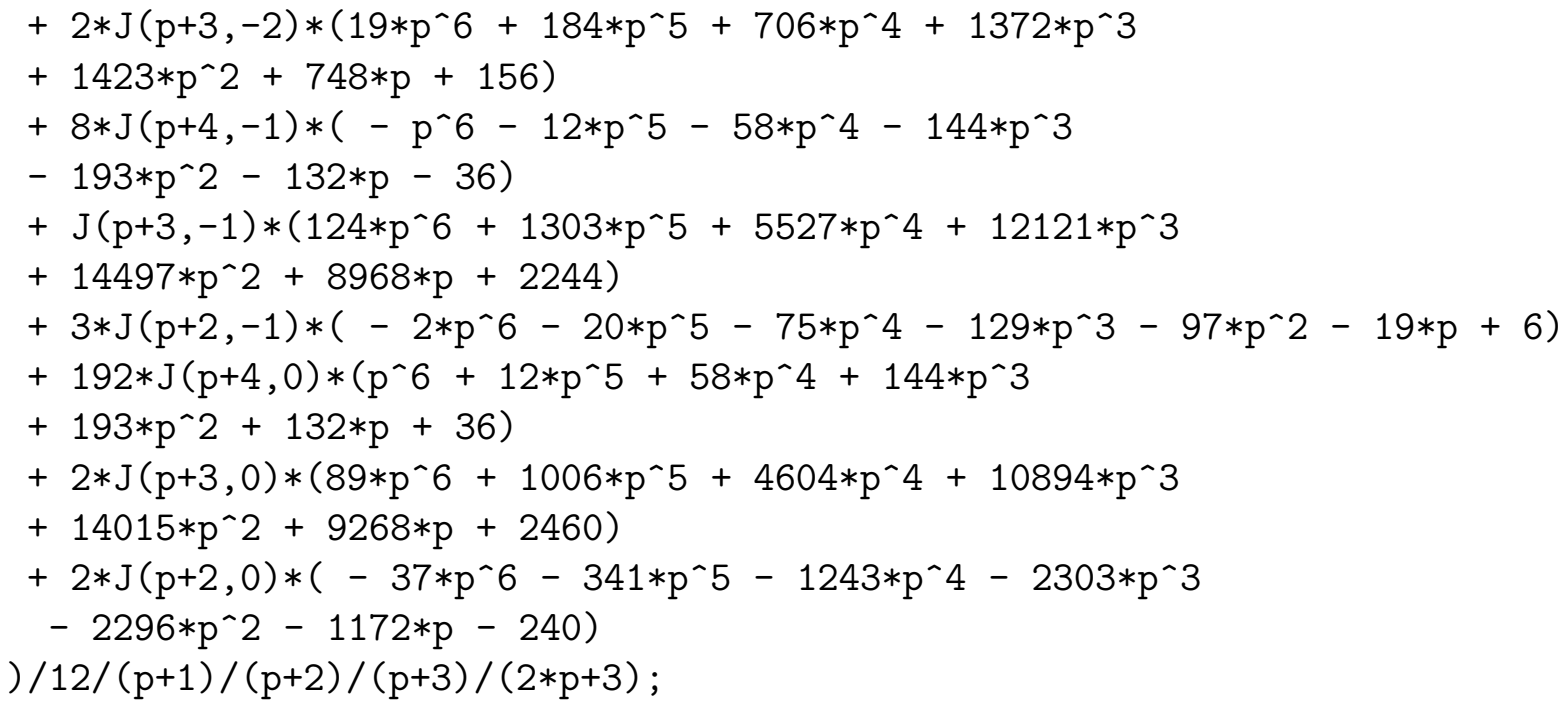

\section{Appendix 7.}

Here $B(0, q, n)$ is the coefficient of the expansion

$$
\mathcal{B}\left(0, q ; \mu_{B}^{2}\right)=\sum_{n=0}^{q-2} \frac{B(0, q, n)}{\left(\mu_{B}^{2}\right)^{n}}+D_{0}(0, q)\left(\ln \mu_{B}^{2}+C\right),
$$

(see (59) and ([60) ) and $D(p, q, r)$ is nothing but $D_{r}(p, q)$ defined in formula (59).

$$
\begin{aligned}
& \mathrm{Z}_{-} 0(\mathrm{q})=\left(\left(-414720 * \mathrm{q}^{\wedge} 2+5184000 * \mathrm{q}+105460920 /(\mathrm{q}-1)+22917120 /(\mathrm{q}-2)\right.\right. \\
& +1462140 /(\mathrm{q}-3)-337161300 /(\mathrm{q}+1)+5257440) * \mathrm{~B}(0, \mathrm{q}-4,0) \\
& +\left(2903040 * q^{2} 2-30274560 * q-350758440 /(q-1)-49733280 /(q-2)\right. \\
& -1462140 /(\mathrm{q}-3)+1236495780 /(\mathrm{q}+1)-26287200) * \mathrm{~B}(0, \mathrm{q}-3,1) \\
& +\left(8294400 * q^{\wedge} 2-84602880 * \mathrm{q}+426474720 /(\mathrm{q}-1)-12792960 /(\mathrm{q}-2)\right. \\
& -4267440 /(q-3)-1713368880 /(q+1)+420923520) * B(0, q-3,0) \\
& +\left(-8709120 * q^{2} 2+72783360 * q+388058400 /(q-1)+26816160 /(q-2)\right. \\
& -1679831040 /(\mathrm{q}+1)+52574400) * \mathrm{~B}(0, \mathrm{q}-2,2) \\
& +\left(-50595840 * q^{-2}+413061120 * \mathrm{q}-681805440 /(\mathrm{q}-1)+32651520 /(\mathrm{q}-2)\right. \\
& +4409120640 /(\mathrm{q}+1)-1598987520) * \mathrm{~B}(0, \mathrm{q}-2,1) \\
& +\left(-58060800 * q^{\wedge} 2+443750400 * q-1814227200 /(q-1)-29468160 /(q-2)\right. \\
& +8956742400 /(\mathrm{q}+1)-2136844800) * \mathrm{~B}(0, \mathrm{q}-2,0) \\
& +\left(14515200 * q^{-2}-91238400 * q-142760880 /(q-1)+1003590000 /(q+1)\right. \\
& -52574400) * \mathrm{~B}(0, \mathrm{q}-1,3) \\
& +\left(128563200 * q^{\wedge} 2-787968000 * q+234077760 /(q-1)-4178364480 /(q+1)\right. \\
& +2271421440) * \mathrm{~B}(0, \mathrm{q}-1,2) \\
& +\left(306892800 * q^{\wedge} 2-1758412800 * q+1767352320 /(q-1)-17987028480 /(q+1)\right. \\
& +6567989760) * \mathrm{~B}(0, \mathrm{q}-1,1) \\
& +\left(165888000 * q^{\wedge} 2-729907200 * q+388177920 /(q-1)-2986813440 /(q+1)\right. \\
& +1305262080) * \mathrm{~B}(0, \mathrm{q}-1,0) \\
& +\left(414720 * q^{\wedge} 2+829440 * q\right) * B(0, q+3,7) \\
& +\left(9123840 * \mathrm{q}^{\wedge} 2+18247680 * \mathrm{q}\right) * \mathrm{~B}(0, \mathrm{q}+3,6) \\
& +\left(74649600 * q^{\wedge} 2+149299200 * q\right) * B(0, q+3,5) \\
& +\left(282009600 * q^{\wedge} 2+564019200 * q\right) * B(0, q+3,4)
\end{aligned}
$$


$+\left(491028480 * \mathrm{q}^{\wedge} 2+982056960 * \mathrm{q}\right) * \mathrm{~B}(0, \mathrm{q}+3,3)$

$+\left(318504960 * \mathrm{q}^{\wedge} 2+637009920 * \mathrm{q}\right) * \mathrm{~B}(0, \mathrm{q}+3,2)$

$+\left(-2903040 * q^{\wedge} 2+207360 * q\right) * B(0, q+2,6)$

$+\left(-53913600 * \mathrm{q}^{\wedge} 2+1658880 * \mathrm{q}\right) * \mathrm{~B}(0, \mathrm{q}+2,5)$

$+\left(-356659200 * \mathrm{q}^{\wedge} 2-20736000 * \mathrm{q}\right) * \mathrm{~B}(0, \mathrm{q}+2,4)$

$+\left(-1011916800 * q^{\wedge} 2-265420800 * q\right) * B(0, q+2,3)$

$+\left(-1141309440 * q^{\wedge} 2-915701760 * q\right) * B(0, q+2,2)$

$+\left(-318504960 * \mathrm{q}^{2} 2-955514880 * \mathrm{q}\right) * \mathrm{~B}(0, \mathrm{q}+2,1)$

$+\left(8709120 * q^{\wedge} 2-18662400 * q+5257440 /(q+1)-5257440\right) * B(0, q+1,5)$

$+\left(132710400 * q^{\wedge} 2-273715200 * q-336216960 /(q+1)+336216960\right) * B(0, q+1,4)$

$+\left(680140800 * q^{\wedge} 2-1277337600 * q-2232368640 /(q+1)+2232368640\right) * B(0, q+1,3)$

$+\left(1343692800 * \mathrm{q}^{\wedge} 2-1891123200 * \mathrm{q}-2815395840 /(\mathrm{q}+1)\right.$

$+2815395840) * \mathrm{~B}(0, \mathrm{q}+1,2)$

$+\left(809533440 * q^{\wedge} 2+212336640 * q+3530096640 /(q+1)-3530096640\right) * B(0, q+1,1)$

$+(955514880 * \mathrm{q}+4140564480 /(\mathrm{q}+1)-4140564480) * \mathrm{~B}(0, \mathrm{q}+1,0)$

$+\left(-14515200 * \mathrm{q}^{\wedge} 2+61171200 * \mathrm{q}-228350880 /(\mathrm{q}+1)+26287200\right) * \mathrm{~B}(0, \mathrm{q}, 4)$

$+\left(-174182400 * q^{\wedge} 2+713318400 * \mathrm{q}+1824491520 /(\mathrm{q}+1)-1429574400\right) * \mathrm{~B}(0, \mathrm{q}, 3)$

$+\left(-646963200 * q^{\wedge} 2+2463436800 * q+11243888640 /(q+1)-6663513600\right) * B(0, q, 2)$

$+\left(-779673600 * q^{\wedge} 2+2322432000 * q+6705192960 /(q+1)-4209131520\right) * B(0, q, 1)$

$+\left(-159252480 * \mathrm{q}^{\wedge} 2-278691840 * \mathrm{q}-11515944960 /(\mathrm{q}+1)+5381406720\right) * \mathrm{~B}(0, \mathrm{q}, 0)$

$+(3034260 /(q-1)+706560 /(q-2)+32940 /(q-3)+604860 /(q-5)$

$-3963900 /(\mathrm{q}+1)) * \mathrm{D}(3, \mathrm{q}-8,1)$

$+(-22299660 /(q-1)-4327200 /(q-2)-164700 /(q-3)-2903328 /(q-4)$

$-1814580 /(q-5)+28191708 /(q+1)) * D(3, q-7,2)$

$+(2175210 /(q-1)+974320 /(q-2)+1169730 /(q-3)-1752336 /(q-4)$

$+3125110 /(\mathrm{q}-5)-12880514 /(\mathrm{q}+1)) * \mathrm{D}(3, \mathrm{q}-7,1)$

$+(70144380 /(q-1)+11037600 /(q-2)+5773140 /(q-3)+8709984 /(q-4)$

$+1814580 /(\mathrm{q}-5)-85867524 /(\mathrm{q}+1)) * \mathrm{D}(3, \mathrm{q}-6,3)$

$+(-36941910 /(q-1)-11226160 /(q-2)+1505790 /(q-3)-14098512 /(q-4)$

$-6149410 /(\mathrm{q}-5)+112114682 /(\mathrm{q}+1)) * \mathrm{D}(3, \mathrm{q}-6,2)$

$+(-59868660 /(q-1)+3139840 /(q-2)+3407220 /(q-3)-11231232 /(q-4)$

$+10685860 /(\mathrm{q}-5)+86076892 /(\mathrm{q}+1)) * \mathrm{D}(3, \mathrm{q}-6,1)$

$+(-122426100 /(q-1)-19848480 /(q-2)-16660620 /(q-3)-8709984 /(q-4)$

$-604860 /(\mathrm{q}-5)+145025724 /(\mathrm{q}+1)) * \mathrm{D}(3, \mathrm{q}-5,4)$

$+(98060730 /(q-1)+17506320 /(q-2)+24591150 /(q-3)+28615152 /(q-4)$

$+2721870 /(q-5)-303376182 /(q+1)) * D(3, q-5,3)$

$+(252260220 /(q-1)-25022880 /(q-2)+30365100 /(q-3)-48235296 /(q-4)$

- 604860/(q-5) - 349490604/(q+1))*D(3,q - 5,2)

$+(239320080 /(q-1)+52675200 /(q-2)+9501840 /(q-3)+87594624 /(q-4)$

$-16936080 /(\mathrm{q}-5)-306353424 /(\mathrm{q}+1)) * \mathrm{D}(3, \mathrm{q}-5,1)$

$+(129869280 /(q-1)+25993440 /(q-2)+16495920 /(q-3)+2903328 /(q-4)$

$-146231568 /(q+1)) * D(3, q-4,5)$

$+(-112768560 /(q-1)-32463600 /(q-2)-49388040 /(q-3)-12764304 /(q-4)$

$+425803704 /(\mathrm{q}+1)) * \mathrm{D}(3, \mathrm{q}-4,4)$

$+(-543929760 /(q-1)-33583200 /(q-2)+64962720 /(q-3)+1550304 /(q-4)$

$+828260736 /(q+1)) * D(3, q-4,3)$ 


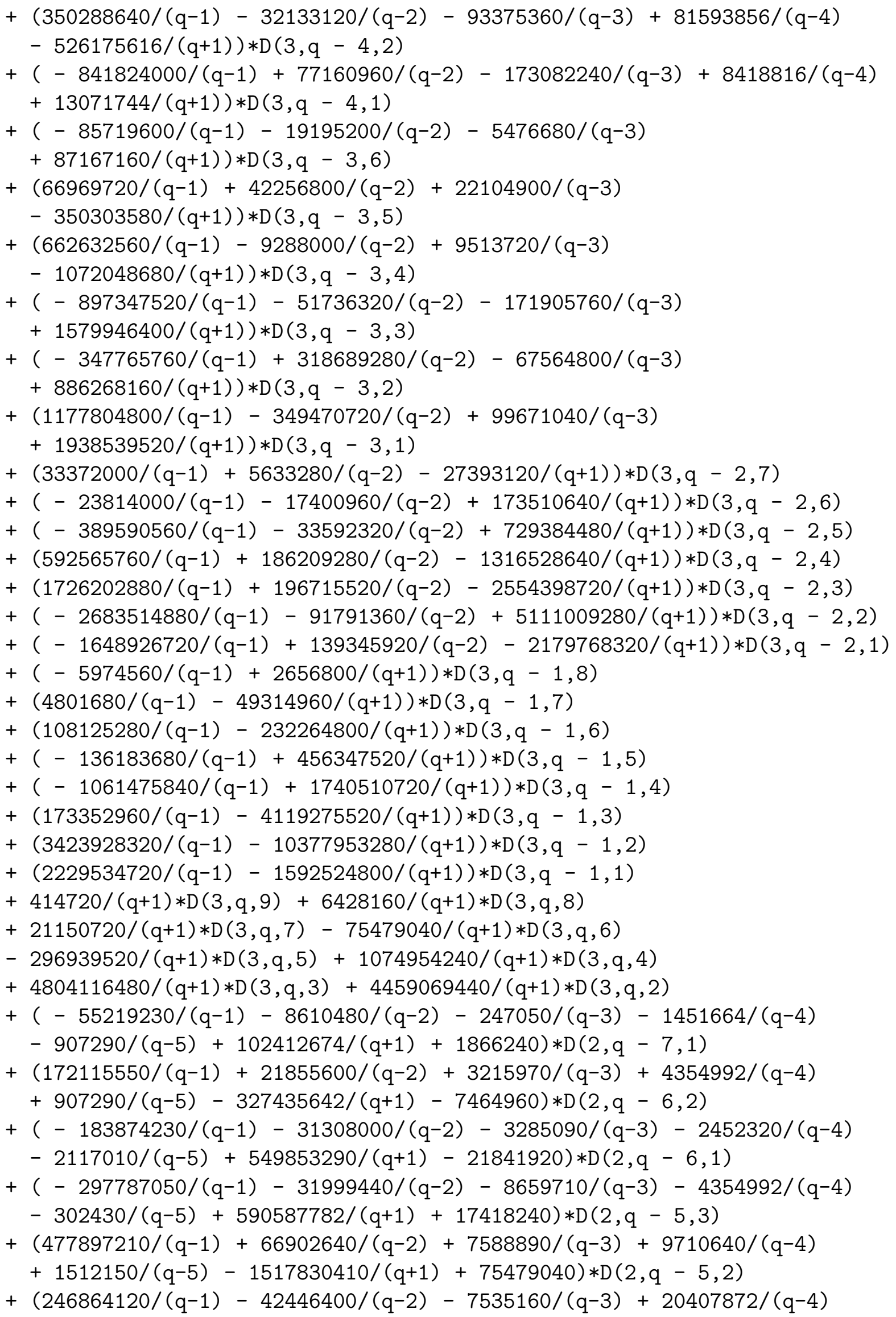




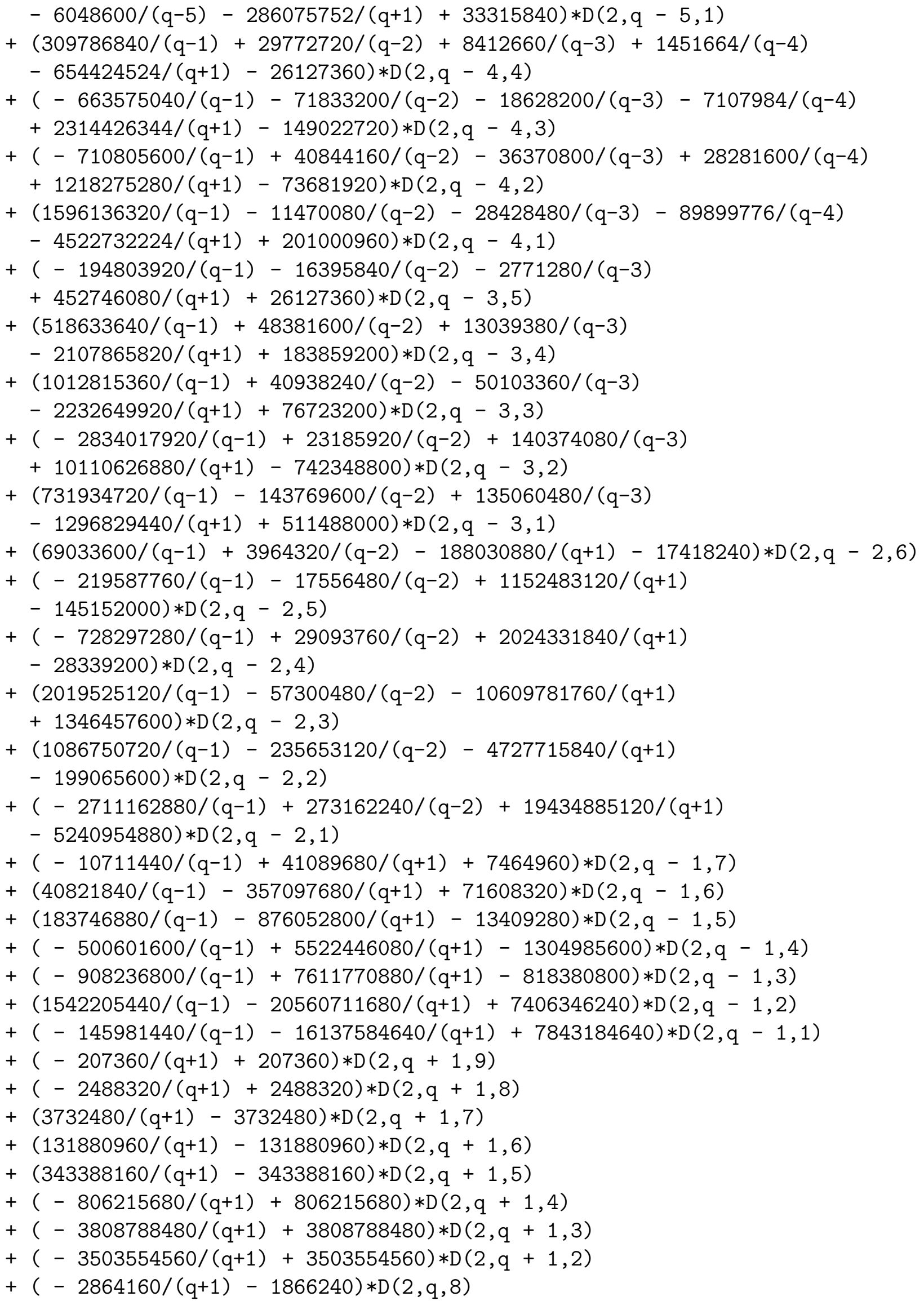




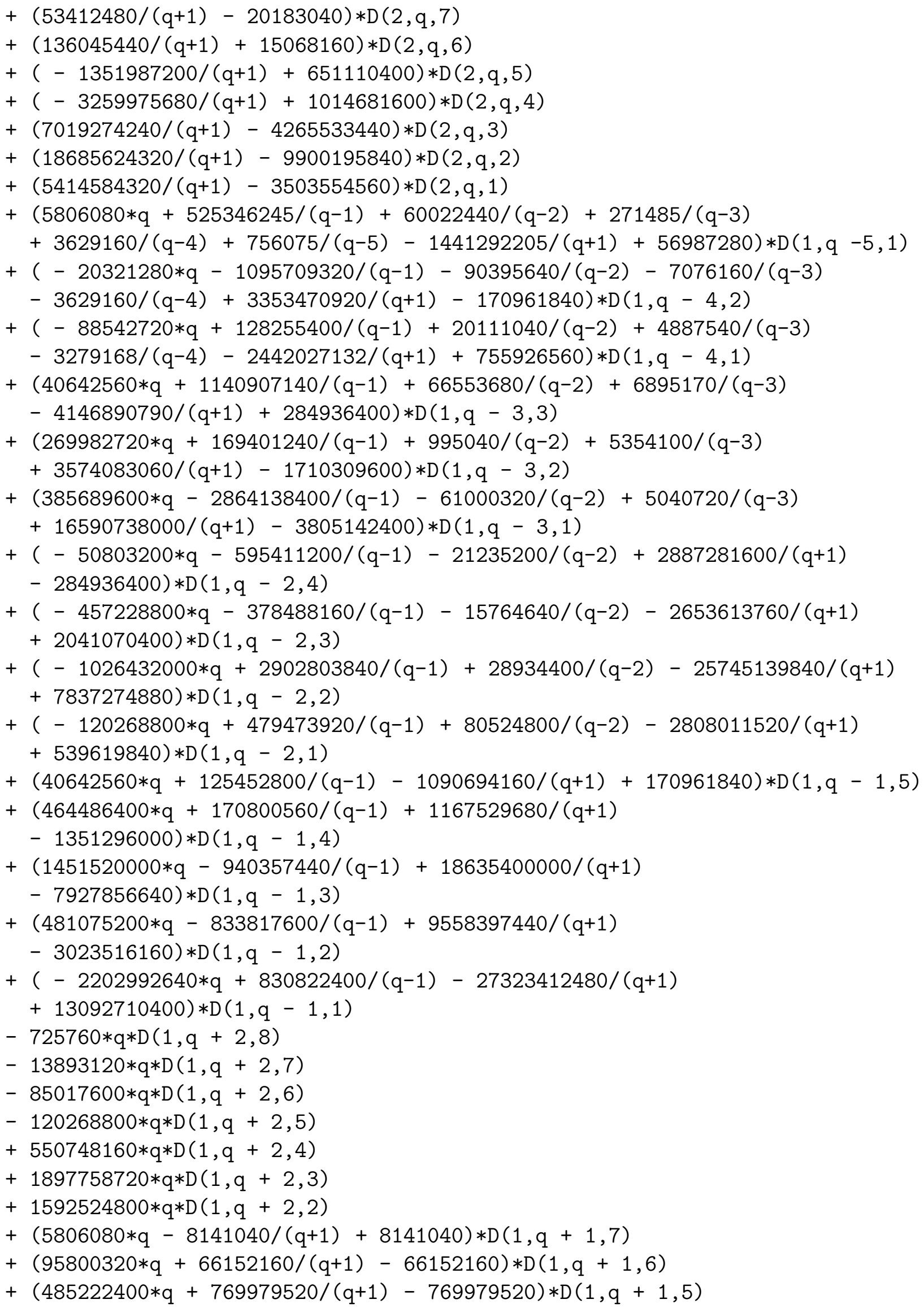


$+(481075200 * \mathrm{q}+1112209920 /(\mathrm{q}+1)-1112209920) * \mathrm{D}(1, \mathrm{q}+1,4)$

$+(-2202992640 * q-3838924800 /(q+1)+3838924800) * D(1, q+1,3)$

$+(-4591779840 * \mathrm{q}-9196830720 /(\mathrm{q}+1)+9196830720) * \mathrm{D}(1, \mathrm{q}+1,2)$

$+(-1592524800 * q-3822059520 /(q+1)+3822059520) * D(1, q+1,1)$

$+(-20321280 * \mathrm{q}+189367200 /(\mathrm{q}+1)-56987280) * \mathrm{D}(1, \mathrm{q}, 6)$

$+(-283046400 * \mathrm{q}-362849760 /(\mathrm{q}+1)+468715680) * \mathrm{D}(1, \mathrm{q}, 5)$

$+(-1150848000 * \mathrm{q}-6244715520 /(\mathrm{q}+1)+3941015040) * \mathrm{D}(1, \mathrm{q}, 4)$

$+(-721612800 * q-6432307200 /(q+1)+3339394560) * D(1, q, 3)$

$+(3304488960 * \mathrm{q}+18636963840 /(\mathrm{q}+1)-12536709120) * \mathrm{D}(1, \mathrm{q}, 2)$

$+(3490283520 * \mathrm{q}+19292774400 /(\mathrm{q}+1)-13052067840) * \mathrm{D}(1, \mathrm{q}, 1)$

$+\left(2903040 * q^{2} 2-27786240 * q-41109120 /(q-1)-3663360 /(q-2)+285120 /(q-3)\right.$

$-8389440 /(q+1)+81907200) * D(0, q-3,1)$

$+\left(-8709120 * q^{-2}+66562560 * q+43856640 /(q-1)+1451520 /(q-2)\right.$

$+74960640 /(\mathrm{q}+1)-163814400) * \mathrm{D}(0, \mathrm{q}-2,2)$

$+\left(-50595840 * q^{2} 2+396472320 * \mathrm{q}+249246720 /(\mathrm{q}-1)+14929920 /(\mathrm{q}-2)\right.$

$+498908160 /(q+1)-999475200) * D(0, q-2,1)$

$+\left(14515200 * q^{\wedge} 2-82944000 * q-15344640 /(q-1)-119439360 /(q+1)\right.$

$+163814400) * D(0, q-1,3)$

$+\left(128563200 * q^{\wedge} 2-754790400 * q-135613440 /(q-1)-1170754560 /(q+1)\right.$

$+1530316800) * D(0, q-1,2)$

$+\left(306892800 * q^{\wedge} 2-1924300800 * q-282839040 /(q-1)-3881779200 /(q+1)\right.$

$+4227655680) * \mathrm{D}(0, \mathrm{q}-1,1)$

$+\left(414720 * \mathrm{q}^{\wedge} 2+829440 * \mathrm{q}\right) * \mathrm{D}(0, \mathrm{q}+3,7)$

$+\left(9123840 * \mathrm{q}^{\wedge} 2+18247680 * \mathrm{q}\right) * \mathrm{D}(0, \mathrm{q}+3,6)$

$+\left(74649600 * q^{\wedge} 2+149299200 * q\right) * D(0, q+3,5)$

$+\left(282009600 * \mathrm{q}^{\wedge} 2+564019200 * \mathrm{q}\right) * \mathrm{D}(0, \mathrm{q}+3,4)$

$+\left(491028480 * \mathrm{q}^{\wedge} 2+982056960 * \mathrm{q}\right) * \mathrm{D}(0, \mathrm{q}+3,3)$

$+\left(318504960 * \mathrm{q}^{\wedge} 2+637009920 * \mathrm{q}\right) * \mathrm{D}(0, \mathrm{q}+3,2)$

$+\left(-2903040 * q^{\wedge} 2-207360 * q\right) * D(0, q+2,6)$

$+\left(-53913600 * \mathrm{q}^{\wedge} 2-1658880 * \mathrm{q}\right) * \mathrm{D}(0, \mathrm{q}+2,5)$

$+\left(-356659200 * q^{\wedge} 2+20736000 * q\right) * D(0, q+2,4)$

$+\left(-1011916800 * q^{\wedge} 2+265420800 * q\right) * D(0, q+2,3)$

$+\left(-1141309440 * q^{\wedge} 2+915701760 * q\right) * D(0, q+2,2)$

$+\left(-318504960 * \mathrm{q}^{-2}+955514880 * \mathrm{q}\right) * \mathrm{D}(0, \mathrm{q}+2,1)$

$+\left(8709120 * q^{2} 2-16174080 * q-16381440 /(q+1)+16381440\right) * D(0, q+1,5)$

$+\left(132710400 * q^{\wedge} 2-257126400 * q-265420800 /(q+1)+265420800\right) * D(0, q+1,4)$

$+\left(680140800 * q^{2} 2-1443225600 * q-1575106560 /(q+1)+1575106560\right) * D(0, q+1,3)$

$+\left(1343692800 * q^{\wedge} 2-3483648000 * \mathrm{q}-4283228160 /(\mathrm{q}+1)\right.$

$+4283228160) * \mathrm{D}(0, \mathrm{q}+1,2)$

$+\left(809533440 * q^{\wedge} 2-3450470400 * q-5202247680 /(q+1)+5202247680\right) * D(0, q+1,1)$

$+\left(-14515200 * q^{\wedge} 2+54950400 * q+74649600 /(q+1)-81907200\right) * D(0, q, 4)$

$+\left(-174182400 * \mathrm{q}^{\wedge} 2+680140800 * \mathrm{q}+970444800 /(\mathrm{q}+1)-1040947200\right) * \mathrm{D}(0, \mathrm{q}, 3)$

$+\left(-646963200 * q^{\wedge} 2+2712268800 * q+4494735360 /(q+1)-4476487680\right) * D(0, q, 2)$

$+\left(-779673600 * \mathrm{q}^{\wedge} 2+3914956800 * \mathrm{q}+8944680960 /(\mathrm{q}+1)-7697203200\right) * \mathrm{D}(0, \mathrm{q}, 1)$

$+(1517130 /(q-1)+353280 /(q-2)+16470 /(q-3)+302430 /(q-5)$

$-1981950 /(q+1)) * J(3, q-8)$ 


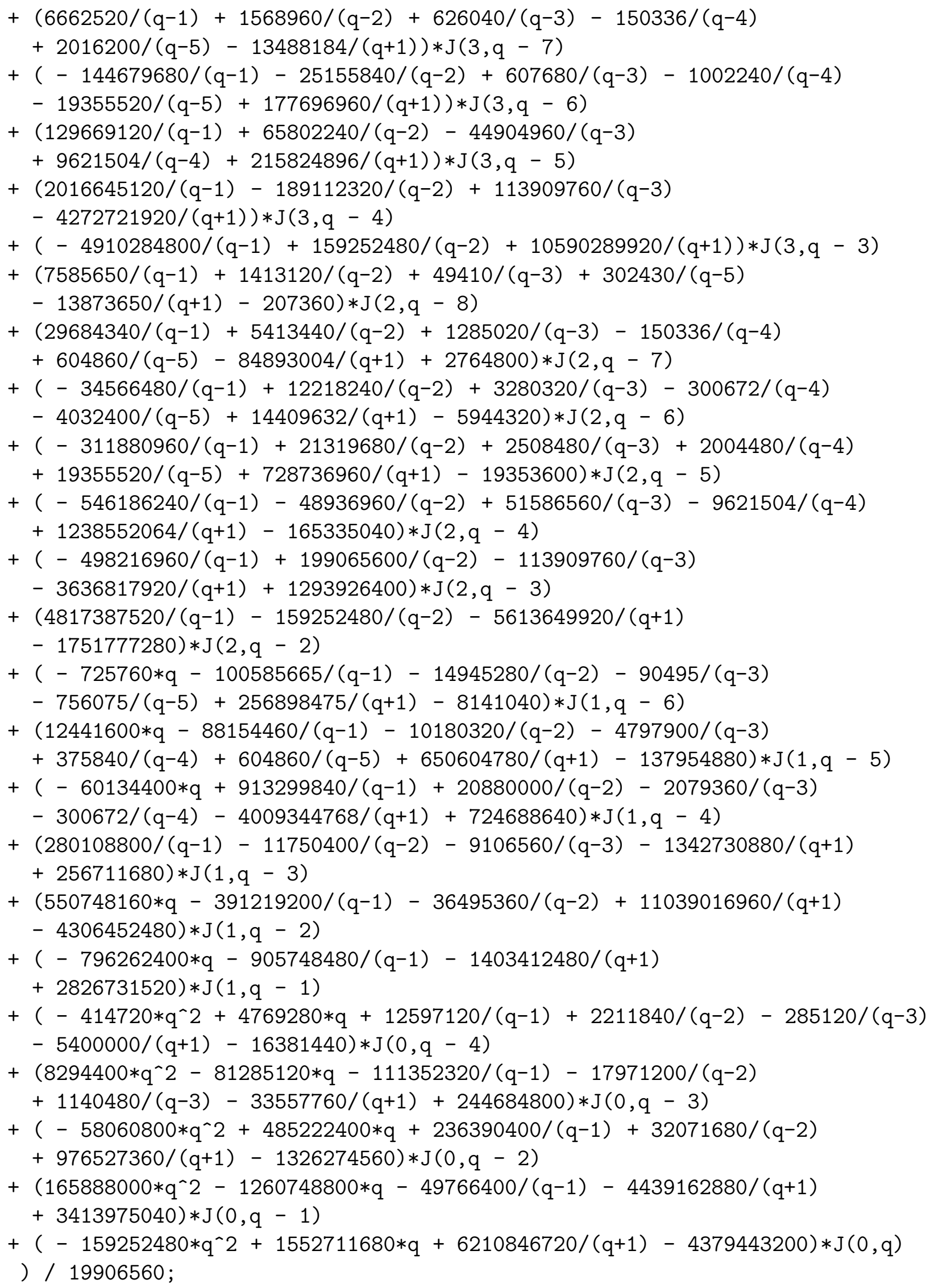




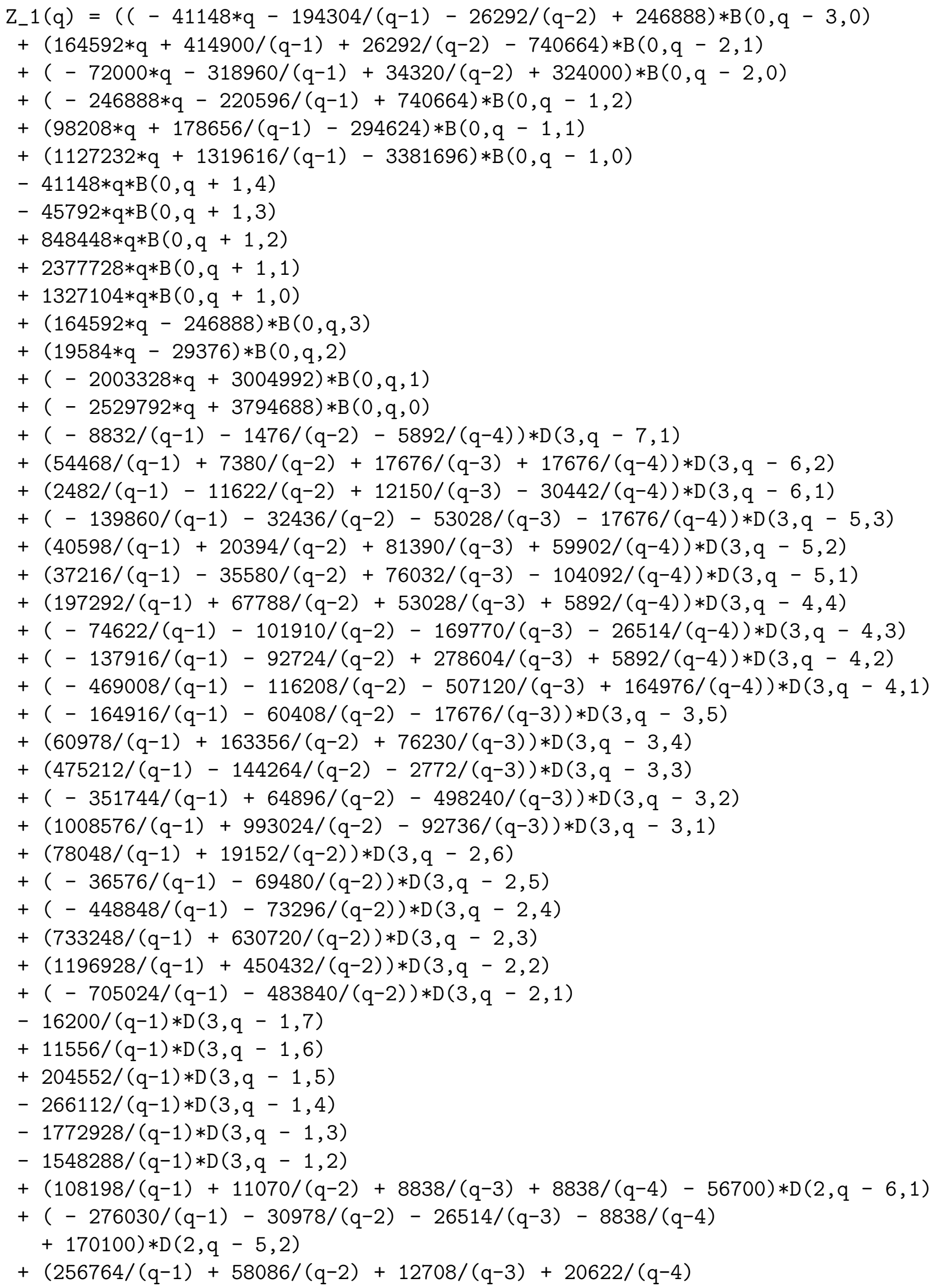




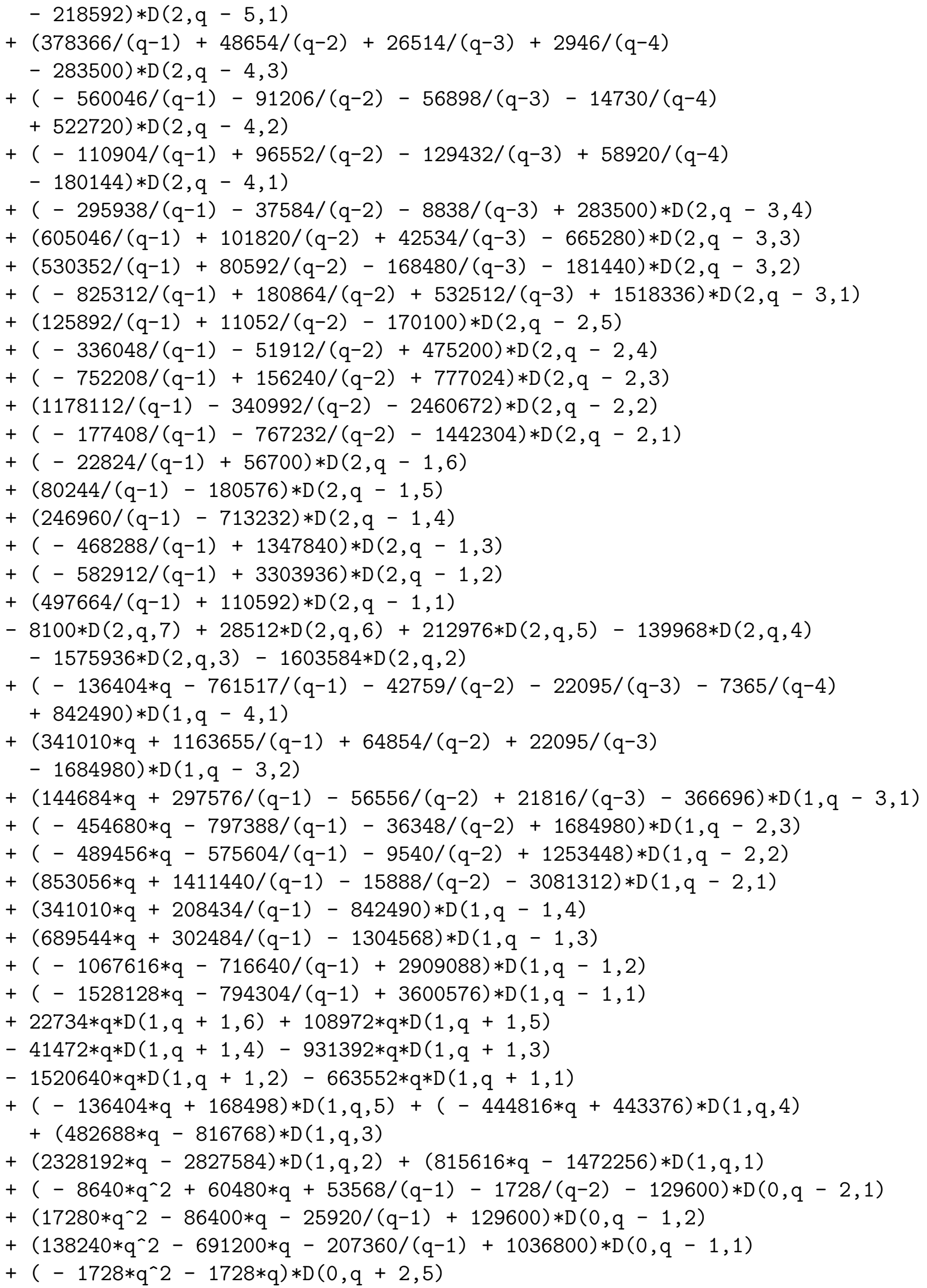




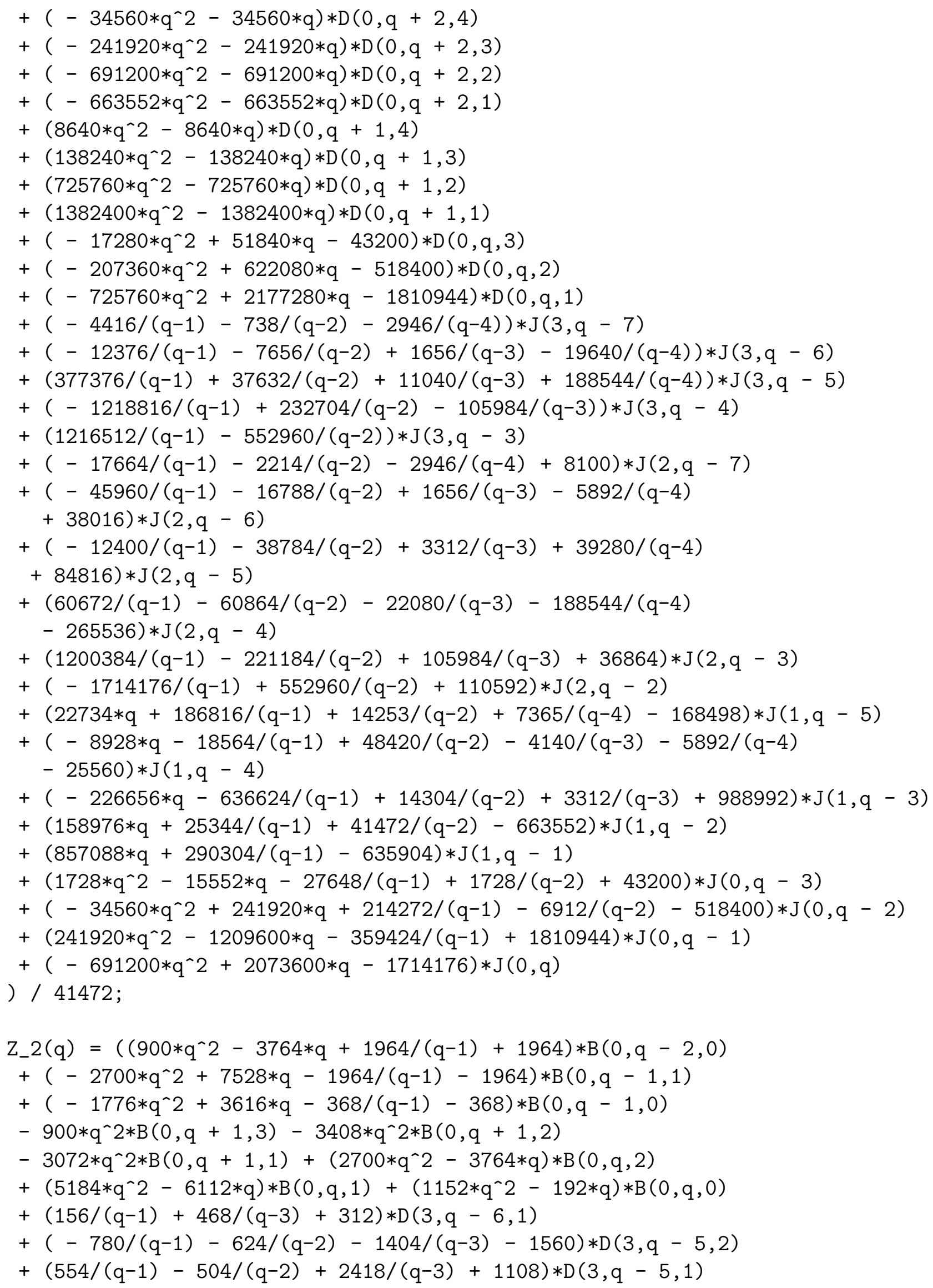




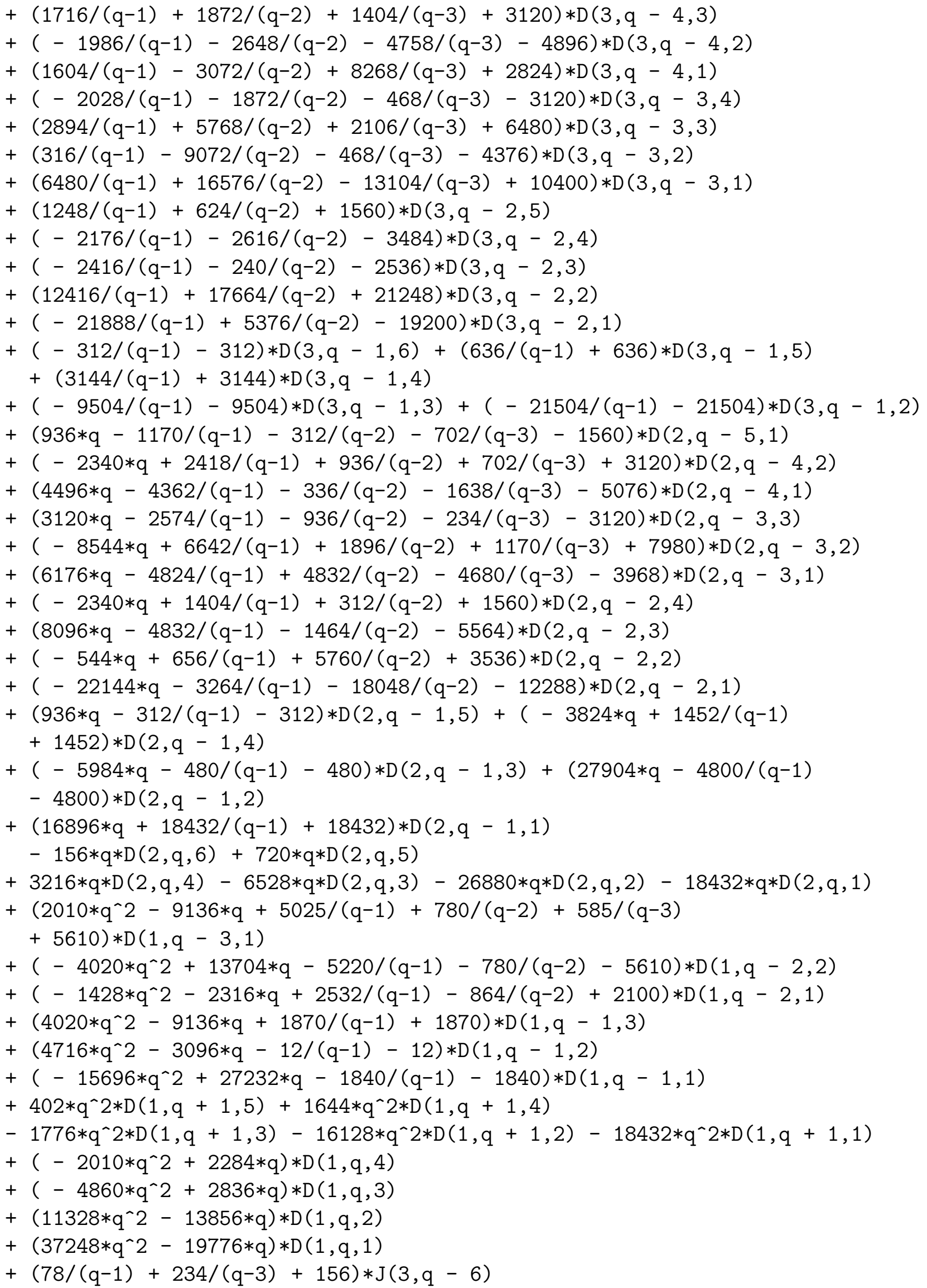




$$
\begin{aligned}
& +(472 /(\mathrm{q}-1)-96 /(\mathrm{q}-2)+1560 /(\mathrm{q}-3)+944) * \mathrm{~J}(3, \mathrm{q}-5) \\
& +(-5312 /(\mathrm{q}-1)-640 /(\mathrm{q}-2)-14976 /(\mathrm{q}-3)-10624) * \mathrm{~J}(3, \mathrm{q}-4) \\
& +(3072 /(\mathrm{q}-1)+6144 /(\mathrm{q}-2)+6144) * \mathrm{~J}(3, \mathrm{q}-3) \\
& +(-156 * \mathrm{q}+234 /(\mathrm{q}-1)+234 /(\mathrm{q}-3)+312) * \mathrm{~J}(2, \mathrm{q}-6) \\
& +(-944 * \mathrm{q}+1100 /(\mathrm{q}-1)-96 /(\mathrm{q}-2)+468 /(\mathrm{q}-3)+1208) * \mathrm{~J}(2, \mathrm{q}-5) \\
& +(-2864 * \mathrm{q}+2048 /(\mathrm{q}-1)-192 /(\mathrm{q}-2)-3120 /(\mathrm{q}-3)+912) * \mathrm{~J}(2, \mathrm{q}-4) \\
& +(1536 * \mathrm{q}+5056 /(\mathrm{q}-1)+1280 /(\mathrm{q}-2)+14976 /(\mathrm{q}-3)+10688) * \mathrm{~J}(2, \mathrm{q}-3) \\
& +(23808 * \mathrm{q}-7680 /(\mathrm{q}-1)-6144 /(\mathrm{q}-2)-10752) * \mathrm{~J}(2, \mathrm{q}-2) \\
& +(-402 * \mathrm{q}-2+2284 * \mathrm{q}-1675 /(\mathrm{q}-1)-585 /(\mathrm{q}-3)-1870) * \mathrm{~J}(1, \mathrm{q}-4) \\
& +(-72 * \mathrm{q}-2+2576 * \mathrm{q}-2364 /(\mathrm{q}-1)+240 /(\mathrm{q}-2)+468 /(\mathrm{q}-3)-2088) * \mathrm{~J}(1, \mathrm{q}-3) \\
& +(6144 * \mathrm{q}-2-11648 * \mathrm{q}+448 /(\mathrm{q}-1)-192 /(\mathrm{q}-2)+352) * \mathrm{~J}(1, \mathrm{q}-2) \\
& +(-19200 * \mathrm{q}-2+9984 * \mathrm{q}+1152 /(\mathrm{q}-1)+1152) * \mathrm{~J}(1, \mathrm{q}-1) \\
& +/ 1152 ;
\end{aligned}
$$

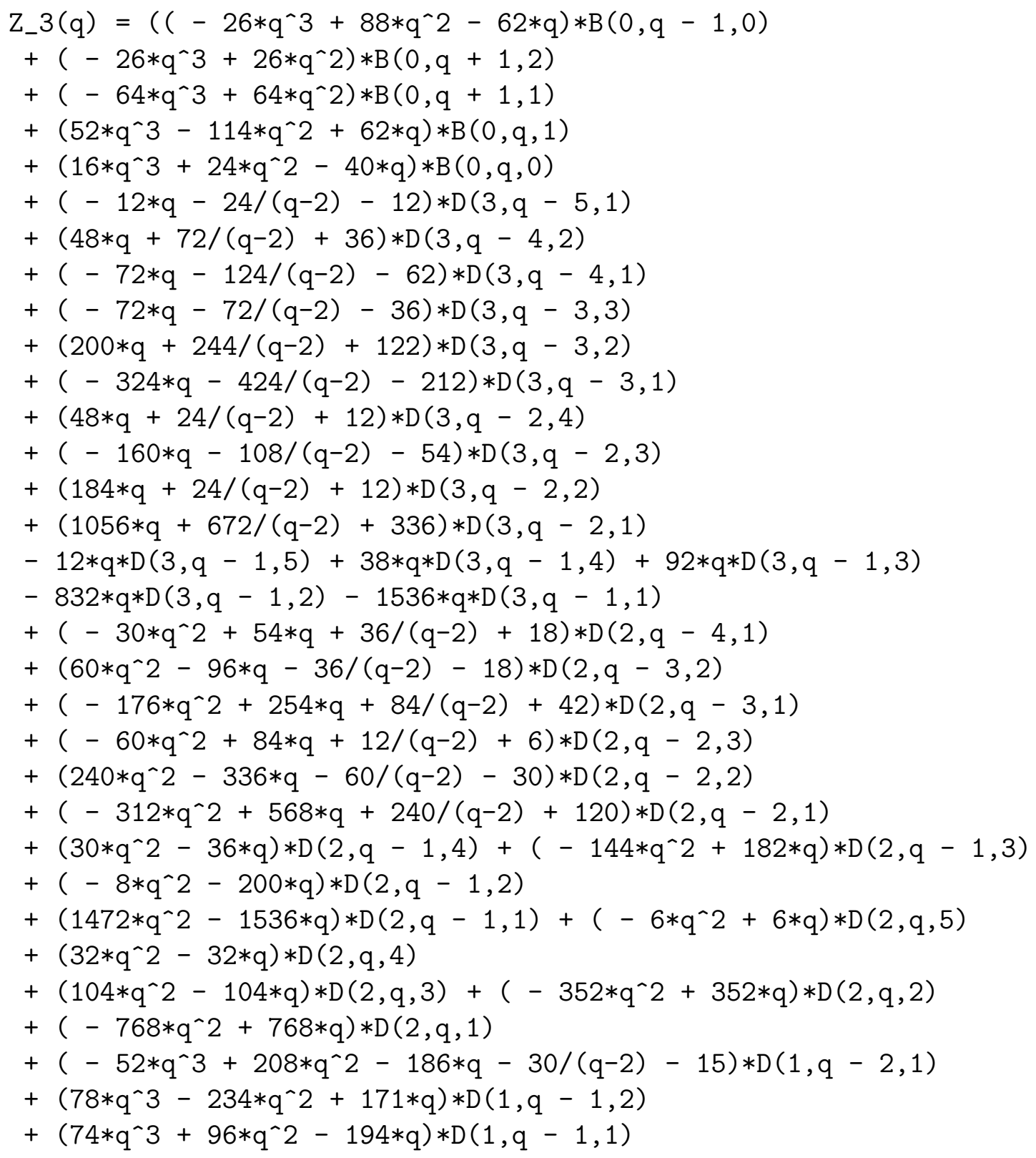




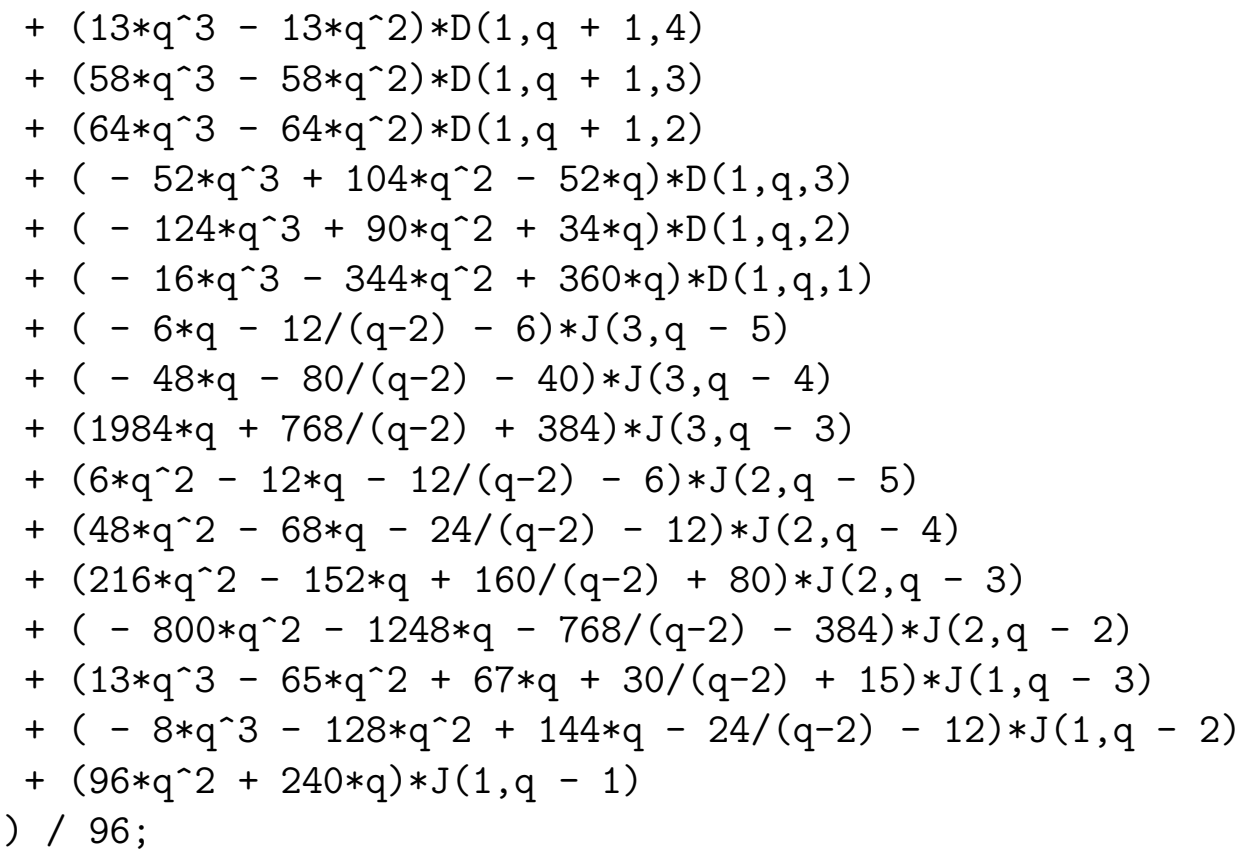

\section{Appendix 8 .}

Here and below, the symbol $\mathrm{JJ}(\mathrm{p}, \mathrm{q})$ in any Appendix designates $\mathcal{J}(p, q)$, whereas $\mathrm{J}(\mathrm{p}, \mathrm{q})$ designates $J(p, q)$. In computations at $q \leq 0, \mu_{B}=\mathrm{muB}=0$ and $\mathrm{JJ}(\mathrm{p}, \mathrm{q})$ should be replaced with $\mathrm{J}(\mathrm{p}, \mathrm{q})$.

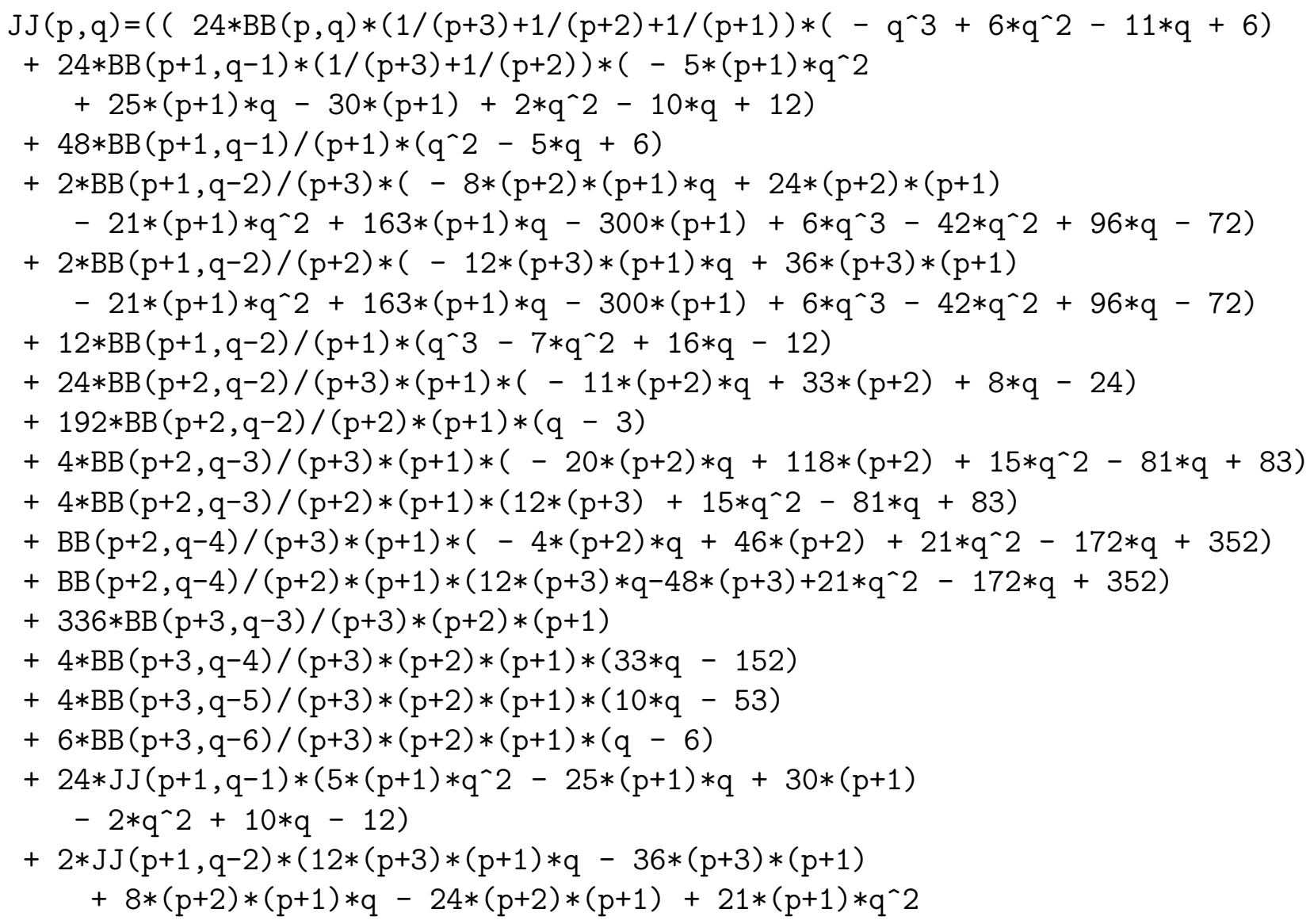




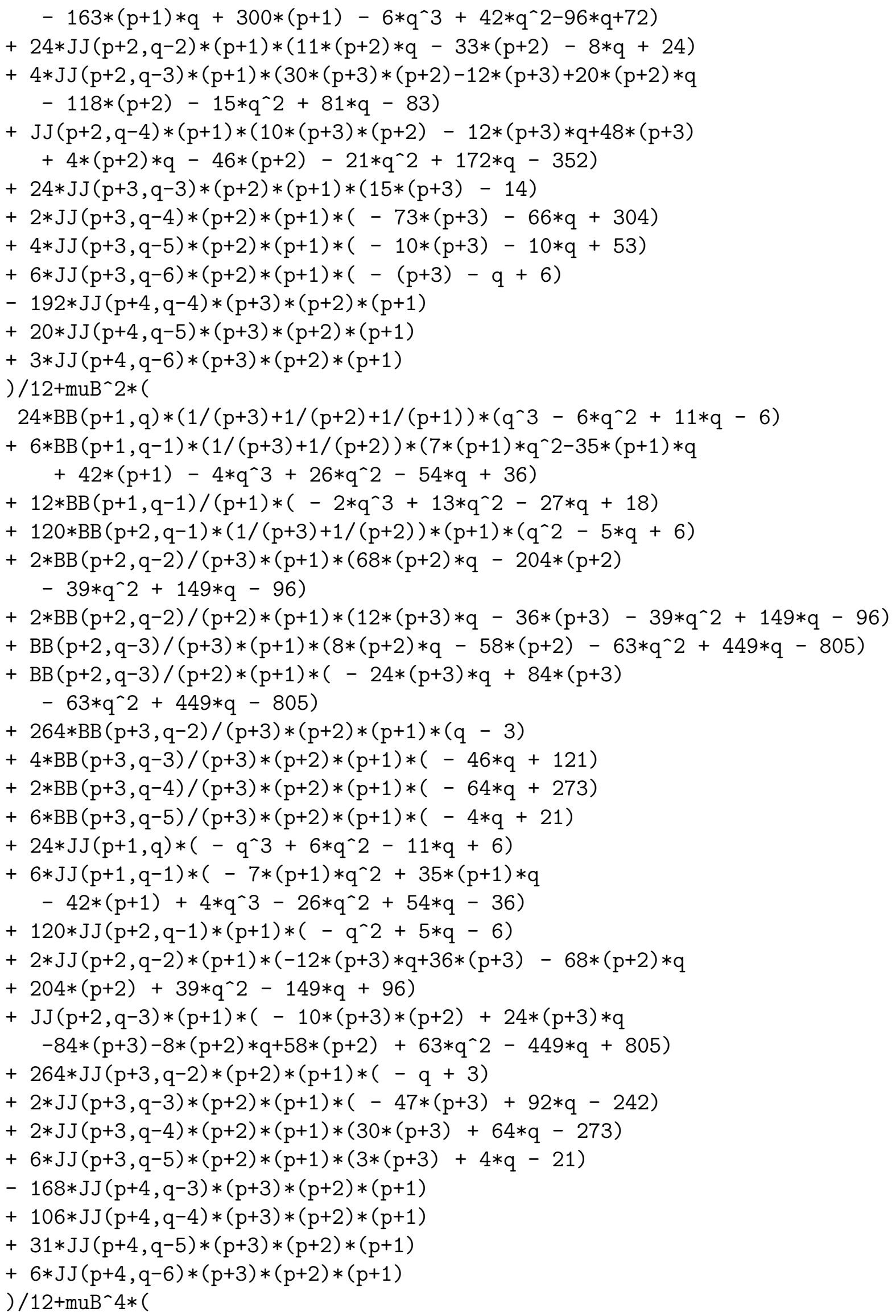




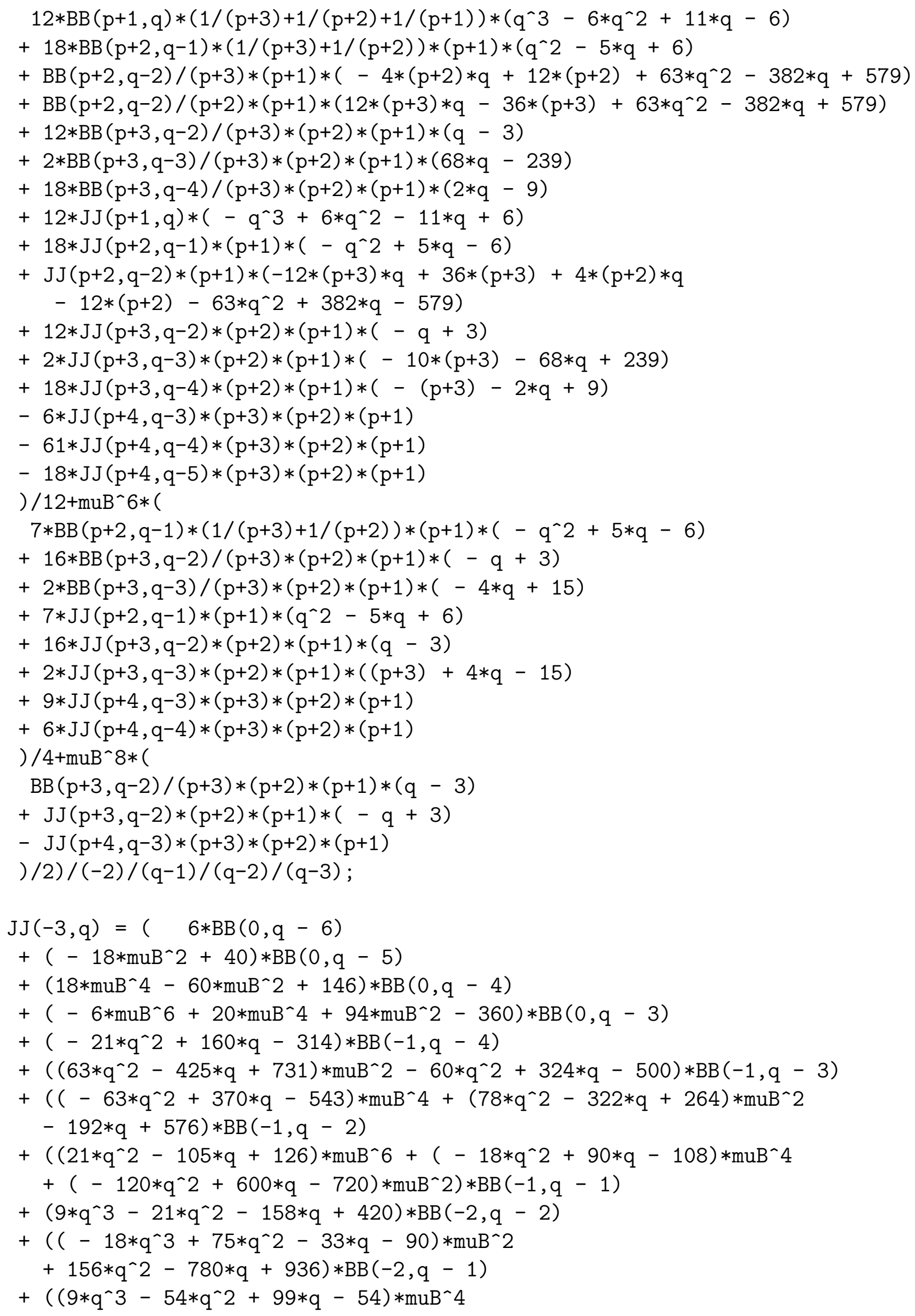




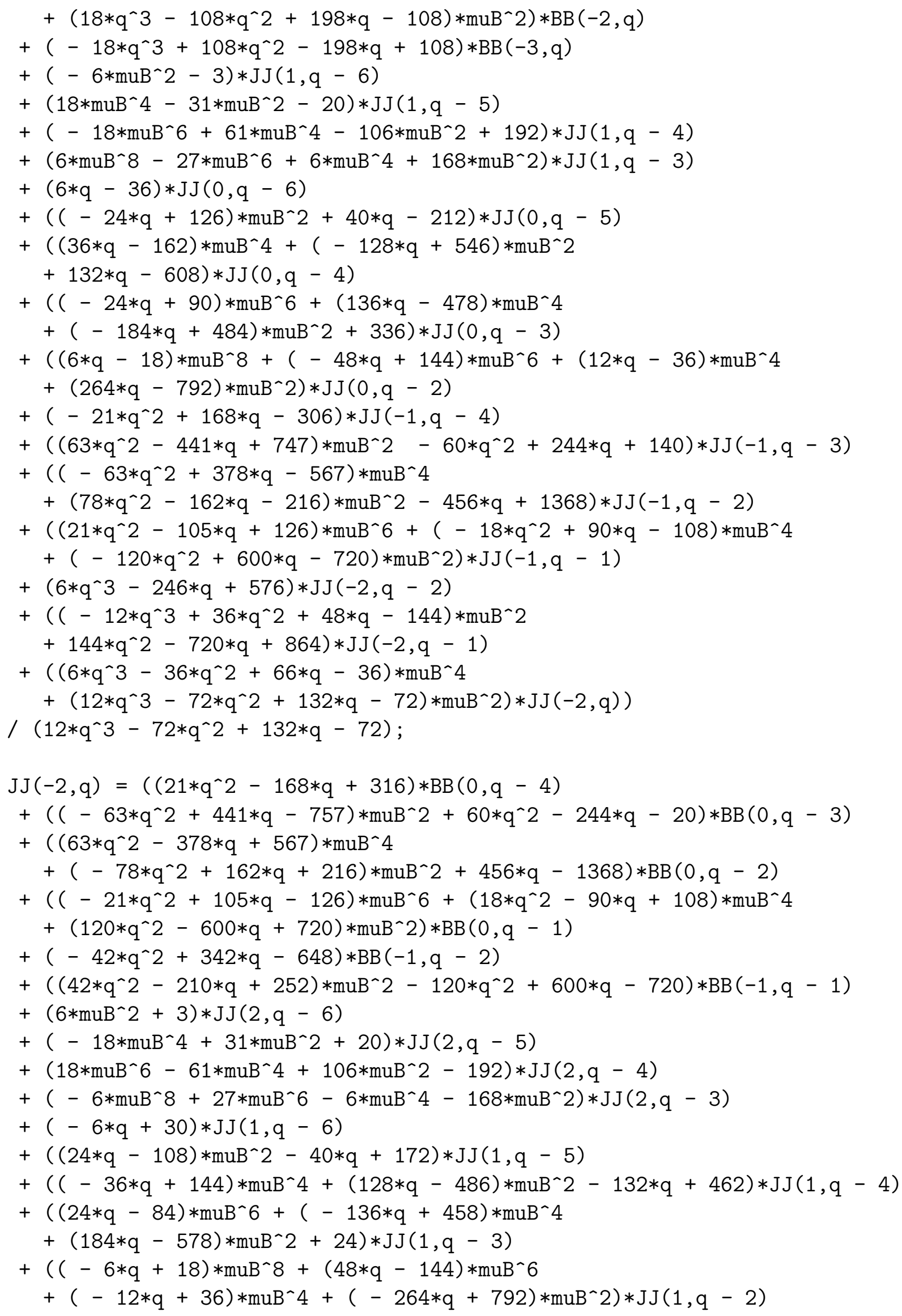




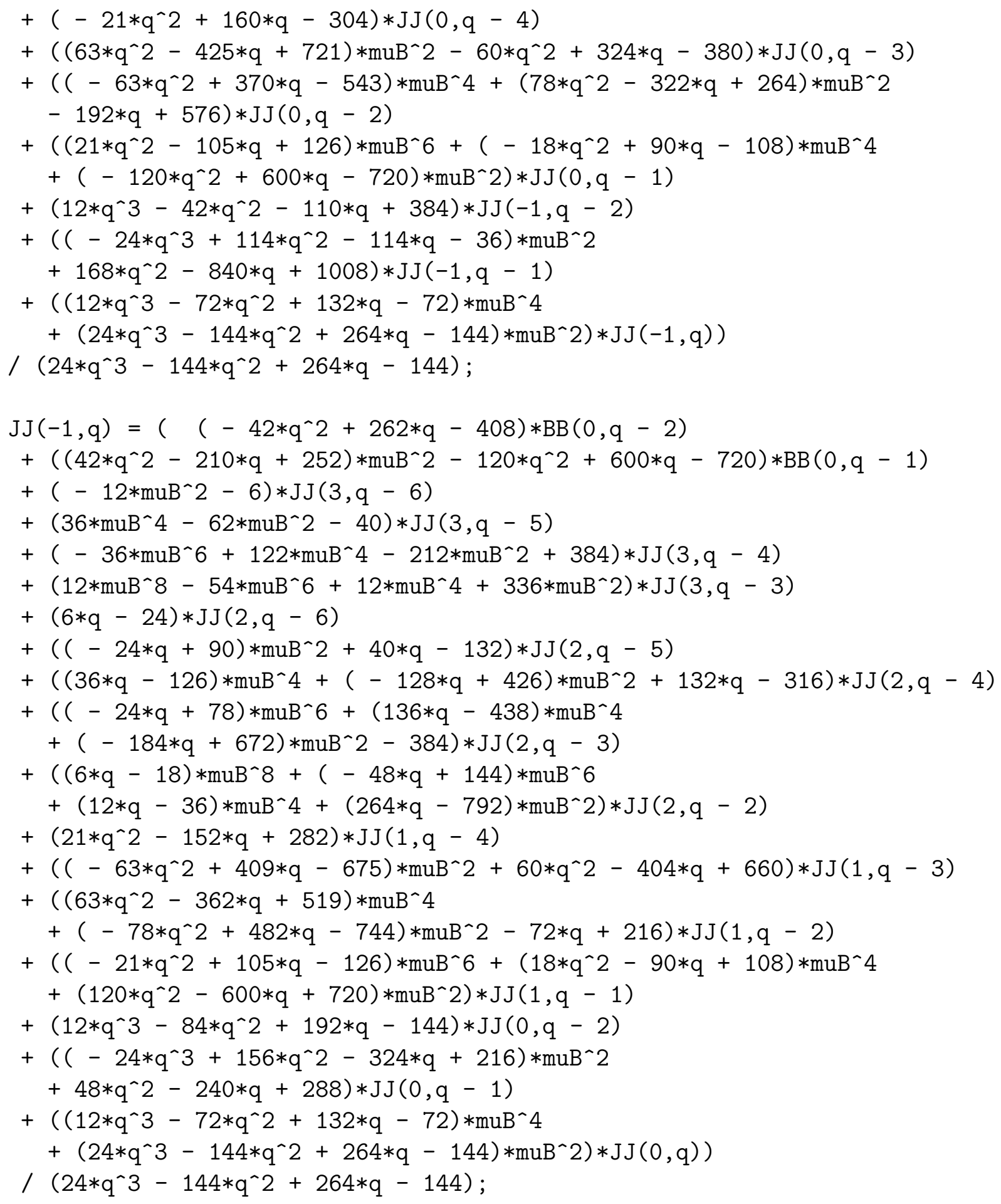

\section{Appendix 9.}

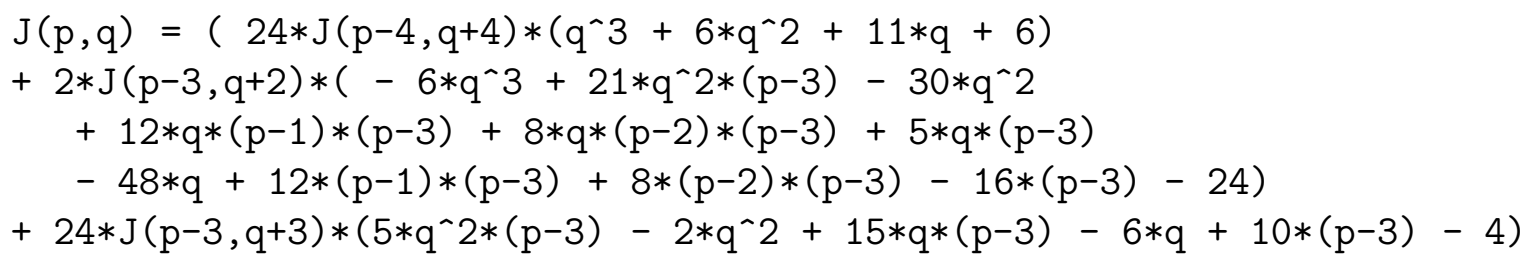




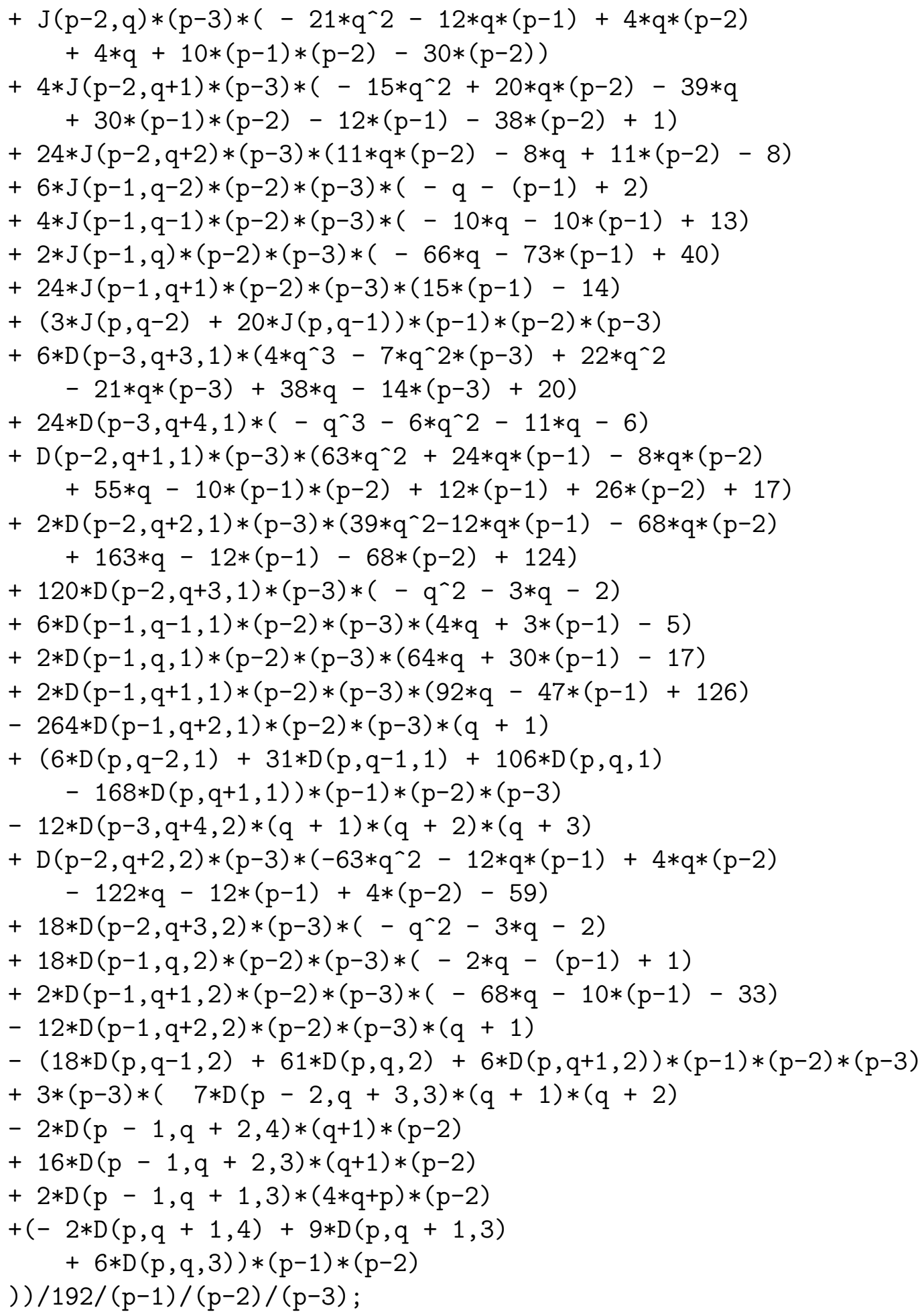

\section{Appendix 10.}

$J(4,-1)=-523385 / 2976768 Y_{11}-104677 / 91392 Y_{10}-17130665 / 26790912 Y_{9}$

$+523385 / 459648 Y_{8}+11014687 / 5515776 Y_{7}-104677 / 31256064 Y_{5}$

$-53544991 / 62512128 Y_{4}-3 Y_{0}+11316787 / 7354368(2 \pi)^{-2}-3 / 4 F_{0}(2 \pi)^{-2}$;

$J(5,-1)=881852823875 / 3876752130048 Y_{11}+176370564775 / 119023091712 Y_{10}$ 
$+28894631548079 / 34890769170432 Y_{9}-881852823875 / 598616137728 Y_{8}$

$-17115981580297 / 7183393652736 Y_{7}+176370564775 / 40705897365504 Y_{5}$

$+86132421399433 / 81411794731008 Y_{4}+25 / 8 Y_{0}$

$+\left(25 / 32 F_{0}-20532421134805 / 9577858203648\right)(2 \pi)^{-2}$;

$J(6,-1)=-28542210469686553 / 93496923371077632 Y_{11}$

$-28542210469686553 / 14352597885911040 Y_{10}-2087037111021330529 / 4207361551698493440 Y_{9}$ $+28542210469686553 / 14437024932298752 Y_{8}+2217244724616422759 / 866221495937925120 Y_{7}$ $-28542210469686553 / 4908588476981575680 Y_{5}-12694496481519981287 / 9817176953963151360 Y_{4}$ $-3163 / 960 Y_{0}+\left(3108094517023801819 / 1154961994583900160-3163 / 3840 F_{0}\right)(2 \pi)^{-2}$;

$J(7,-1)=318815426788929230845117 / 821909762068921372901376 Y_{11}$

$+318815426788929230845117 / 126170358212334421278720 Y_{10}$

+11542347686773160604861797/36985939293101461780561920 $Y_{9}$

$-318815426788929230845117 / 126912536790054035521536 Y_{8}$

$-22134243295873177135745923 / 7614752207403242131292160 Y_{7}$

$+318815426788929230845117 / 43150262508618372077322240 Y_{5}$

$+135786390066815073864737539 / 86300525017236744154644480 Y_{4}+14099 / 3840 Y_{0}$

$-34028993605943331376331719 / 10153002943204322841722880(2 \pi)^{-2}+14099 / 15360 F_{0}(2 \pi)^{-2}$;

$J(8,-1)=-9235026172226013567109728738337 / 18732046758141338739230512250880 Y_{11}$

$-9235026172226013567109728738337 / 2875533493574328315232754073600 Y_{10}$

$-55876713828271512392254716439529 / 842942104116360243265373051289600 Y_{9}$

+9235026172226013567109728738337/2892448396477706717087064391680 Y8

$+582625738707581471281711807374367 / 173546903788662403025223863500800 Y_{7}$

$-9235026172226013567109728738337 / 983432454802420283809601893171200 Y_{5}$

$-3802005063193739791001963690889631 / 1966864909604840567619203786342400 Y_{4}$

$-150949 / 35840 Y_{0}+965476530505339393064578836261427 /$

$231395871718216537366965151334400(2 \pi)^{-2}-150949 / 143360 F_{0}(2 \pi)^{-2}$;

$J(9,-1)=$

$10189310117043029375946591507272718551 / 16263612564540505685529672268508037120 Y_{11}$ $+10189310117043029375946591507272718551 / 2496607191925077627164642672797286400 Y_{10}$ $-150087551903588632763072238483785668913 / 731862565404322755848835252082861670400 Y_{9}$ $-10189310117043029375946591507272718551 / 2511293116583460436736199394401976320 Y_{8}$ $-598626882055818268392045217522764699881 / 150677586995007626204171963664118579200 Y_{7}$ $+10189310117043029375946591507272718551 / 853839659638376548490307794096671948800 Y_{5}$ $+4095937462034066852819132682453485174633 / 1707679319276753096980615588193343897600 Y_{4}$ $+8535263 / 1720320 Y_{0}+8535263 / 6881280 F_{0}(2 \pi)^{-2}$

$-1051450108870580974979233285323814292021 / 200903449326676834938895951552158105600(2 \pi)^{-2}$; $J(4,-2)=775 / 124032 Y_{11}+155 / 3808 Y_{10}+2315881 / 1116288 Y_{9}-775 / 19152 Y_{8}$ $-535775 / 229824 Y_{7}+155 / 1302336 Y_{5}+1268159 / 2604672 Y_{4}+4 Y_{0}$ 
$-256019 / 306432(2 \pi)^{-2}+F_{0}(2 \pi)^{-2}$

$J(5,-2)=-35198207035 / 161531338752 Y_{11}-7039641407 / 4959295488 Y_{10}$

$-1590044736319 / 1453782048768 Y_{9}+35198207035 / 24942339072 Y_{8}$

$+824164813817 / 299308068864 Y_{7}-7039641407 / 1696079056896 Y_{5}$

$-3786323755001 / 3392158113792 Y_{4}-4 Y_{0}+\left(886011822725 / 399077425152-F_{0}\right)(2 \pi)^{-2}$;

$J(6,-2)=11638328158211917 / 35061346264154112 Y_{11}$

$+11638328158211917 / 5382224207216640 Y_{10}+1835049614849615189 / 1577760581886935040 Y_{9}$

$-11638328158211917 / 5413884349612032 Y_{8}-1118098215139974931 / 324833060976721920 Y_{7}$

$+11638328158211917 / 1840720678868090880 Y_{5}+5661105033256044691 / 3681441357736181760 Y_{4}$

$+181 / 40 Y_{0}+\left(181 / 160 F_{0}-1412418713096881687 / 433110747968962560\right)(2 \pi)^{-2}$;

$J(7,-2)=-16095494538985786829003 / 34246240086205057204224 Y_{11}$

$-16095494538985786829003 / 5257098258847267553280 Y_{10}$

$-1220878670422477200084451 / 1541080803879227574190080 Y_{9}$

$+16095494538985786829003 / 5288022366252251480064 Y_{8}$

$+1258484886934090324074869 / 317281341975135088803840 Y_{7}$

$-16095494538985786829003 / 1797927604525765503221760 Y_{5}$

$-1435246543741057501042993 / 719171041810306201288704 Y_{4}-2447 / 480 Y_{0}$

$+\left(1817967522495901075073489 / 423041789300180118405120-2447 / 1920 F_{0}\right)(2 \pi)^{-2}$;

$J(8,-2)=97496623093573646692443907729 / 156100389651177822826920935424 Y_{11}$

+97496623093573646692443907729/23962779113119402626939617280 Y 10

$+703823889446906512753183459525 / 1404903506860600405442288418816 Y_{9}$

-97496623093573646692443907729/24103736637314222642392203264 $Y_{8}$

-6768519700158561511510642407343/1446224198238853358543532195840 Y

+97496623093573646692443907729/8195270456686835698413349109760 Y

$+41525287801127597648514834574639 / 16390540913373671396826698219520 Y_{4}$

$+79489 / 13440 Y_{0}+79489 / 53760 F_{0}(2 \pi)^{-2}$

$-2132153450344177531160317231847 / 385659786197027562278275252224(2 \pi)^{-2}$;

$J(9,-2)=-184720162027043790012174961950856949 / 225883507840840356743467670395944960 Y_{11}$

$-184720162027043790012174961950856949 / 34675099887848300377286703788851200 Y_{10}$

-1182390536568106894749732117915566173/10164757852837816053456045167817523200 Y9

+184720162027043790012174961950856949/34879071063659172732447213811138560 Y

$+11666341875097592955381754264025444939 / 2092744263819550363946832828668313600 Y_{7}$

-184720162027043790012174961950856949/11858884161644118729032052695787110400 $Y_{5}$

$-76075734513749258577040135726310510027 / 23717768323288237458064105391574220800 Y_{4}$

$-501267 / 71680 Y_{0}-501267 / 286720 F_{0}(2 \pi)^{-2}$

$+19693107617724772864308858550443517679 / 2790325685092733818595777104891084800(2 \pi)^{-2}$; 


\section{Appendix 11.}

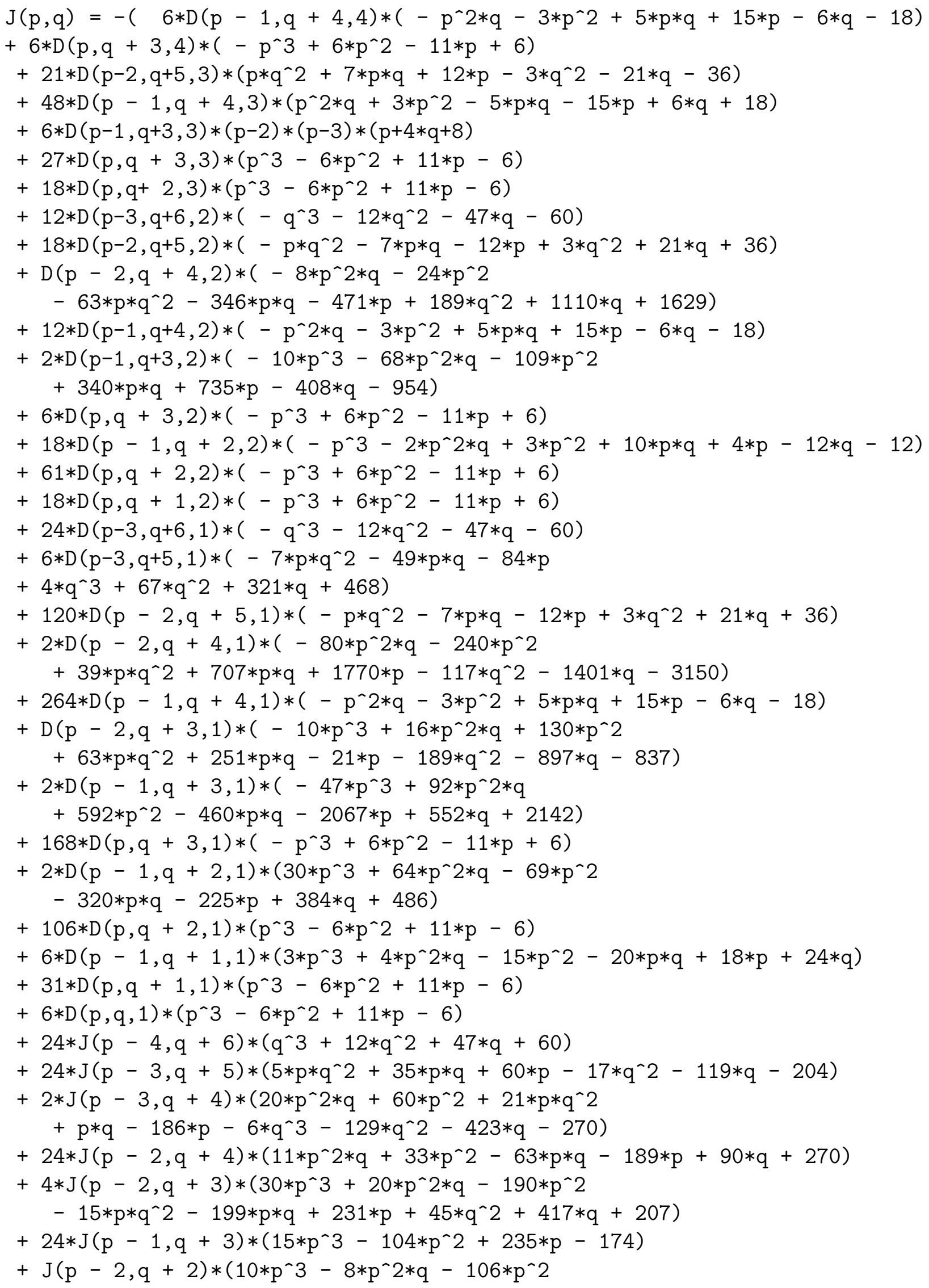




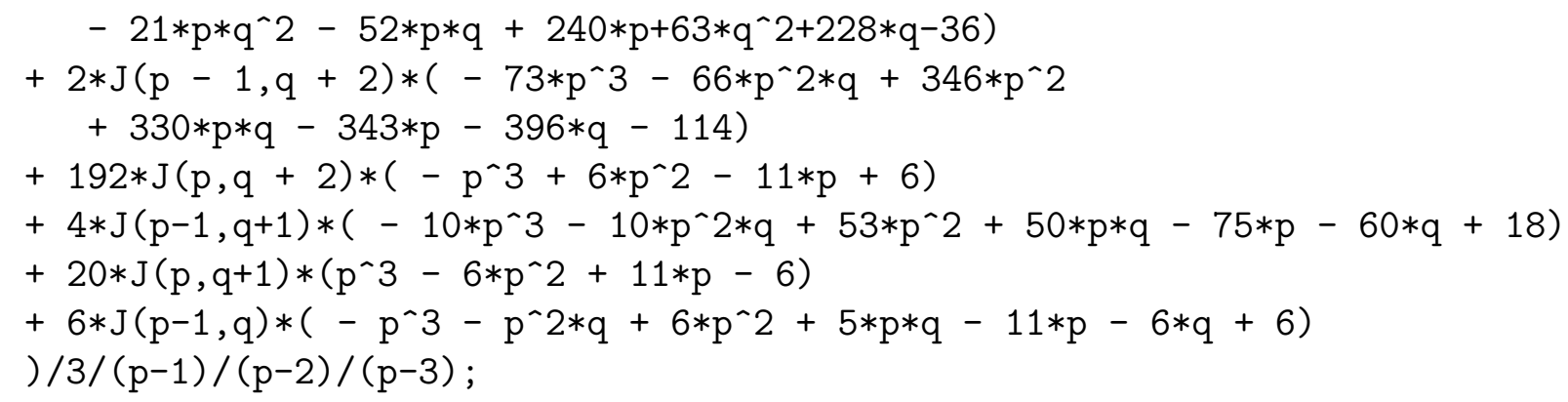

\section{References}

[1] S. Capitani, Phys. Rep., v. 382 (2003) p. 113.

[2] H. Kawai, R. Nakayama, and K.Seo Nucl. Phys. B189 (1981) p.40.

[3] S. Capitani et al., Nucl.Phys., B593 (2001) p.183.

[4] M. Gockeler et al., Eur.Phys.J. C48 (2006) p.523; Erratum: Eur.Phys.J. C56 (2006) p.161.

[5] G. Burgio, S. Caracciolo, A. Pelissetto, Nucl.Phys., B478 (1996), pp. 687-722.

[6] T. Becher and K. Melnikov, Phys.Rev., D66:074508 (2002).

[7] J.A.M.Vermaseren, "New features of FORM", math-ph/0010025

[8] A.C. Hearn, REDUCE user's manual, Version 3.6. Report CP 78, RAND, July 1995. 